\title{
SIVARAMAKRISHNA ANANTHARAMAN Schémas en groupes, espaces homogènes et espaces algébriques sur une base de dimension 1
}

\author{
Mémoires de la S. M. F., tome 33 (1973), p. 5-79 \\ <http://www.numdam.org/item?id=MSMF_1973_33_5_0>
}

CC Mémoires de la S. M. F., 1973, tous droits réservés.

L'accès aux archives de la revue « Mémoires de la S. M. F. » (http://smf. emath.fr/Publications/Memoires/Presentation.html) implique l'accord avec les conditions générales d'utilisation (http://www.numdam.org/conditions). Toute utilisation commerciale ou impression systématique est constitutive d'une infraction pénale. Toute copie ou impression de ce fichier doit contenir la présente mention de copyright.

\section{Numdam}


Bull. Soc. math. France,

Mémoire 33, 1973, p. 5-79.

SCHEMAS EN GROUPES, ESPACES HOMOGENES ET ESPACES ALGÉBRIZUES

SUR UNE BASE DE DIMENSION 1

par

Sivaramakrishna ANANTHARAMAN

$-:-:-:-$

Résumé. - Soient $S$ un schéma localement noethérien, et $X$ un S-espace algébrique en groupes séparé et localement de type fini sur $S$ (resp. le $S$-faisceau fppf quotient d'un S-schéma en groupes G, localement de type fint, par un sous-schéma fermé en groupes $H$, plat sur $S$ ). Lorsque $S$ est de dimension 0 , on sait que $X$ est représentable. Par contre, même dans les meilleurs cas, un tel $X$ 'est pas nécessairement représentable si dim $S \geqslant 2$. Dans ce travail, on démontre la représentabilité de tels $X$ lorsque dim $S \leqslant 1$; si $S$ est normal, la démonstration utilise des techniques (à peu près connues) spéciales au cas dim $S=1$; le cas général se résoud ensuite moyennant un critère (général) de descente d'espaces algébriques par des morphismes entiers.

\section{Table des matieres}

Pages

Introduction.$\ldots \ldots \ldots \ldots \ldots \ldots \ldots \ldots \ldots \ldots \ldots \ldots \ldots \ldots \ldots \ldots \ldots \ldots \ldots \ldots \ldots \ldots \ldots$

Chapitre I. - Le recollement suivant un fermé. Applications. ........... 8

1.1 Le recollement suivant un fermé. ........................ 10

1.2 Application aux questions de descente fpqc . .............. 15

1.3 Application aux espaces algébriques. .................... 19

Chapitre II. - Schémas en groupes sur un schéma de Dedekind. ........... 22

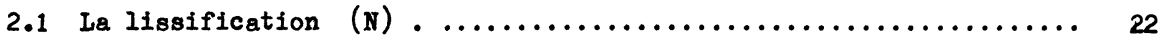

2.2 Une application aux groupes commutatifs. ................... 25

2.3 Schémas en groupes et morphismes affines. .................... 30

Chapitre III. - Sur les faisceaux quotients et les anneaux algébriques. .... 35

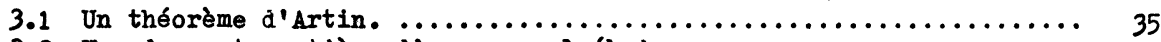

3.2 Une descente entiere d'espaces algébriques. ................. 40

3.3 Eristence du quotient en codimension $1 . \ldots \ldots \ldots \ldots \ldots \ldots \ldots \ldots \ldots . . \ldots 49$

3.4 Application aux espaces homogenes. ..................... 52

Chapitre IV. - Applications aux questions de représentabilité. .......... 53

Appendice I. - Passage au quotient par un grouporde plat. ............. 63

A. Réduction au cas d'un grouporde quasi-fini. ............... 63

B. Un critere de passage au quotient. ....................... 66

Appendice II. - Normalisé d'un schéma sur un schéma de Dedekind. ......... 73

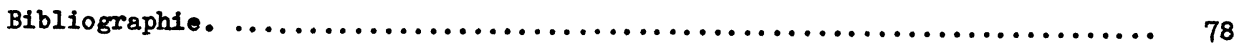




\section{Introduction}

Depuis que la notion d'espace algébrique a été introduite en Géométrie algébrique par M. Artin, de nombreuses questions de représentabilité ont été traitées suivant deux principes généraux.

(a) Etudier les conditions pour qu'un foncteur soit un espace algébrique.

(b) Etudier les conditions pour qu'un espace algébrique soit un schéma.

Dans le présent travail, on étudie certains cas particuliers de (b). On sait par exemple que, sur une base de dimension zéro, un espace algébrique en groupes $G$ est un schéma ([2], §4). Sur un corps $k$, ce fait se démontre de la façon suivante : il existe un ouvert dense $X$ de $G$ qui est un schéma ( $S G A D, V, 8.1$ ); et sur la clôture algébrique $\bar{k}$ de $k$, on dispose de suffisamment de sections de $G$ pour pouvoir recouvrir $G$ par les translatés de cet ouvert $X$. La descente de $\bar{k}$ à $k$ est possible, comme chaque ensemble fini de points de $G_{\bar{k}}$ est contenu dans un ouvert affine.

On peut essayer d'appliquer une telle méthode sur une base $S$ quelconque. Mais, pour ce faire, on doit a priori disposer d'un ouvert représentable $X$ de $G$ tel que $X$ soit dense dans chacune des fibres de $G$ sur $S$. Or, en général, il n'existe pas de tel $X$ : en effet, on a un espace algébrique en groupes $G$ lisse et non séparé sur un trait, dont le plus grand ouvert représentable est sa fibre générique ( $\mathrm{SGAD}, \mathrm{VI}_{B}$, page 81 ). Même lorsque l'on fait l'hypothèse de séparation, il existe un contre-exemple de Raynaud, sur une base locale régulière de dimension 2 ([18], X 14).

En revanche, on peut montrer, avec l'hypothèse de séparation, que le plus grand ouvert représentable $X$ d'un espace algébrique $G$ contient ses points de codimension 1 (cf. (3.3.1)); si $S$ est de dimension $\leqslant 1$, cet ouvert $X$ contient donc les points maximaux des fibres de $G$ sur $S$.

Nous étudions par suite les questions "maturelles" suivantes :

$1^{\circ}$ Soient $S$ un schéma localement noethérien de dimension $\leqslant 1$, G un $S-$ espace algébrique en groupes, séparé et localement de type fini sur $S$. Alors $G$ est-il un schéma?

$2^{\circ}$ Soient $S$ comme dans le $1^{\circ}, G$ un S-faisceau fppf en groupes, $S^{\prime}$ un schéma fpqc sur $S$ tel que $G_{S^{\prime}}$ soit un $S^{\prime}-s c h e ́ m a$ (en groupes) séparé et localement de présentation finie. Alors $G$ est-il un schéma?

$3^{\circ}$ Nous étudions également la question d'existence du schéma quotient du type $\mathrm{G} / \mathrm{H}$. On sait que les faisceaux de ce type sont des espaces algébriques sur une base 
S quelconque localement noethérienne (cf. (3.1.1)). Par contre, la question de représentabilité de ces faisceaux admet une réponse négative si l'on ne suppose pas $H$ fermé dans $G$ et plat sur $S$, ou si dim $S \geqslant 2$ (mêmes contre-exemples que plus haut). Nous étudions donc le cas où $S$ est localement noethérien de dimension $\leqslant 1$.

A ces trois questions, nous répondons affirmativement; nous montrons aussi que chaque ensemble fini de points de G (resp. G/H) qui se projette dans un ouvert affine de $S$ est contenu dans un ouvert affine de $G$ (resp. G/H ).

Esquissons brièvement la méthode de démonstration. On note d'abord que les fibres sont des schémas. Sur une base normale, par exemple sur un trait, la notion d'adhérence schématique de la fibre générique de $G$ (cf. (1.2.0)) et une technique du recollement suivant un fermé (cf. (1.1)) nous permettent de supposer que $G$ est plat sur S (cf. (1.2) et (1.3)). En utilisant l'appendice II, on se ramène alors au cas G lisse sur $S$ (chap. IV). On applique ensuite les résultats de la thèse de Raynaud $([18])$.

Dans le cas où $S$ est non normal, on utilise un critère général de descente entière (cf. (3.2.1)) qui ne dépend pas de la structure de groupe sur $G$ ou de la structure homogène sur G/H .

Faute de références satisfaisantes, nous avons da démontrer en détail certains résultats "connus", comme le recollement suivant un fermé, ou le passage au quotient plat (resp. fini).

L'auteur tient à remercier vivement M. RAYNAUD, dont l'encouragement et les conseils lui ont été une aide précieuse pour ce travail. 
Chapitre I

Recollement suivant un fermé. Applications

1.0. Un trait est par définition un triple $(s=\operatorname{spec} \Lambda, \eta, s)$ où $\Lambda$ est un anneau de valuation discrète et $\eta$ (resp. s) est le point générique (resp. fermé) de $S=\operatorname{sped} \Lambda$

Süposons que $\mathrm{X}=\operatorname{Spec} \mathrm{A}$ est un schéma affine noethérien sur un trait ( $\operatorname{spec} \Lambda=S, \eta, s)$. Soient $\pi$ une uniformisante de $\Lambda$ et $T$ l'idéal de $\Lambda$-torsion dans A. Alors on a la notion d'adhérence schématique $\bar{X}$ de la fibre générique $X_{\eta}$ de $X$ dans $X$ : c'est le sous-schéma fermé $V(T)$ de $X$. C'est aussi le plus grand sous-schéma de $X$ plat sur $S$. D'autre part, on a également les voisinages infinitésimaux $\left\{x_{s}^{(n)}\right\}_{n \geqslant 0}$ de la fibre spéciale $X_{s}$ : ce sont les sous-schémas fermés $\left\{V\left(\pi^{n} A\right)\right\}_{n \geqslant 0}$ de $X$.

Il $\mathrm{y}$ a une façon naturelle de reconstituer $\mathrm{X}$ à partir de la donnée de $\overline{\mathrm{X}}$ et $\operatorname{des}\left\{\mathrm{X}_{\mathrm{s}}^{(\mathrm{n})}\right\}_{\mathrm{n} \geqslant 0} ;$ topologiquement c'est très clair ; il s'agit d'identifier les points communs de $\bar{X}$ et $X_{S}$. Puisque ces points communs forment une partie fermée $\bar{X} \cap X_{S}$ de $X$, il s'agit de "recoller" $\bar{X}$ et $X_{s}$ suivant ce fermé commun.

Pour tenir compte du faisceau structural, nous procédons de la manière suivante. Pour chaque $n \geqslant 0$, considérons "l'intersection schématique" de $\bar{X}$ et $x_{s}^{(n)}$ : c'est le sous-schéma fermé $\bar{X} \times X_{S}{ }_{s}^{(n)}=V\left(\pi^{n} A+T\right)$ de $X$, donc c'est un sous-schéma fermé à la fois de $\bar{X}$ et de $x_{s}^{(n)}$. On a ainsi deux plongements

$$
V\left(\pi^{n} A+T\right) \rightarrow \bar{X} \Perp x_{s}^{(n)}
$$

Ces deux plongements correspondent aux deux morphismes composés

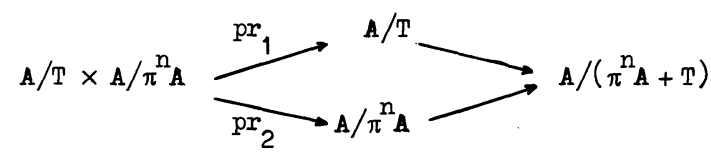

(où les flèches sont évidentes). 
Si $B^{(n)}$ est le sous-anneau (de $A / T \times A / \pi^{n} A$ ) d'égalité de ces deux flèches, il se trouve que $\operatorname{Spec} B^{(n)}$ est le conoyau de la double flèche

$$
V\left(\pi^{n} A+T\right) \rightarrow x \Perp x_{s}^{(n)}
$$

dans la catégorie $(\mathrm{Sch} / \mathrm{s})$ (voir (1.1.3) ci-dessous). On a alors un morphisme canonique $\varphi^{(n)}: \operatorname{Spec} B^{(n)} \rightarrow X$. Je dis que pour tout $n$ assez grand $\varphi^{(n)}$ est un isomorphisme.

En effet, il est clair que $\varphi^{(n)}$ correspond au morphisme canonique
$\mathrm{A} \stackrel{\theta^{(n)}}{\longrightarrow} \mathrm{A} / \mathrm{T} \times \mathrm{A} / \pi^{\mathrm{n}} \mathrm{A}$ qui se factorise par $\mathrm{B}^{(n)}$. D'autre part, si $a_{1}, a_{2} \in \mathrm{A}$ sont tels que $a_{1} \equiv a_{2}\left(\pi^{n} A+T\right)$ alors $a_{1}=a_{2}+\pi^{n} a^{\prime}+t^{\prime}$ avec $a^{\prime} \in A$, t' $\in T$, d'où $\left(a_{1}-\pi^{n} a^{\prime}\right)=\left(a_{2}+t^{\prime}\right)$ est un élément a de $A$ tel que $a \equiv a_{1}\left(\pi^{n} A\right)$ et $a \equiv a_{2}(T)$. Ce qui prouve que $\theta^{(n)}(A)=B^{(n)}$, donc $\varphi^{(n)}$ est une immersion fermée pour tout $n$. Enfin le noyau de $\theta^{(n)}$ est $\left(\pi^{n} A\right) \cap T=\pi^{n} T$ et comme $T$ est engendré par un nombre fini d'éléments (A noethérien), $\pi^{\mathrm{n}} \cdot \mathrm{T}=0$ pour $\mathrm{n}$ assez grand. Donc $\theta^{(n)}: A \stackrel{A}{\sim} B^{(n)}$ pour $n$ assez grand。 Tout cela prouve que $X$ est le schéma obtenu par "recollement" de $\bar{X}$ et $x_{s}^{(n)}$ suivant le fermé commun $\overline{\mathrm{X}} \times{ }_{\mathrm{X}} \mathrm{X}_{\mathrm{S}}^{(\mathrm{n})}$ pour $\mathrm{n}$ assez grand.

Il se trouve que plusieurs aspects de ce phénomène se prêtent à des généralisations. Ainsi, pour un foncteur quelconque $G$ de $(\mathrm{Sch} / \mathrm{S})^{\cdot}$ dans $\mathrm{Ens}$, on a la notion d'adhérence schématique $\bar{G}$ de $G_{\eta}$ dans $G$ (s étant toujours un trait; voir $(1.2 .0)$ ci-dessous), et sous certaines conditions, si $\bar{G}$ et les $G_{S}^{(n)}$ sont des schémas, on peut représenter G "en recollant $\bar{G}$ et $G_{S}^{(n)}$ suivant un fermé" (voir $(1.2 .4)$ et $(1.3 .2)$ ci-dessous). L'avantage de cette méthode est qu'elle ramène la question de la représentabilité de $G$ sur $S$ à celle des $G_{S}^{(n)}$ (qui sont des foncteurs sur un schéma artinien) et celle de $\bar{G}$, qui est alors un foncteur "plat sur $S "$ se prêtant donc à plus de manipulations que $G$. 


\subsection{Le recollement suivant un fermé}

1.1.0. On se pose la question suivante. Etant donnés trois schémas $X_{1}, X_{2}, Y$ et deux immersions fermées $Y \stackrel{\theta_{i}}{\longrightarrow} X_{i}, i=1,2$, peut-on construire un schéma $X$ en recollant $X_{1}, X_{2}$ suivant $\left(Y, \theta_{1}, \theta_{2}\right)$ ?

Plus précisément, la donnée $\left(Y, \theta_{1}, \theta_{2}\right)$ définit une relation d'équivalence évidente sur $\mathrm{X}_{1} \Perp \mathrm{X}_{2}$ :

$$
Y \underset{\theta_{2}}{\stackrel{\theta_{1}}{\longrightarrow}} X_{1} \Perp X_{2} .
$$

Soient $X=X_{1} \frac{\|}{\theta} X_{2}=X_{1} \frac{H}{Y} X_{2}$ (où $\theta=\left(\theta_{1}, \theta_{2}\right)$ ) l'espace annelé quotient de $\mathrm{X}_{1} \Perp \mathrm{X}_{2}$ pour cette relation et $\varphi_{i}: \mathrm{X}_{i} \rightarrow \mathrm{X}$ les morphismes canoniques d'espaces annelés. On a alors la

1.1.1. Proposition : $X=X_{1} \frac{\| 1}{\theta} X_{2}$ est un schéma et les $\varphi_{i}$ sont des immersions fermées dans (Sch). Par suite il existe un sous-schéma fermé $\bar{Y}$ de $X$, à espace sous-jacent $\varphi_{1}\left(x_{1}\right) \cap \varphi_{2}\left(X_{2}\right)$ tel que $\varphi_{i} \cdot \theta_{i}: Y_{i} \rightarrow \bar{Y}, i=1,2$, soient des isomorphismes.

\subsubsection{Remarques :}

(a) Supposons que, pour chaque $i, X_{i} \hookrightarrow X_{i}$ soit un ouvert tel que $\theta_{1}^{-1}\left(X_{1}^{\prime}\right)=\theta_{2}^{-1}\left(X_{2}^{\prime}\right)=Y^{\prime}$. Alors $\theta_{i}^{\prime}=\theta_{i} \mid Y^{\prime}: Y^{\prime} \rightarrow X_{i}^{\prime}$ sont des immersions fermées, et il est clair que $X_{1}^{\prime} \frac{\|}{\theta^{\prime}} X_{2}^{\prime}$ (où $\theta^{\prime}=\left(\theta_{1}^{\prime}, \theta_{2}^{\prime}\right)$ ) s'identifie canoniquement à un ouvert de $x_{1} \frac{\|}{\theta} x_{2}$ 。

(b) Supposons démontrée la proposition $(1 \cdot 1 \cdot 1)$. Il est alors immédiat que le schéma $\mathrm{X}_{1} \frac{\Perp}{\theta} \mathrm{X}_{2}$ est le quotient de $\mathrm{X}_{1} \Perp \mathrm{X}_{2}$ pour la relation d'équivalence définie par $\left(\theta_{1}, \theta_{2}\right)$ dans le catégorie ( $\mathrm{Sch}$ ).

(c) Soient $S$ un schéma et $Z$ un schéma sur $S$. Nous dirons que $Z$ est de type (FA) sur $S$ si chaque ensemble fini de points de $Z$ qui se projette dans un ouvert affine de $S$, est lui-même contenu dans un ouvert affine de $\mathbf{Z}$. Un schéma $Z$ est dit de type (FA) s'il est de type (FA) sur Spec $Z$. Revenant à la situation précédente, nous démontrerons que si chaque $X_{i}$ est de type (FA), il en est de même de $X=X_{1} \frac{\|}{\theta} X_{2}$. En fait nous montrerons un peu plus: 
Si $E$ est un ensemble fini de points de $X$ tel que chaque $E_{i}=\varphi_{i}^{-1}(E)$ soit contenu dans un ouvert affine de $X_{i}$, alors $E$ est contenu dans un ouvert affine de $X$

\subsubsection{Démonstration de 1.1 .1 dans le cas affine :}

Supposons que $X_{i}=\operatorname{Spec}\left(A_{i}\right), i=1,2, Y=\operatorname{Spec}(\bar{A})$ et $Y_{i}=\theta_{i}(Y)=V\left(I_{i}\right)$ où $I_{i}$ est un idéal de $A_{i}, i=1,2$. On a alors un diagramme

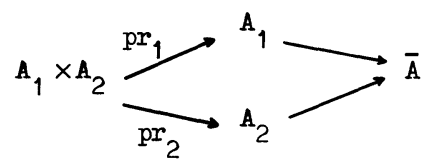

où les morphismes sont évidents. Posons $B=l e$ sous-anneau ( de $\left.A_{1} \times A_{2}\right)$ d'égalité des deux flèches composées $A_{1} \times A_{2} \rightarrow \vec{A}$. Je dis que $X=$ Spec $B$ s'identifie alors à l'espace annelé $X_{1} \frac{1}{\theta} X_{2}$.

i) Puisque $\bar{A}$ est un quotient de $A_{i}, p_{i}=p_{i} \mid B$ est surjectif ce qui nous donne des immersions fermées $\varphi_{i}: X_{i} \hookrightarrow X \cdot D^{\prime}$ autre part, si $K_{i}=\operatorname{ker}\left(B \stackrel{p_{i}}{\rightarrow} A_{i}\right)$, il est clair que $K_{1} \simeq(0) \times I_{2}, K_{2} \simeq I_{1} \times(0)$, donc $K_{1} \cdot K_{2}=0$ dans $B$. Ce qui montre que : $X=\varphi_{1}\left(X_{1}\right) \cup \varphi_{2}\left(X_{2}\right)$ ensemblistement.

ii) Or, $X-\varphi_{2}\left(X_{2}\right)=\bigcup_{g \in K_{2}} \operatorname{Spec} B_{g}$; un élément $g \in K_{2}$ est de la forme $(f, 0), f \in I_{1}$ et réciproquement; d'autre part, il est clair que $\varphi_{1}^{-1}\left(\right.$ Spec $\left.B_{g}\right)$ est $I^{\prime}$ ouvert affine $\operatorname{Spec}\left(A_{1}\right)_{f}$ de $X_{1}$. Comme $X_{1}-Y_{1}=\underset{f \in I_{1}}{U} \operatorname{Spec}\left(A_{1}\right)_{f}=\underset{g \in K_{2}}{U} \varphi_{1}^{-1}\left(\operatorname{Spec} B_{g}\right)$ et que $B_{g} \cong\left(A_{1}\right)_{f}$ pour $f \in I_{1}$ et $g=(f, 0) \in K_{2}$, on a montré que :

$\varphi_{1} \mid\left(X_{1}-Y_{1}\right)$ est un isomorphisme sur l'ouvert $X-\varphi_{2}\left(X_{2}\right)$ de $X$ (on a aussi I'asm sertion analogue pour $\left.\varphi_{2} \mid \mathrm{X}_{2}-\mathrm{Y}_{2}\right)$.

iii) Enfin, soit $J=\operatorname{ker}(B \rightarrow \bar{A})$ où la flèche est le morphisme unique provenant de la définition de $B$; il est clair que $\left(a_{1}, a_{2}\right) \in J$ si et seulement si $a_{i} \in I_{i}, i=1,2$, d'où $J=K_{1}+K_{2}$ dans $B$. Et puisque $B / J \cong \vec{A}$, on en conclut que $\mathrm{V}(\mathrm{J})$ est égal à $\varphi_{1}\left(\mathrm{x}_{1}\right) \cap \varphi_{2}\left(\mathrm{x}_{2}\right)$, schématiquement.

On déduit de $i)$, $i i)$ et $i i i)$ que $\left(X=\operatorname{spec} B, \varphi_{1}, \varphi_{2}\right)$ est bien I'espace annelé quotient de $\mathrm{X}_{1} \Perp \mathrm{X}_{2}$ pour la relation d'équivalence spec $\overline{\mathrm{A}} \rightarrow \mathrm{X}_{1} \Perp \mathrm{X}_{2}$. 
1.1.4. Démonstration de 1.1 .1 dans le cas général :

Le fait que l'espace annelé $\mathrm{X}=\mathrm{X}_{1} \frac{\|}{\theta} \mathrm{X}_{2}$ soit un schéma se vérifie localement sur X ; donc, grâce à 1.1.2 (a) et 1.1.3 il suffit de montrer la chose suivante :

Soit $y$ un point quelconque de $Y, y_{i}=\theta_{i}(y)$. Alors il existe un ouvert affine $U_{1}$ de $x_{1} \quad\left(r e s p . U_{2}\right.$ de $\left.x_{2}\right)$ contenant $y_{1} \quad\left(\right.$ resp. $\left.y_{2}\right)$ tel que $\theta_{1}^{-1}\left(\sigma_{1}\right)=\theta_{2}^{-1}\left(\sigma_{2}\right)$.

Mais afin de démontrer du même coup la remarque 1.1 .2 (c), nous allons prouver un peu plus :

(*) Soit $E$ un ensemble fini de points de $X$ tel que $E_{i}=\varphi_{i}^{-1}(E)$ soit contenu dans un ouvert affine de $x_{i}, i=1,2$. Alors il existe un ouvert affine $v_{1}$ de $x_{1}$ (resp. $v_{2}$ de $x_{2}$ ) contenant $E_{1}$ (resp. $E_{2}$ ) tel que $\theta_{1}^{-1}\left(v_{1}\right)=\theta_{2}^{-1}\left(v_{2}\right)$. Commençons.par un lemme facile.

Lemme(F): Soit $F$ un ensemble fini de points d'un schéma affine $Z=$ Spec A . Si $U$ est un ouvert quelconque de $Z$ contenant $F$ alors il existe un a $\in A$ tel que $\mathrm{F} \subset \mathrm{z}_{\mathrm{a}} \subset \mathrm{U}$.

En effet, si $p_{x}$ est l'idéal premier de $\mathbf{A}$ défini par un point quelconque $\mathbf{x}$ de $Z$ et si $I$ est un idéal de définition de $z-U$ dans $A$, on a $p_{x} \not \supset I$ pour $x \in F$. Comme $F$ est fini, on a $I \not \subset \bigcup_{x \in F} p_{x}$ et $i l$ existe un a $\in I$ tel que a $\notin \mathrm{p}_{\mathrm{x}}$ pour tout $\mathrm{x} \in \mathrm{F}$.

Revenons à la démonstration de $(*)$. Posons $Y_{i}=\theta_{i}(Y), i=1,2$. Soit $U_{i}^{\prime}$ un ouvert affine de $x_{1}$ contenant $E_{1} ;$ alors il existe un ouvert $\sigma_{2}^{\prime}$ de $X_{2}$ tel que $U_{1}^{\prime} \cap Y_{1} \underset{\vec{\theta}}{\overrightarrow{U_{2}}} \cap Y_{2}$. Ajoutant au besoin un ouvert de $x_{2}$ contenant $E_{2}-\left(\bar{E}=\left(\varphi_{1}\left(X_{1}\right) \cap \varphi_{2}\left(X_{2}\right) \cap E\right)\right)$ et contenu dans $X_{2}-Y_{2}$, on peut supposer que $\mathrm{U}_{2}^{\prime} \supset \mathrm{E}_{2} \cdot$

Si $U_{2}^{\prime \prime}$ est un ouvert affine de $\mathrm{X}_{2}$ contenant $\mathrm{E}_{2}$, alors il existe un ouvert affine $U_{2}^{\prime \prime \prime}$ de $U_{2}^{\prime \prime}\left(\right.$ donc de $x_{2}$ ) tel que $E_{2} \subset U_{2}^{\prime \prime \prime} \subset U_{2}^{\prime \prime} \cap U_{2}^{\prime}$ (lemme(F)). Quitte à restreindre $U_{1}^{\prime}$ on est ainsi ramené à supposer que :

il existe un ouvert affine $\tilde{U}_{1}$ de $X_{1}$, un ouvert $\tilde{U}_{1}$ de $\tilde{U}_{1}$ et un ouvert 
affine $\mathrm{U}_{2}$ de $\mathrm{X}_{2}$ tels que :

a) $U_{1} \supset E_{1}, U_{2} \supset E_{2}$

b) $U_{1} \cap Y_{1} \underset{\dot{\theta}}{\sim} U_{2} \cap Y_{2}$

Soit $f \in A\left(\tilde{U}_{1}\right)$ (notation évidente) un élément tel que $E_{1} \subset \tilde{U}_{1_{f}} \subset U_{1}$ (lemme $(F)$ ); soient $\bar{f}_{1}$ son image dans $A\left(U_{1} \cap Y_{1}\right)$ et $\bar{f}_{2}$ la $\theta$ coimage de $\bar{f}_{1}$ dans $A\left(U_{2} \cap Y_{2}\right)$. Il est immédiat que tout revient à trouver un relèvement $f_{2}$ de $\bar{f}_{2}$ dans $A\left(U_{2}\right)$ tel que $U_{2_{f_{2}}} \supset E_{2}$, car on peut alors prendre $V_{1}=\tilde{U}_{1_{f_{1}}}$ et $\mathrm{v}_{2}=\mathrm{J}_{\mathrm{f}_{2}}$. (Notons d'ailleurs que dans le cas où $\mathrm{E}=\overline{\mathrm{E}}$, i.e. où $E \subset \varphi_{1}\left(x_{1}^{2}\right) \cap \varphi_{2}\left(x_{2}\right)$, tout relèvement $f_{2}$ de $\bar{f}_{2}$ dans $A\left(U_{2}\right)$ possède cette propriété; la démonstration proprement dite de 1.1 .1 s'achève donc ici).

Pour établir (*), on peut évidemment supposer que :

(**) aucun des points de $\mathrm{E}_{2} \mathrm{n}^{\prime}$ est une spécialisation stricte d'un autre point de $\mathrm{E}_{2}$ •

or, soit $I$ un idéal de définition de $U_{2} \cap Y_{2}$ dans $A\left(U_{2}\right)$ et $f_{2}^{\prime}$ un relèvement quelconque de $\bar{f}_{2}$ dans $A\left(U_{2}\right)$. Posons $E_{2}^{\prime}=\left(E_{2}-\bar{E}\right) \cap U_{2}$, et $E_{2}^{\prime \prime}=\left(E_{2}-\bar{E}\right) \cap V\left(f_{2}^{\prime}\right)$. Il est clair que I $\not p_{x}$ pour $x \in E_{2}^{\prime \prime} ;_{2}^{\prime}$ et à cause de $(* *)$ on a $p_{y} \not p_{x}$ si $y \in E_{2}^{\prime}$ et $x \in E_{2}^{\prime \prime}$. D'où I $\cap\left(\underset{y \in E_{2}^{\prime}}{n} p_{y}\right) \not \underbrace{u}_{x \in E_{2}^{\prime \prime}} p_{x} ;$ il existe donc un $g \in I \cap\left(\bigcap_{\mathrm{y} \in \mathrm{E}_{2}^{\prime}}^{n} \mathrm{p}_{\mathrm{y}}\right)$ tel que $\mathrm{g} \notin \mathrm{p}_{\mathrm{x}}$ pour $\mathrm{x} \in \mathrm{E}_{2}^{\prime \prime}$. Il est alors immédiat que $f_{2}=f_{2}^{\prime}+g$ est un relèvement de $\bar{f}_{2}$ qui répond à la question. C.Q.F.D.

\subsubsection{Remarques :}

i) Plaçons-nous désormais sur un schéma de base $\mathrm{S}$. Si $\left(X_{1}, X_{2}, Y, \theta_{1}, \theta_{2}\right)$ est une donnée de recollement du type 1.1.0 dans (Sch/s) alors $x=x_{1} \frac{\| l}{\theta} x_{2}$ est évidemment dans $(s c h / s)$. De plus il est facile à voir que si chaque $X_{i}$ est S-séparé, $X$ l'est aussi.

Suppósons que $S^{\prime} \in(\mathrm{Sch} / \mathrm{S})$. Alors, on obtient par le changement de base $S^{\prime} \rightarrow S$, une donnée de recollement $\left(X_{1}^{\prime}=X_{1_{S^{\prime}}}, X_{2}^{\prime}=X_{{ }_{S^{\prime}}}, Y^{\prime}=Y_{S^{\prime}}, \theta_{1}^{\prime}=\theta_{1_{S^{\prime}}}\right.$, $\left.\theta_{2}^{\prime}=\theta_{2}{ }_{S^{\prime}}\right)$ dans (Sch/s'). Ce qui nous permet de construire le $S^{\prime}$-schéma $\mathrm{X}_{1}^{\prime} \frac{\| l}{\theta^{\prime}} \mathrm{X}_{2}^{\prime}$ (où $\theta^{\prime}=\left(\theta_{1}^{\prime}, \theta_{2}^{\prime}\right)$ ). $D^{\prime}$ après $1.1 .2(\mathrm{~b})$, il existe un $\mathrm{S}^{\prime}$-isomorphisme 
canonique $\mathrm{X}_{1}^{\prime} \frac{\|}{\theta^{\prime}} \mathrm{X}_{2}^{\prime} \rightarrow\left(\mathrm{x}_{1} \frac{\|}{\theta} \mathrm{X}_{2}\right)_{\mathrm{S}^{\prime}}$.

La démonstration de 1.1 .1 montre que si $S^{\prime}$ est plat sur $S$, alors c'est un isomorphisme. En d'autres termes, le recollement suivant un fermé commute aux changements de base plats.

ii) Supposons que $S$ est localement noethérien et que $\left(X_{1}, X_{2}, Y, \theta_{1}, \theta_{2}\right)$ est une donnée du type 1.1.0 localement de type fini sur $S$ (i.e., chaque $X_{i} l^{\prime}$ est). Alors une démonstration analogue à celle du théorème 5 de I'appendice I montre que $\mathrm{X}_{1} \frac{\|}{\theta} \mathrm{X}_{2}$ est aussi localement de type fini sur $\mathrm{S}$. iii) Soient $s$ localement noethérien, $\left(X_{1}, X_{2}, Y, \theta_{1}, \theta_{2}\right)$ une donnée $d u$ type 1.1.0 localement de type fini sur $S$ et $\left(X_{1}^{\prime}, X_{2}^{\prime}, Y^{\prime}, \theta_{1}^{\prime}, \theta_{2}^{*}\right)$ une deuxième donnée étale sur la première ( $i_{\bullet} e_{\bullet}$, on a des $S$-morphismes étales tels que tous les carrés évidents soient cartésiens). Alors le S-morphisme canonique $X_{1}^{\prime} \frac{\|}{Y^{\prime}} X_{2}^{\prime} \rightarrow X_{1} \frac{H}{Y} X_{2} \quad(c f .1 .1 .2(b))$ est étale.

Il faut montrer que si $x$ est un point de $X=X_{1} \frac{\|}{Y} X_{2}$ qui est dans $\left(X_{1} \cap X_{2}\right) \simeq Y$ et $s i \quad x^{\prime}$ est un point de $X^{\prime}=X_{1}^{\prime} \frac{11}{Y^{\prime}} X_{2}^{\prime}$ au-dessus de $x$, alors ${ }^{0} X^{\prime}, X^{\prime}$ est essentiellement étale sur $0_{X, X}$. Pour ce faire, remarquons d'abord que $\operatorname{Spec} O_{X, X}$ (resp. Spec $O_{X^{\prime}, X^{\prime}}$ ) est le schéma recollé de $\operatorname{spec} O_{X_{1}, X}$ (resp. Spec $O_{X_{1}^{\prime}, x^{\prime}}$ ) et $\operatorname{spec} O_{X_{2}, x}$ (resp. Spec $O_{X_{2}^{\prime}, x^{\prime}}$ ) suivant le fermé commun Spec $O_{Y, X}$ (resp. Spec $\left.O_{Y^{\prime}, x^{\prime}}\right)$. Si l'on note le hensélisé strict d'un anneau local $O$ par $\widetilde{O}$, alors il est clair que $\operatorname{spec} \widetilde{\hat{O}_{X, X}}$ (resp. Spec $\widetilde{O_{X^{\prime}, X^{\prime}}}$ ) est le schéma

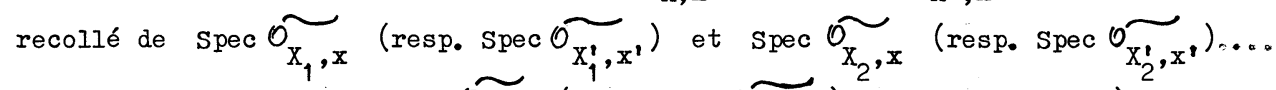
suivant le fermé commun $\operatorname{spec} \widetilde{O_{Y, X}}$ (resp. Spec $\widetilde{O_{Y^{\prime}, X^{\prime}}}$ ). L'assertion iii) est alors immédiate. 
1.2. Application aux questions de descente fpgc sur un schéma de Dedekind

1.2.0. Par définition, un schéma de Dedekind $S$ est un schéma intègre qui est localement le spectre d'un anneau de Dedekind; le schéma $\mathrm{S}$ est donc localement noethérien, intègre et régulier de dimension 1.

Soient $\mathrm{S}$ un schéma de Dedekind, à point générique $\eta$, et $\mathrm{G}:(\mathrm{Sch} / \mathrm{S})^{\circ} \rightarrow$ Ens un foncteur quelconque. On peut définir alors l'adhérence schématique $\bar{G}$ de $G_{\eta}$ dans $G$ comme étant le sous-foncteur $\bar{G}$ de $G$ tel que $T \stackrel{\alpha}{\longrightarrow} G$ est dans $\bar{G}(\mathrm{~T}) \Longleftrightarrow \alpha$ admet une factorisation $\mathrm{T} \stackrel{\alpha}{\longrightarrow} \mathrm{G}$ où $\mathrm{T}^{\prime}$ est un S-schéma plat. $\overbrace{\mathrm{T}^{\prime}}^{\mathrm{T}} \boldsymbol{\alpha}^{\prime}$ où $\mathrm{T}^{\prime}$ est un S-schéma plat.

La motivation de cette définition est assez claire : Pour un schéma quelconque $X$ sur $S$, I'adhérence schématique $\bar{X}$ de $X_{\eta}$ dans $X$ est le plus grand sousschéma de $X$ sans S-torsion, donc est le plus grand sous-schéma plat sur $S$. La définition fonctorielle dit simplement que $\bar{G}$ est "le plus grand sous-foncteur de G plat sur $\mathrm{S} "$.

Il s'ensuit que si G est représentable, les deux définitions coincident. Remarquons enfin que si $\bar{G}$ est représentable, il est nécessairement plat sur $S$.

Pour que le foncteur $G$ soit représentable sur $S$, on a ainsi les conditions nécessaires suivantes :

i) $\vec{G}$, I'adhérence schématique de $G_{\eta}$ dans $G$ est représentable ;

(+) ii) chaque voisinage infinitésimal de chaque fibre de $G$ sur $S$ est représentable ;

iii) G est un S-faisceau fpqc.

On a la réciproque partielle suivante:

1.2.1. Proposition : Soient $s$ un trait $(s, \eta, s), G:(\operatorname{sch} / s)^{\circ} \rightarrow$ Ens un foncteur qui vérifie les conditions (+) ci-dessus. Supposons qu'il existe un trait $S^{\prime}=\left(S^{\prime}, \eta^{\prime}, S^{\prime}\right)$ fidèlement plat sur $S$ tel que $G_{S^{\prime}}$ soit un schéma localement noethérien. Alors $G$ est représentable et localement noethérien. Commençons par quelques remarques. 


\subsubsection{Remarques préliminaires :}

a) Soient $S$ un schéma de Dedekind, $G:(S c h / S)^{\circ} \rightarrow$ Ens un foncteur et $S_{1}$ un schéma de Dedekind plat sur $S$. Si $\bar{G}\left(\operatorname{resp} .\left(\overline{G_{S_{1}}}\right)\right)$ est I'adhérence schématique de $G_{\eta}$ (resp. $\left(G_{S_{1}}\right)$ ) dans $G$ (resp. $G_{S_{1}}$ ) alors on a un isomorphisme canonique $(\bar{G})_{S_{1}} \simeq\left(\overline{G_{S_{1}}}\right)$.

Cela découle formellement des définitions. En d'autres termes, la formation de I'adhérence schématique commute aux changements de base plats.

b) Soient $X$ un schéma sur le trait $S=(S, \eta, s)$ et $\bar{X}$ l'adhérence schématique de $x_{\eta}$ dans $X$. Alors pour chaque $n \geqslant 0, \bar{x} \times{ }_{X^{X}} x_{s}^{(n)}$ est un sous-schéma fermé à la fois de $\bar{X}$ et de $x_{s}^{(n)}$. D'après 1.1 .1 , on peut donc recoller $\bar{X}$ et $x_{s}^{(n)}$ suivant $\bar{x} \times x_{x}^{(n)}$; soit $\bar{x} \Perp x_{s}^{(n)}$ le schéma ainsi obtenu. D'après 1.1.2(b), on a un morphisme canonique $\bar{x} \Perp x_{s}^{(n)} \stackrel{\varphi(n)}{\longrightarrow} X$.

c) On a vu (1.0) que si $X$ est un schéma affine noethérien, c'est un isomorphisme pour $n$ assez grand. On a vu aussi que le recollement suivant un fermé "se fait localement" en se ramenant au cas affine (cf. 1.1.4). Il en résulte que si $X$ est noethérien, $\varphi^{(n)}$ est un isomorphisme pour $n$ assez grand; et par suite, si $X$ est localement noethérien, alors localement sur $X, \varphi^{(n)}$ est un isomor: phisme pour n assez grand .

d) Plaçons-nous dans le contexte de 1.2.1 et supposons (grâce à b) que $S=(s, \eta, s), S^{\prime}=\left(S^{\prime}, \eta^{\prime}, s^{\prime}\right)$ sont des traits. Alors, pour chaque $n \geqslant 0$, le sousfoncteur $\bar{G} \times{ }_{G} G_{S}^{(n)}$ est représentable et est un sous-schéma fermé de $G_{S}^{(n)}$. En effet, si l'indice de ramification du trait $S^{\prime}$ sur $s$ est $r \geqslant 1$, il est clair (compte tenu de a)) que $\left(\bar{G} \times{ }_{G^{G}}{ }^{(n)}\right)_{S^{\prime}} \simeq \bar{G}_{S^{\prime}}, \times_{G_{S^{\prime}}} G_{S^{\prime}}(n r)$. Mais puisque $G_{S^{\prime}}$ est un schéma, le membre à droite est un sous-schéma fermé de $G_{S^{\prime}}^{(n r)}=\left(G_{S}^{(n)}\right)_{S^{\prime}}$; d'où la conclusion par descente fpqc.

e) Ce fait nous permet, sous les hypothèses de 1.2 .1$. , de recoller les schémas $\bar{G}, G \underset{S}{(n)}$, suivant le fermé $\bar{G} \times{ }_{G} G_{S}^{(n)}$, pour chaque $n \geqslant 0$. Notons 
toujours $\bar{G} \Perp G_{\mathrm{S}}^{(n)}$ le schéma ainsi obtenu. D'après 1.1 .5 i) (le recollement suivant un fermé commute aux changements de base plats) il s'ensuit que

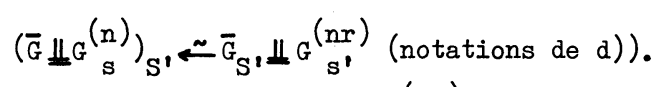

or, on a le morphisme canonique $\bar{G}_{S^{\prime}} \mathbb{\|}_{G^{\prime}}^{(n r)} \stackrel{\varphi^{\prime}(n r)}{\longrightarrow} G_{S^{\prime}}$ et il est formel de voir que les changements de base $S^{\prime \prime}=S^{\prime} \times S^{\prime} \underset{\mathrm{pr}_{2}}{\stackrel{\mathrm{pr}_{1}}{\longrightarrow}} S^{\prime}$ donnent le même morphisme canonique $\quad\left(\bar{G} \Perp_{G}^{(n)}\right)_{S^{\prime \prime}} \rightarrow G_{S^{\prime \prime}} \cdot{ }^{2}$ Comme, par hypothèse, G est un S-faisceau fpqc, on en déduit un morphisme canonique $\bar{G} \Perp_{G} G_{S}^{(n)} \rightarrow G$, pour tout $n \geqslant 0$, que nous noterons $\varphi^{(n)}$; c'est un morphisme relativement représentable par des immersions fermées ; et l'on a des immersions fermées $\bar{G} \underline{\underline{n}} G \underset{\mathrm{S}}{(n)} \rightarrow \bar{G} \mathbb{\Perp} G \underset{\mathrm{S}}{(n+1)}$, compatibles avec les $\varphi^{(n)}, n \geqslant 0$.

1.2.3. Démonstration de 1.2.1:

Remarquons d'abord que les schémas $\bar{G} \mathbb{H}_{\mathrm{G}}^{(\mathrm{n})}, \mathrm{n} \geqslant 0$, ont le même espace sousjacent. Soit $\left\{\sigma_{\alpha}^{(0)}\right\}_{\alpha}$ un recouvrement ouvert affine de $\bar{G} G_{s}^{(0)} ;$ soit, pour chaque $n \geqslant 0$ et chaque $\alpha, U_{\alpha}^{(n)}$ l'ouvert de $\bar{G} \Perp_{G}^{(n)}$ qui a le même espace sousjacent que $U_{\alpha}^{(0)}$; alors $U_{\alpha}^{(n)}$ est affine (EGA, I, 5.1.9), de sorte que $\left\{U_{\alpha}^{(n)}\right\}_{\alpha}$ est un recouvrement ouvert affine de $\bar{G} \Perp_{G}(n)$.

or, considérons, pour a fixe, la famille $\left(\left(U_{\alpha}^{(n)}\right)_{S}\right)_{n \geqslant 0}$. Puisque $G_{S^{\prime}}$ est un schéma localement noethérien, $(1 \cdot 2.2 \mathrm{c})$ ) montre que cette famille est stationnaire et que sa valeur stationnaire est un ouvert du schéma $G_{S^{\prime}}$ - Par descente fidèlement

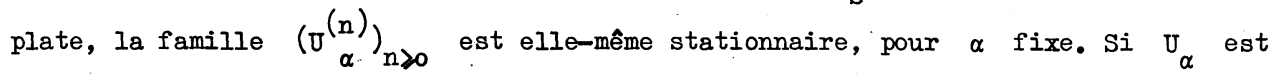
sa valeur stationnaire il est clair que $U_{\alpha}$ représente un ouvert de $G$. Enfin les $\left\{U_{\alpha}\right\}_{\alpha}$ recouvrent G.

1.2.4. Corollaire : Soient $\mathrm{S}$ un schéma de Dedekind et $\mathrm{G}:(\mathrm{Sch} / \mathrm{s})^{\circ} \rightarrow$ Ens un foncteur qui vérifie les conditions $(+)$ de 1.2 .0 et qui est localement de présentar tion finie sur $S$ (cf. EGA, IV, 8.14). Supposons qu'il existe un schéma $S^{\prime}$ fpqc sur $S$ tel que $G_{S^{\prime}}$, soit représentable (et localement de présentation finie) sur $S^{\prime}$. Alors $G$ est représentable. 
Démonstration : Comme plus haut, on peut supposer que $S$ est un trait (Spec $\Lambda, \eta, S)$ et par suite que $S^{\prime}$ est affine, soit $S^{\prime}=$ Spec $A^{\prime}$. On peut évidemment supposer que $A^{\prime}$ est un anneau de valuation (non nécessairement discrète) dominant $\Lambda$. Dans ce cas, l'adhérence schématique (ordinaire) $\overline{G^{\prime}}$ de la fibre générique de $G^{\prime}=G_{S^{\prime}}$ sur $S^{\prime}$, dans $G^{\prime}$, est plate sur $S^{\prime}$, donc on a l'égalité $\overline{G^{\top}} \cong \bar{G} \times{ }_{S^{\prime}} S^{\prime}$. Par descente fpqc on conclut que, pour chaque $n \geqslant 0, \bar{G} \times{ }_{G} G_{S}^{(n)}$ est un sous-schéma fermé de $G_{\mathrm{s}}^{(\mathrm{n})}$.

Ce fait nous permet de construire, suivant le procédé de 1.1 .4$. , des ouverts $\left\{\mathrm{U}_{\alpha}\right\}_{\alpha} \mathrm{du}$ foncteur G tels que :

a) pour tout $\alpha$ et tout $n \geqslant 0, U_{\alpha} n \bar{G}$ (resp. $U_{\alpha} n G{ }_{s}^{(n)}$ ) soit un ouvert affine de $\bar{G} \quad\left(\right.$ resp. $G_{s}^{(n)}$ )

b) $\bigcup_{\alpha} U_{\alpha}=G$.

Puisqu'il suffit de montrer que chacun des ouverts $\left\{U_{\alpha}\right\}$ est un schéma, on peut supposer que $G_{S^{\prime}}$ est de présentation finie sur $S^{\prime}$, dans $I^{\prime e ́ n o n c e ́ ~ 1.2 .4 ~(e n ~ e f f e t ~}$ chaque $\left(U_{\alpha}\right)_{S}$, est un ouvert quasi-compact et quasi-séparé du schéma $\left.G_{S},\right)$. Or, I'anneau $\mathbf{A}^{\prime}$ est limite inductive filtrante de ses sous- $\Lambda$-algèbres $\left\{\mathbf{A}_{i}\right\}_{i \in I}$ de type fini. Puisque $G_{S^{\prime}}$ est de présentation finie sur $S^{\prime}$, il s'ensuit que $G \otimes A_{i}$ est représentable et de type fini sur $\operatorname{spec} A_{i_{0}}$ pour $i_{0} \in I$ assez grand (cf. $[8])$.

Comme $\mathbf{A}^{\prime}$ est sans $\Lambda$-torsion, il en est de même de $\mathbf{A}_{i_{0}}$, donc $\operatorname{Spec} \mathbf{A}_{i_{0}}$ est S-plat. Quitte à prendre une S-quasi-section plate de $\operatorname{spec} A_{i_{0}}$ (cf. EGA, IV, 17.16.1) et par la suite normaliser et localiser, on se ramène à supposer que $S^{\prime}$ est un trait fidèlement plat sur $\mathrm{S}$. On est donc dans un cas particulier de 1.2.1.

C.Q.F.D.

\subsubsection{Remarques: :}

a) Gardons les notations et les hypothèses générales de 1.2.0. Nous dirons que $G$ est plat sur $S$ si $G=\bar{G}$.

b) Il est formel de voir que si G est un foncteur en groupes sur S , alors $\bar{G}$ l'est aussi. 
c) Supposons que la fibre générique $G_{\eta}$ de $G$ sur $S$ est représentable. Si $H_{\eta} \hookrightarrow G_{\eta}$ est un sous-schéma fermé de $G_{\eta}$, alors on a une définition évidente de l'adhérence schématique $\overline{\mathrm{H}}$ de $\mathrm{H}_{\eta}$ dans $G$, qui coincide avec la définition habituelle si $G$ est un schéma.

d) Si, dans c), $G$ est un foncteur en groupes et $\mathrm{H}_{\eta}$ est un sous-groupe invariant fermé de $G_{\eta}$, il est clair que $\overline{\mathrm{H}}$ est un sous-foncteur en groupes invariant de $\bar{G}$.

\subsection{Application aux espaces algébriques}

1.3.0. Définition : Soit $S$ un schéma. On appelle s-espace algébrique un S-faisceau fppf $X$ qui s'insère dans une suite exacte de S-faisceaux $\mathrm{Z} \underset{\mathrm{p}_{2}}{\stackrel{\mathrm{p}_{1}}{\longrightarrow}} \mathrm{Y} \stackrel{\mathrm{p}}{\longrightarrow} \mathrm{X}$, où

i) $Y, Z$ sont des S-schémas,

ii) $\mathrm{Z} \underset{\mathrm{p}_{2}}{\stackrel{\mathrm{p}_{1}}{\longrightarrow}} \mathrm{Y}$ est une S-relation d'équivalence étale et de présentation

finie,

$$
\text { iii) le monomorphisme canonique } \mathrm{Z} \stackrel{\left(\mathrm{p}_{1}, \mathrm{p}_{2}\right)}{\longrightarrow} \mathrm{Y} \times{ }_{\mathrm{S}} \mathrm{Y} \text { est une immersion. }
$$

Nos espaces algébriques sont donc les espaces algébriques localement séparés au sens de $[1]$. $\left(*_{1}\right)$ L'espace algébrique $X$ ci-dessus est dit séparé si le morphisme d : $\mathrm{Z} \stackrel{\left(\mathrm{p}_{1}, \mathrm{p}_{2}\right)}{\longrightarrow} \mathrm{Y} \times{ }_{\mathrm{S}} \mathrm{Y}$ est une immersion fermée. $\left(*_{2}\right)$ Cet espace algébrique $X$ est dit un S-espace algébrique (Ioc. PF) si Y est localement de présentation finie sur $\mathrm{S}$.

\subsubsection{Remarques :}

a) Avec les notations de 1.3.0., soit $\left\{\mathrm{Y}_{\alpha}\right\}_{\alpha}$ un recouvrement ouvert affine de $Y$. Si $Y_{1}$ est le S-schéma $\frac{\|}{\alpha} Y_{\alpha}$ et $Y_{1} \stackrel{\theta}{\rightarrow} Y$ est le morphisme canonique, soit $Z_{1} \rightarrow Y_{1}$ la relation induite sur $Y_{1}$ (cf. SGAD, V, 3). On voit que cette relation est elle aussi étale et de présentation finie (cf.3.1.2.2). Si $X_{1}$ est le S-faisceau fppf quotient de $Z_{1} \rightarrow Y_{1}$, alors il est clair que $X_{1} \cong X$. 
En particulier, on peut supposer dans la définition plus haut que $Y$ est séparé ( $\operatorname{sur} \mathrm{s}$ ).

b) Un S-espace algébrique (loc. PF) X est localement de présentation finie sur $S$ (au sens de EGA, IV, 8.14) (cf. [18], VI, 1.2.1).

c) Supposons maintenant que $\mathrm{S}$ est un schéma de Dedekind. Alors on a la notion d'adhérence schématique $\bar{X}$ de la fibre générique de $X$ dans $X \quad(1.2 .0)$. Si $X$ est un S-espace algébrique, alors $\bar{X}$ est un sous-s-espace algébrique fermé de $X$ : en effet, soit $X$ le conoyau $Z \stackrel{p_{1}}{\overrightarrow{p_{2}}} Y \stackrel{p}{\longrightarrow} X$. Alors la projection canonique $\mathrm{Y} \stackrel{\mathrm{p}}{\rightarrow} \mathrm{X}$ est relativement représentable par des morphismes étales et de présentation finie (cf. 3.1.2.1). On en conclut facilement que $\bar{Y} \cong \bar{X} \times{ }_{X} Y$. D'autre part, considérons la relation d'équivalence induite sur $\bar{Y}$ pour le monomorphisme canonique $\overline{\mathrm{Y}} \rightarrow \mathrm{Y}$. Comme $\mathrm{Z} \cong \mathrm{Y} \times{ }_{\mathrm{X}} \mathrm{Y} \quad(\mathrm{cf} \cdot 3 \cdot 1.2 .1)$ et $\mathrm{p}_{1}, \mathrm{p}_{2}$ sont les projections canoniques $\mathrm{Y} \times{ }_{\mathrm{X}} \mathrm{Y} \rightarrow \mathrm{Y}$, il s'ensuit que cette relation sur $\overline{\mathrm{Y}} \mathrm{n}^{\prime}$ est rien d'autre que $\overline{\mathrm{Z}} \vec{\rightarrow} \overline{\mathrm{Y}}$, interprétés soit comme les projections canoniques $\overline{\mathrm{Z}} \simeq \overline{\mathrm{Y}} \times{ }_{\overline{\mathrm{X}}} \overline{\mathrm{Y}} \rightarrow \overline{\mathrm{Y}}$, soit comme les factorisations des restrictions $p_{i} \mid \bar{Z}$. Il est alors clair que $\bar{Z} \rightarrow \bar{Y}$ est une S-relation d'équivalence étale et de présentation finie. D'autre part, du fait $\bar{Z} \cong \bar{Y} \times{ }_{\bar{X}} \bar{Y}$, on déduit facilement que $\bar{X}$ est le s-faisceau fppf quotient de $\overline{\mathrm{Z}} \rightrightarrows \overline{\mathrm{Y}}$.

Le but de ce numéro est de démontrer la

1.3.2. Proposition : Soient $S$ un schéma de Dedekind et $X$ un S-espace algébrique (loc. PF). Supposons que
i) $\bar{X}$ est représentable (notation de $1.3 .1 \mathrm{c})$ )
et $i i)$ chaque voisinage infinitésimal de chaque fibre spéciale de $X$ sur $S$ est représentable.

Alors $X$ est représentable.

Démonstration : Soit $X$ le conoyau $Z \underset{p_{2}}{\stackrel{p_{1}}{\longrightarrow}} Y \stackrel{p}{\longrightarrow} X$, comme plus haut. Ramenons-nous d'abord au cas $X$ "quasi-compact" ; en effet, si $U$ est un ouvert affine quelconqui de $Y$, son saturé $p_{1} p_{2}^{-1}(U)$ est un ouvert de $Y$ de présentation finie 
sur $S$ et on peut remplacer $Y$ par cet ouvert et $X$ par l'ouvert correspondant. Ramenono-nous par la suite au cas où $S$ est un trait; en effet, puisque $X$ est de présentation finie, il suffit de prouver la représentabilité après localisation en chaque point $s$ de $s$ (cf. [8]). On peut donc supposer que $s=(s, \eta, s)$ est un trait.

Remarquons alors que pour chaque $n \geqslant 0, \bar{X} \times{ }_{X} X_{s}^{(n)}$ est un sous-schéma fermé de $\mathrm{X}_{\mathrm{s}}^{(n)}$, car $\overline{\mathrm{X}} \hookrightarrow \mathrm{X}$ est une immersion fermée (relative). Cela nous permet de construire les schémas $\bar{X} \Perp X_{s}^{(n)}$, pour chaque $n \geqslant 0$ (notations de 1.2.2). On sait (d'après $1.2 .2 \mathrm{c})$ ) qu'il existe $m \geqslant 0$ tel que $\bar{Y} \Perp Y_{\mathrm{S}}^{(\mathrm{m})} \cong \mathrm{Y}$ et $\overline{\mathrm{Z}} \Perp \mathrm{Z}_{\mathrm{s}}^{(\mathrm{m})} \cong \mathrm{Z}$.

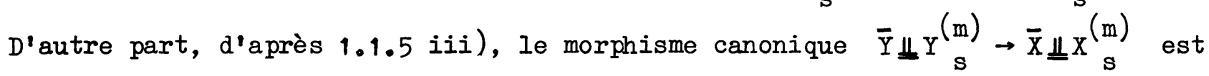

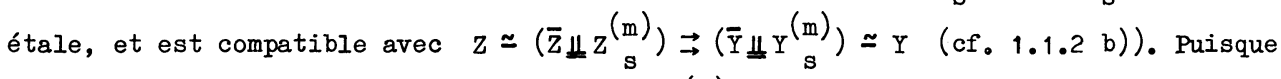
$Z$ a "le bon graphe", on en conclut que $\bar{X} \Perp X_{s}^{(m)}$ s'identifie au faisceau fppf quotient de $\mathrm{Z} \underset{\mathrm{p}_{2}}{\stackrel{\mathrm{p}_{1}}{\longrightarrow}} \mathrm{Y}$. 
Chapitre II

Schémas en groupes sur un schéma de Dedekind

2.0. Nous appellerons semi-trait un schéma de Dedekind semi-local.

La plupart des résultats de ce chapitre concerne les schémas en groupes plats et séparés sur un semi-trait. Certains d'entre eux sont dus à Raynaud.

2.1. La lissification $(N) \quad(N=$ Néron ; cf. $[20])$

2.1.1. Théorème $(N)$ : Soient $S$ un semi-trait à point générique $\eta$ et $G$ un S-schéma en groupes localement de type fini, à fibre générique $G_{\eta}$ lisse. Alors il existe un schéma en groupes $G^{\prime}$ lisse sur $S$ et un S-homomorphisme $u^{\prime}: G^{\prime} \rightarrow G$ avec les propriétés suivantes :

i) $u_{\eta}^{\prime}: G_{\eta}^{\prime} \rightarrow G_{\eta}$ est un isomorphisme

ii) tout $\mathrm{S}$-morphisme $\mathrm{X} \stackrel{\theta}{\longrightarrow} \mathrm{G}$ avec $\mathrm{X}$ S-lisse admet une factorisation unique $\quad x \stackrel{\theta}{\longrightarrow} G$.

De plus, la "formation" du couple ( $\left.G^{\prime}, u^{\prime}\right)$ commute aux changements de base étales sur S .

Nous ne donnerons ici que quelques indications de la démonstration de 2.1.1. 2.1.2. L'idée de la construction du couple $\left(G^{\prime}, u^{\prime}\right)$

On peut supposer que $S$ est un trait ( $\operatorname{Spec} \Lambda, \eta, s)$. L'idée est la suivante : prendre le plus petit sous-schéma en groupes $H$ de $G_{S}$ qui contient les points de $G_{S}$ par lesquels passent une quasi-section étale de $G$ au-dessus de $S$, et faire "grossir" H par certains types "d'éclatements" de G .

Nous commençons donc par spécifier le type d'éclatement que nous allons considérer.

(A) Soit $X$ un schéma localement de type fini sur le trait $S$ et supposons donné un sous-schéma fermé $Y$ de la fibre spéciale $X_{S}$. Soit $E(X, Y)$ le S-schéma 
éclaté de $X$ suivant $Y$. Comme $Y$ est au-dessus de $s$, l'idéal $I$ de $Y$ dans $X$ "contient" $\pi$ (où $\pi$ est une uniformisante de $\Lambda$ ) et on peut considérer l'ouvert $X^{Y}$ de $E(X, Y)$ où $\pi$ engendre l'idéal inversible image inverse de $I$ dans $\mathrm{E}(\mathrm{X}, \mathrm{Y})$.

C'est un schéma $\mathrm{X}^{\mathrm{Y}}$ possédant les propriétés suivantes :

1) $X^{Y}$ est $S-p l a t$, le morphisme canonique $X^{Y} \stackrel{p}{\rightarrow} X$ est affine et $p_{\eta}$ est un isomorphisme.

2) Tout $S$-morphisme $T \stackrel{\alpha}{\longrightarrow} X$ où $T$ est $S-p l a t$ et $\alpha_{S}$ se factorise par $Y$, admet une factorisation unique

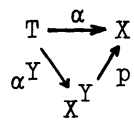

3) Soit $\left(S^{\prime}, \eta^{\prime}, S^{\prime}\right)$ un trait étale sur $S$. Posons $X^{\prime}=X_{S^{\prime}}, Y^{\prime}=Y_{S^{\prime}}$. Alors on a un isomorphisme canonique

$$
\left(\mathrm{X}^{\mathrm{Y}}\right)_{\mathrm{S}^{\prime}} \cong \mathrm{X}^{, \mathrm{Y}^{\prime}}
$$

(B) Revenons à la situation décrite dans 2.1.1. Soient $\pi$ une uniformisante de $\Lambda$ et (Spec $\tilde{\Lambda}=\tilde{S}, \tilde{s}, \tilde{\eta})$ le trait hensélisé strict de $S$ (EGA, IV, 18); posons $\tilde{G}=G_{\tilde{S}}$.

Considérons l'ensemble des points $\left\{g \in \tilde{G}_{\tilde{S}} \mid\right.$ tels qu'il existe une section $\tilde{\mathrm{S}} \rightarrow \tilde{G}$ passant par $g\}$ dans le groupe $\tilde{G}_{\tilde{S}}$. Alors on montre que le plus petit sousschéma fermé $\tilde{H}_{0}$ de $\tilde{G}_{\tilde{s}}$ qui majore cet ensemble est un sous-groupe lisse $\operatorname{sur} k(\tilde{s})$ (SGAD, $\mathrm{VI}_{\mathrm{B}}, 7$ ). Par descente galoisienne on obtient un sous-groupe $\mathrm{H}_{\mathrm{O}} \mathrm{de}_{\mathrm{S}}$, lisse $\operatorname{sur} \mathrm{k}(\mathrm{s})$.

Avec les notations de $(A)$, posons $G_{1}=G^{H^{0}}$; c'es't un schéma qui possède les propriétés correspondantes à 1), 2) et 3) plus haut. D'autre part, en utilisant le fait que $G$ (resp. $\mathrm{H}_{0}$ ) est un groupe, on voit formellement (moyennant 2)) que $G_{1}=G^{H_{0}}$ est aussi un $S$-schéma en groupes et que le morphisme canonique $u_{1}: G_{1} \rightarrow G$ est un $S$-homomorphisme. 
(C) Reprenons le cas général étudié dans $(A)$. Si $x$ est un point de $x(\tilde{S})$ (où $\tilde{S}$ est comme dans $(B))$, alors $\Gamma\left(\tilde{S}, x^{*}\left(\Omega_{X / S}\right)\right)$ est un $\tilde{\Lambda}$-module et on note $m(x, X)$ la longueur de la partie de $\tilde{\Lambda}$-torsion de ce module. On voit alors (cf. [3]) que si X est supposé $S$-1isse au point $x(\tilde{\eta}), x$ est $S$ - lisse au point $x(\tilde{s})$ si et seulement $\underline{\text { si }} \mathrm{m}(\mathrm{x}, \mathrm{X})=0$.

(D) Revenant au cas de (B), par translation, on prouve que le nombre $m(x, G)$ est indépendant $d u$ choix du point $x$ dans $G(\tilde{S})$ (cf. [3]) et nous le noterons $m(G)$. (E) Si $G_{1} \stackrel{u_{1}}{\longrightarrow} G$ est comme dans $(B)$, on a l'inégalité $m\left(G_{1}\right) \leqslant \operatorname{Sup}\{0, m(G)-1\}$ (cf. [3]).

(F) On construit un S-schéma en groupes $G_{2}$ à partir de $G_{1}$ et du sous-groupe fermé lisse $H_{1}$ de $\left(G_{1}\right)_{s}$ obtenu de la même façon que $H_{0}$ dans $G_{s}$ :

$G_{2}=G_{1}{ }_{1}$. Le morphisme canonique $G_{2} \stackrel{u_{2}}{\longrightarrow} G_{1}$ possède pour sa part les propriétés correspondantes à 1), 2), 3) plus haut et est un S-homomorphisme.

En réitérant le processus on finit par trouver un S-groupe $G^{\prime}$ et un $S$-homomorphisme $u^{\prime}: G^{\prime} \rightarrow G$ tels que $m\left(G^{\prime}\right)$ soit zéro (grâce à $(E)$ ), et tel que $u_{\eta}^{\prime}$ soit un isomorphisme (en particulier, $G_{\eta}^{\prime}$ est lisse). Il résulte de (c) alors que G' est S-lisse. Pour "prouver" le théorème (N) il ne reste plus qu'à vérifier la propriété ii) de l'énoncé.

Mais si $X$ est $S$-lisse et $X \stackrel{\theta}{\longrightarrow} G$ est un S-morphisme, alors $\theta_{\mathbf{s}}$ se factorise forcément par $H_{0}$ : en effet, $X_{S}$ est réduit et les points de $X_{s}$ par lesquels passent $S$-morphismes $\tilde{S} \rightarrow X$ sont denses dans $X_{S}$ (EGA, IV,17). D'après $(A)$, 2), donc $\theta$ admet une factorisation unique $\quad x \stackrel{\theta}{\longrightarrow} G$. Le même raisonnement relève $\theta_{1}$ à $G_{2}$ et par itération on prouve ii).

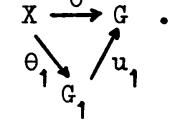

\subsubsection{Remarques :}

(a) Gardons les hypothèses générales de 2.1.1 et supposons que $H_{0}^{\prime}$ est un sous-groupe de $G_{s}$ lisse sur $k(s)$ et tel que l'ensemble des points de $G_{s}$ par lesquels passent des s-morphismes $\tilde{S} \rightarrow G$ soit dense dans $H_{0}^{\prime}$ (i.e., $H_{0}^{\prime}$ est un 
sous-groupe lisse du groupe $\mathrm{H}_{\mathrm{o}}$ qui intervient dans la "démonstration"). Alors le procédé ci-dessus reste entièrement valable et nous fournit une lissification, universelle pour les $S$-morphismes $X \stackrel{\theta}{\longrightarrow} G$, où $X$ est $S$-lisse et $\theta_{S}$ se factorise par $\mathrm{H}_{0}^{\prime}$.

(b) Remarquons enfin que le procédé ainsi que la conclusion du théorème restent valables pour un S-espace homogène $X$ sous $G$ (en effet le point (D) de 2.1.2 n'utilise que I'homogénéité de G); mais ce n'est intéressant que si $X(\tilde{S}) \neq \varnothing$.

\subsection{Une application aux Groupes commutatifs}

Rappelons d'abord que tout schéma en groupes lisse, séparé et de type fini sur un semi-trait est quasi-projectif (cf. [18], VIII.2). L'application que nous avons en vue est la suivante.

2.2.1. Proposition : Soient $\mathrm{S}$ un semi-trait (à point générique $\eta$ ), $\mathrm{G}$ un S-schéma en groupes commutatifs, plat, séparé et de type fini. Alors G est quasiprojectif.

Remarquons tout de suite que l'on peut supposer que $G_{\eta}$ est lisse : en effet, soit $R_{\eta}$ un sous-groupe radiciel de $G_{\eta}$ tel que $G_{\eta} / R_{\eta}$ soit un groupe lisse (SGAD, XVII, Append.II.3.1). Si $R$ est l'adhérence schématique de $R_{\eta}$ dans $G$, alors il est clair que $R$ est fini radiciel et plat sur $S$; donc le faisceau fppf quotient $G_{1}=G / R$ est un schéma (SGAD, $\left.V\right)$ en groupes ( $R$ invariant dans $\left.G\right)$, plat, séparé et de type fini sur $S$; et le morphisme canonique $G \rightarrow G$ est fini (loc. Cit.). Il suffit donc de prouver 2.2.1 pour $G_{1}$; de sorte que l'on peut supposer $G_{\eta}$ lisse.

Dans ce cas la proposition 2.2.1 sera démontrée de la façon suivante : on montrera qu'il existe un entier $n>0$ tel que l'homomorphisme $n_{G}: G \rightarrow G$ (d'élévation à la puissance $\mathrm{n}^{\text {ème }}$ dans G) soit quasi-affine et admette une factorisation<smiles></smiles> 
où $\left(G^{\prime}, u^{\prime}\right)$ est la lissification $(N)$ de $G$ sur $S$; alors $\omega$ est quasi-affine et la proposition résulte du fait rappelé plus haut.

Nous commençons donc par une étude des morphismes $\mathrm{n}_{G}$. Rappelons d'abord deux résultats classiques.

2.2.2. Proposition : Soient $G$ une variété abélienne sur un corps $k, p$ la caractéristique de $k$ et $n$ un entier $>0$. Alors $I^{\prime}$ homomorphisme $n_{G}: G \rightarrow G$

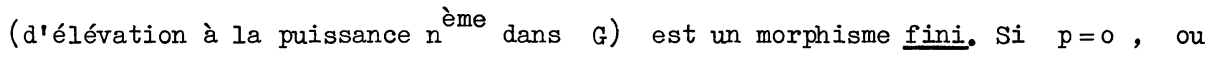
$(n, p)=1$, alors c'est même un morphisme étale.

Pour une démonstration, voir Lang [14], IV, §3, théorème 6, ou Mumford [15] page 62.

2.2.3. Proposition : Soient G un schéma en groupes commutatifs, localement de type fini, sur un corps k, p la caractéristique de $k$ et $n$ en entier $>0$. Si $p=0$, ou $(n, p)=1$, alors $l^{\prime h o m o m o r p h i s m e ~} n_{G}: G \rightarrow G$ est un morphisme étale.

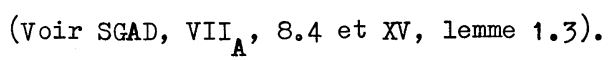

2.2.4. Corollaire de 2.2.2 : Soient G un schéma en groupes commutatifs, de type fini, sur un corps $k$ et $n$ un entier $>0$. Alors l'homomorphisme $n_{G}$ est un morphisme affine.

Démonstration : On peut supposer $k$ algébriquement clos. D'autre part si ${ }_{n}{ }^{G}$ est le noyau de $n_{G}$, on a une factorisation canonique $G \rightarrow G /{ }_{n} G \hookrightarrow G$ de $n_{G}$, où la deuxième flèche est un k-monomorphisme de k-groupes de type fini, donc est une immersion fermée (SGAD, VI ${ }_{B}, 1.4 .2$ ). Il suffit de montrer donc que $G \rightarrow G /{ }_{n} G$ est un morphisme affine, ou ce qui revient au même, que $n^{G}$ est un k-groupe affine.

Or, si $G_{0}$ est la composante neutre de $G, G / G_{0}$ est un k-groupe fini; il suffit donc de prouver que ${ }_{n} G_{0}$ est affine et on peut ainsi supposer $G=G_{0}$. Puisque $k$ est parfait, $G_{\text {red }}$ est un sous-groupe de $G$ et $G / G_{\text {red }}$ est radiciel; un raisonnement analogue nous permet alors de supposer $G=G_{\text {red }}$ •

Mais alors il existe un sous-groupe linéaire $L$ de $G$ tel que $A=G / L$ soit une variété abélienne ([23], théorème 16). Puisque $n^{A}$ est fini (2.2.2) et que ${ }_{n}{ }^{L}$ est linéaire, le corollaire est prouvé. 


\subsubsection{Remarques :}

(a) Soient $S$ un schéma, $G$ un S-schéma en groupes commutatifs, plat et de présentation finie sur $\mathrm{S}$ et $\mathrm{n}$ un entier $>0$ inversible sur $\mathrm{S}$. Alors il résulte de 2.2.3 que $n_{G}$ est un morphisme étale et de présentation finie (EGA, IV, 11.3.10); en particulier, ${ }_{n}^{G}=\operatorname{ker}\left(n_{G}\right)$ est un S-groupe étale et de présentation finie.

(b) Si, de plus, les fibres de G sur $\mathrm{S}$ sont supposées connexes (donc géométriquement irréductibles), il s'ensuit que $\mathrm{n}_{\mathrm{G}}$ est un morphisme fidèlement plat. 2.2.6. Soit $k$ un corps de caractéristique $p \geqslant 0$. (Pour un entier $n>0$ nous adoptons la convention que $(n, p)=1$ est une condition vide si $p=0)$. On vient de voir que si $n$ est un entier $>0$ tel que $(n, p)=1$, alors $n^{G}$ est un k-groupe étale. Il en résulte que le plus petit sous-schéma fermé $\mathrm{H}$ de $\mathrm{G}$ qui ma-

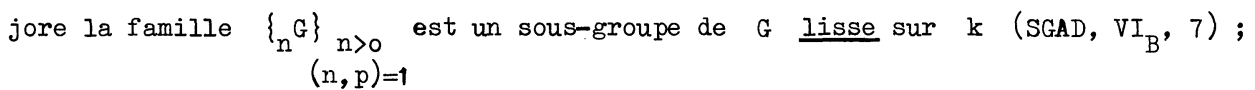
il est facile de voir que la formation de $\mathrm{H}$ commute aux extensions du corps de base (Ioc. cit.).

Soit $\overline{\mathrm{k}}$ une clôture algébrique de $\mathrm{k}$. Pour le reste de ce numéro, nous nous bornons aux points géométriques de G,H... à valeurs dans $\overline{\mathrm{k}}$.

i) Soit $x \in G(\bar{k})$. Si, pour un $n>0,(n, p)=1$, on a $x^{n} \in H(\bar{k})$ alors $\mathrm{x} \in \mathrm{H}(\overline{\mathrm{k}})$.

Cas a). H connexe. Il résulte de 2.2.5 (b) que $n_{H}$ est surjectif. On a donc $x^{n}=y^{n}, y \in H, \quad d^{\prime}$ où $x \in H(\bar{k})$.

Cas b). H non connexe. Le même raisonnement montre que l'ordre du groupe fini $\mathrm{H} / \mathrm{H}_{\mathrm{O}}$ $\left(H_{0}=\right.$ composante neutre de $\left.H\right)$ est un entier $m$ tel que $(m, p)=1$. Donc si $x^{n} \in H(\bar{k}),\left(x^{n}\right)^{m} \in H_{0}(\bar{k})$ d'où, comme avant, $x \in H(\bar{k})$.

ii) Il résulte de i) que ${ }_{n}(G / H)=(0)$, pour tout $n>0,(n, p)=1$. iii) Il résulte de $i i)$ que si $G$ est une variété abélienne, alors $G=H$ : en effet, G/H est une variété abélienne et l'assertion résulte donc de 2.2.2 . 
iv) Soient $\mathrm{L}$ un $\mathrm{k}$-sous-groupe (invariant) connexe de $\mathrm{G}$ et $\mathbf{A}=\mathrm{G} / \mathrm{L}$. Alors pour tout $n>0,(n, p)=1$, le morphisme canonique ${ }_{n} G \rightarrow n^{A}$ est surjectif. Cela résulte du fait que $n_{I}: I \rightarrow I$ est surjectif. Nous sommes maintenant en mesure de prouver la

2.2.7. Proposition : Soient $k$ un corps de caractéristique $p>0$, $\mathrm{g}$ un, k-schéma en groupes commutatifs de type fini et $\mathrm{H}$ le sous-groupe (fermé) de G défini comme dans 2.2.6. Alors il existe un entier $n>0$ tel que $G / H$ soit annulé par $p^{n}$ (i.e., le morphisme $p_{G}^{n}$ se factorise par $H$ ).

Démonstration : En remplaçant $\mathrm{G}$ par G/H, on est ramené, grâce à 2.2 .6 ii), à montrer que si ${ }_{n} G=(0)$ pour tout $n>0,(n, p)=1$, alors $G$ est annulé par une puissance de $\mathrm{p}$. On peut supposer $\mathbf{k}$ algébriquement clos.

Si $G_{0}$ est la composante neutre de $G$, alors (du fait que $n_{G_{0}}$ est surjectif pour $n>0,(n, p)=1$ et $n^{G}=(0)$ pour tout $\left.n>0,(n, p)=1\right)$ on voit que $G / G_{0}$ est un p-groupe, donc annulé par une puissance de $p$. Puisqu'il en est ainsi aussi du k-groupe radiciel $G_{0} /\left(G_{0}\right)_{\text {red }}$, on s'est ramené à supposer $G=G_{0}=\left(G_{0}\right)_{\text {red }}$.

Mais alors il existe un sous-groupe linéaire et connexe $I$ de $G$ tel que $A=G / L$ soit une variété abélienne (cf. [23], théorème 16). Il résulte donc de 2.2.6 iii) et iv) que $\mathrm{G}=\mathrm{I}$, i.e., $\mathrm{G}$ est linéaire et commutatif. La proposition résulte alors du théorème de structure de tels groupes (cf. [24], exposé 12). C.Q.F.D. 2.2.8. Corollaire : Soient $(S, \eta, s)$ un trait, $G$ un $S$-schéma en groupes commutatifs plat et de type fini, à fibre générique lisse, et $H_{0}$ le sous-groupe (fermé) de $G_{s}$ défini comme dans 2.1 .2 (i.e., l'adhérence schématique des images, dans $G_{s}$, des quasi-sections étales de G). SSi $p>0$ est la caractéristique de $k(s)$, alors il existe $n>0$ tel que $G_{s} / H_{0}$ soit annulé par $p^{n}$.

Il suffit de prouver que $H_{0}$ est plus grand que le sous-groupe $H$ de $G_{s}$ défini cọme dans 2.2.6 (grâce à 2.2.7). Pour ce faire, il suffit de remarquer que si $(n, p)=1$, alors $n$ est inversible sur $s$, donc $n^{G}$ est un $s$-groupe étale 
(cf. 2.2.5 (a)).

2.2.9. Corollaire : Avec les hypothèses de 2.2.8., il existe un entier $n>0$ tel que $p_{G}^{n}$ admette une factorisation (unique)

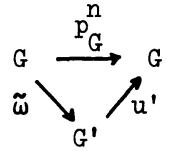

où $\left(G^{\prime}, u^{\prime}\right)$ est la lissification $(N)$ de $G$ sur $S$ et $\tilde{\omega}$ est un S-homomorphisme. Cela résulte de 2.2 .8 et de la propriété universelle de $\left(G^{\prime}, u^{\prime}\right)$ (voir la construction de 2.1.2).

2.2.10. Corollaire : Soient $\mathrm{S}$ un semi-trait, $\mathrm{G}$ un S-schéma en groupes commutatifs, plat et de type fini, à fibre générique lisse et $\left(G^{\prime}, u^{\prime}\right)$ sa lissification (N) sur S. Alors il existe un S-endomorphisme $\varphi$ de G qui admet une factorisation (unique)

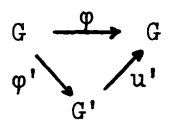

où $\varphi^{\prime}$ est un homomorphisme avec $\operatorname{ker}\left(\varphi_{\eta}^{\prime}\right)$ affine ( $\eta$ étant le point générique de S).

En effet, si $p_{1}, \ldots, p_{r}$ sont les caractéristiques résiduelles de $S$, différentes de zéro, alors on voit qu'il existe $n>0$ tel que $\left(p_{1} \ldots p_{r}\right)_{G}^{n}$ satisfasse à la première assertion. La deuxième assertion résulte alors de 2.2.4.

Remarquons maintenant que pour prouver 2.2.1 (N.B. on s'est ramené au cas $G_{\eta}$ lisse) il ne reste plus qu'à montrer, dans 2.2.10., que $\varphi^{\prime}$ est un morphisme quasiaffine, $\underbrace{\text { lorsque }}_{G}$ est séparé. Ce sera fait dans la section suivante ; en fait nous montrerons que c'est un morphisme affine (cf. 2.3.2). 


\subsection{Schémas en groupes et morphismes affines}

Les deux résultats principaux que nous avons en vue sont les suivants.

2.3.1. Proposition : Soient $S$ un schéma de Dédekind, G un S-schéma en groupes plat, séparé et de type fini sur $S$. Si la fibre générique $G_{\eta}$ de $G$ sur $S$ est affine, alors $G$ est lui-même affine sur $S$.

2.3.2. Proposition : Soient $S$ un schéma de Dédekind, $G, G_{0}$ deux S-schémas en groupes de type fini et $u: G \rightarrow G_{0}$ un S-homomorphisme. On suppose que :
i) u est séparé
ii) G est S-plat

iii) $\operatorname{ker}\left(u_{\eta}\right)$ est affine ( $\eta$ étant le point générique de $s$ ).

Alors $u$ est un morphisme affine.

2.3.3. Démonstration de 2.3.1 : on peut supposer que $s$ est un trait $(s, \eta, s)$. Soit $X$ un S-schéma quasi-compact, quasi-séparé et plat ; alors l'algèbre $T(X)$ est sans torsion sur $S$, donc Spec $T(X)$ est un schéma $S$-plat. On a un morphisme canonique $\mathrm{X} \stackrel{\text { can. }}{\longrightarrow} \mathrm{Spec} \Gamma(\mathrm{X})$ sur $\mathrm{S}$. Alors

2.3.3.1. Lemme : Avec les notations précédentes, le morphisme $\mathrm{X} \stackrel{\text { can. }}{\longrightarrow} \operatorname{Spec} \Gamma(x)$ est schématiquement dominant (EGA, IV, 11) sur chacune des S-fibres.

Au-dessus de $\eta$ c'est clair; et au-dessus de $s$, il s'agit de prouver que, pour tout $f \in \Gamma(x)$, le morphisme canonique $\frac{\Gamma(x)_{f}}{\pi \Gamma(X)_{f}} \rightarrow \Gamma\left(\left(x_{f}\right)_{s}, O_{\left(x_{f}\right)_{s}}\right)$, (où $\pi$ est une uniformisante sur $S$ ), est injectif. Or, puisque $X_{f}$ est $S-p l a t$, on a la suite exacte des faisceaux

$$
0 \rightarrow O_{X_{f}} \stackrel{\pi}{\rightarrow} O_{X_{f}} \rightarrow O_{\left(X_{f}\right)_{s}} \rightarrow 0
$$

D'où la suite exacte

$$
0 \rightarrow \Gamma\left(O_{X_{f}}\right) \stackrel{\pi}{\rightarrow} \Gamma\left(O_{X_{f}}\right) \rightarrow \Gamma\left(O_{\left(X_{f}\right)_{s}}\right) \rightarrow \cdots \cdots
$$

ce qui montre que I'application $\frac{\Gamma\left(X_{f}\right)}{\pi \Gamma\left(X_{f}\right)} \hookrightarrow \Gamma\left(\left(x_{f}\right)_{s}\right)$ est injective; pour finir, on note que $\Gamma\left(x_{f}\right)=\Gamma(x)_{f}$. 
(b) Supposons maintenant que $G$ est un schéma en groupes de type fini et plat sur le trait $(s, \eta, s)$. Considérons le $S$-schéma plat $\operatorname{Spec} \Gamma(G)=G_{1}$ et le morphisme'canonique $G \stackrel{\varphi}{\longrightarrow} G_{1}$. Puisque $G$ est un $S$-groupe et que $G$ est plat, on voit formellement, moyennant la formule de künneth, que $G_{1}$ est un S-groupe et que $\varphi$ est un S-homomorphisme. On vient de voir que $\varphi$ est universellement schématiquement dominant par rapport à $S$.

(c) Nous allons montrer que si $G_{\eta}$ est affine, alors ce morphisme $\varphi$ est étale, surjectif, et est un isomorphisme si G est séparé. Pour ce faire nous aurons besoin $d u$

2.3.3.2. Lemme : Soient $\mathrm{k}$ un corps, $\mathrm{G}$ un $\mathrm{k}$-schéma en groupes de type fini, $G^{\prime}$ un k-groupe quelconque limite projective $\underline{d^{\prime} u n}$ système filtrant $\left(G_{i}^{\prime}, \varphi_{i j}^{\prime}\right)_{i, j \in I}$ de $k$-groupes de type fini, et $u: G \rightarrow G^{\prime}$ un $k$-homomorphisme schématiquement dominant. Alors $u$ est fidèlement plat et $G^{\prime}$ est de type fini.

Démonstration : Si $K=\operatorname{ker}(u)$, alors $K$ est un k-groupe de type fini. Si $u_{i}$ est le k-homomorphisme composé $G \stackrel{u}{\longrightarrow} G^{\prime} \longrightarrow G_{i}^{\prime}$, alors $\left\{K_{i}=\operatorname{ker}\left(u_{i}\right)\right\}_{i \in I}$ est un système décroissant de k-sous-groupes de $G$, donc stationnaire. On voit donc que $K=K_{i_{0}}$ pour un $i_{0}$ assez grand. D'où une factorisation canonique de $u$ comme suit :

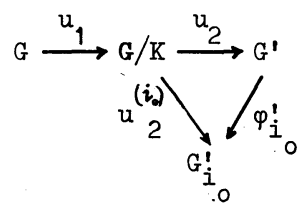

où $u_{1}$ est fidèlement plat, $G / K$ est un k-groupe de type fini et $u_{2}{ }^{\left(i_{0}\right)}$ $u_{1}$ est fidelement plat, $G / x$ est un k-groupe de type fini et $u_{2}, u_{2}{ }^{2}$ sont des k-monomorphismes de groupes. Comme $G_{i}^{\prime}$ est de type fini, $u_{2}$ est une immersion fermée ; puisque $G^{\prime}$ est un k-groupe, G' est séparé (cf. SGAD, VI $^{\text {) }}$ donc $\varphi_{i_{0}}^{\prime}$ est séparé et $i l$ en résulte que $u_{2}$ est une immersion fermée. Mais $u$ est schématiquement dominant, donc $u_{2}$ l'est aussi, d'où le fait que $u_{2}$ est un isomorphisme. 
2.3.3.3. Corollaire : Soient $\mathbf{k}$ un corps, $\mathrm{G}$ un $\mathrm{k}$-groupe de type fini, G un $k$-groupe affine quelconque et $u: G \rightarrow G^{\prime}$ un $k$-homomorphisme schématiquement dominant. Alors $u$ est fidèlement plat et $G^{\prime}$ est de type fini.

En effet un k-groupe affine est limite projective d'un système filtrant de k-groupes affines et de type fini ( $\mathrm{SGAD}, \mathrm{VI}_{B}, 11.5$ ); il suffit alors d'appliquer 2.3 .3 .2

Ceci étant, revenons au contexte de 2.3.1. On a un s-homomorphisme $G \stackrel{\varphi}{\longrightarrow} G_{1}=\operatorname{Spec} \Gamma(G)$, universellement schématiquement dominant (relativement à $S$ ) et par hypothèse $\varphi_{\eta}$ est un isomorphisme. Grâce à 2.3.3.3., on déduit que $\varphi_{\mathbf{s}}$ est fidèlement plat et que $G_{1}$. est de type fini. Puisque $G$ est $S-p l a t$, il s'ensuit que, pour chaque $n \geqslant 0$, le morphisme $\varphi_{s}^{(n)}: G{ }_{s}^{(n)} \rightarrow G_{1}^{(n)}$ est fidèlement plat (EGA, $\left.O_{I I I}, 10.1 .2\right)$ (notations évidentes).

Si $K=\operatorname{ker}(\varphi)$, cela signifie que, pour chaque $n>0, K_{s}^{(n)}$ est $s_{s}^{(n)}-$ plat. Comme $K$ est un S-groupe de type fini, il s'ensuit que $K$ est S-plat (EGA,

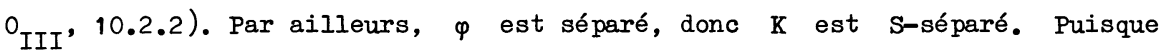
$\mathrm{K}_{\eta}=(0)$, on en conclut que $\mathrm{K}=(0)$. Le morphisme de type fini $\varphi: G \rightarrow G_{1}$ est ainsi un monomorphisme, donc quasi-affine (EGA, IV, 18.12). Mais alors, le morphisme canonique $\varphi$ est une immersion ouverte (EGA, II, 5.1.6). Il en résulte que $\varphi$ est un isomorphisme.

C.Q.F.D.

2.3.3.4. Une digression : Soient $k$ un corps, $G$ un $k$-groupe de type fini. Alors les méthodes employées dans cette section montrent que $G_{1}=\operatorname{Spec} \Gamma(G)$ est un k-groupe, que le morphisme canonique $\varphi: G \rightarrow G_{1}$ est un k-homomorphisme schématiquement dominant, donc fidèlement plat et que $G_{1}$ est de type fini. Il en résulte que $G_{1}$ est un k-groupe linéaire; par suite, c'est le plus grand quotient linéaire de $G$ (mieux : tout $k$-morphisme $G \rightarrow T$ où $T$ est affine se factorise par $\left.G_{1}\right)$. Si $D$ est le sous-groupe $\operatorname{ker}(\varphi)$ de $G$ et $D_{0}$ est la composante neutre de $D, G / D_{0}$ est linéaire, donc $D=D_{0} \cdot$ Enfin si $G$ est connexe, $G / \underline{\operatorname{Cent}}_{k}(G)$ est linéaire $\left([23]\right.$, th.13) donc $D G \underline{\text { Cent }}_{k}(G)$. On retrouve ainsi le 
théorème classique de Rosenlicht ([23], cor.3, th.12).

2.3.4. Démonstration de 2.3.2 : on peut supposer toujours que $s=(s, \eta, s)$ est un trait. Quitte à remplacer $G_{0}$ par l'adhérence schématique de $u_{\eta}\left(G_{\eta}\right)$ on peut supposer que $u_{\eta}$ est fidèlèment plat.

Considérons le sous-groupe fermé $H_{0}=u_{s}\left(G_{S}\right)$ de $G_{0}$ et posons $G_{1}=G_{0}{ }^{H}$ (notations de $2.1 .2(\mathbb{A})$ ). Alors $G_{1}$ est un S-schéma en groupes plat et de type fini sur $S$ et affine sur $G_{0}$. Et $l$ 'homomorphisme $u: G \rightarrow G_{0}$ se relève en un S-homomorphisme $u_{1}: G \rightarrow G_{1}$ de façon unique.

En itérant ce procédé, on trouve un système projectif $\left\{G_{i}\right\}_{i>1}$ de S-schémas en groupes plats et de type fini sur $S$ et affines sur $G_{0}$, et des relèvements compatibles $u_{i}: G \rightarrow G_{i}$ de $u$. Comme les $G_{i}$ sont $G_{0}$-affines, on peut construire le schéma $G_{\infty}=\underset{i}{\lim } G_{i}$; c'est un $S$-schéma en groupes plat sur $S$ et affine sur $G_{0}$; et on a un S-homomorphisme $G \stackrel{u_{\infty}}{\longrightarrow} G_{\infty}$ qui relève les $u_{i}$. (a) Le morphisme $u_{\infty}$ est schématiquement dominant sur chacune des

S- $\underline{\text { fibres. }}$.

Au-dessus de $\eta$ cela résulte du fait que $u_{\eta}$ est fidèlement plat. Au-dessus de $\mathbf{s}$, il s'agit de prouver la chose suivante :

Soient $U_{\infty}$ un ouvert affine de $G_{\infty}$ et $U$ son image réciproque dans $G$. Alors le morphisme canonique composé

$$
\frac{\Gamma\left(U_{\infty}, G_{\infty}\right)}{\pi \Gamma\left(U_{\infty}, G_{\infty}\right)} \rightarrow \frac{\Gamma(U, G)}{\pi \Gamma(U, G)} \hookrightarrow \Gamma\left(U_{s}, G_{s}\right)
$$

est injectif.

En effet, $U_{\infty}$ provient d'un ouvert affine $U_{j}$ de $G_{j}$ pour tout $j$ assez grand; soit $r \in \Gamma\left(U_{\infty}, G_{\infty}\right)$ un élément dont l'image est zéro dans $\Gamma\left(U_{s}, G_{s}\right)$. Par définition, $r$ provient d'un $r_{i} \in \Gamma\left(U_{i}, G_{i}\right)$; il en résulte que $r_{i}$ est dans l'idéal de définition de $H_{i}=u_{i}\left(G_{s}\right)$. Donc l'image $r_{i+1}$ de $r_{i}$ dans $\Gamma\left(U_{i+1}, G_{i+1}\right)$ est dans l'idéal $\pi \Gamma\left(U_{i+1}, G_{i+1}\right)$; d'où l'assertion. 
(b) Il résulte de (a) et de 2.3.3.2 que $\left(u_{\infty}\right)_{s}$ est fidèlement plat et que $\left(G_{\infty}\right)_{s}$ est de type fini. On en conclut, comme dans la démonstration de 2.3.1 que le noyau $K$ de $u_{\infty}$ est S-plat. Par ailleurs $K$ est séparé et de type fini sur $S$. Comme, par hypothèse, $K_{\eta}$ est affine, il résulte de 2.3 .1 que $K$ est affine.

(c) Le S-faisceau quotient $G / K$ est un $S$-schéma.

Cela résulte de [17], p. 294-12.

(d) Si $K_{i}=\operatorname{ker}\left(u_{i}\right)$, on voit que $K=K_{i}$ pour $i$ assez grand. Puisqu'il suffit de montrer que le morphisme induit $G / K \rightarrow G_{\infty}$ est affine, on peut supposer dès abord [quitte à remplacer $G_{0}$ par un $G_{i}$ (pour $i$ assez grand) et $G$ par $G / K]$ que $u$ est un monomorphisme. Il s'ensuit que $u_{\infty}$ est alors quasi-affine; donc, dans la factorisation canonique de $u_{\infty}$

$$
\mathrm{G} \stackrel{\mathrm{v}}{\longrightarrow} \operatorname{Spec}\left(u_{\infty}\right) *\left(\mathcal{O}_{G}\right) \stackrel{\omega}{\longrightarrow} G_{\infty}
$$

$\mathrm{v}$ est une immersion ouverte (EGA, II, 5.1.6).

D'autre part on a vu que $\left(G_{\infty}\right)_{s}$ est de type fini (cf. $\left.(a)\right)$, de sorte que le monomorphisme $\left(u_{\infty}\right)_{s}$ est une immersion ferméé; donc $v_{s}$ est une immersion fermée (tandis que $v_{\eta}$ est un isomorphisme).

Enfin le même raisonnement que dans le lemme 2.3.3.1 montre que $v_{s}$ schématiquement dominant. D'où le fait que $\mathrm{v}$ est un isomorphisme. C.Q.F.D。

2.3.5. Remarque (corollaire) : Soient $S$ ur semi-trait, $\eta$ son point générique, et $G$ un S-schéma en groupes plat, séparé et de type fini, tel que $G_{\eta}$ soit connexe. Alors G est quasi-projectif.

En effet, on se ramène d'abord au cas $G_{\eta}$ lisse comme dans le cas $G$ commutatif. Par suite, il existe un sous-groupe linéaire et invariant $I_{\eta}$ de $G_{\eta}$ tel que $G_{\eta} / L_{\eta}$ soit une variété abélienne. Si $L$ est I'adhérence schématique de $I_{\eta}$ dans $G$, nous montrerons au chapitre IV que $G_{1}=G / L$ est un schéma (en groupes) (cf. Théorème (4.c), chap. IV). Il suffit alors d'appliquer 2.21 et 2.3.2. 
Chapitre III

$\underline{\text { Sur }}$ les faisceaux quotients et les espaces algébriques

\subsection{Un théorème d'Artin}

Soient $S$ un schéma, $Y, Z$ des S-schémas localement de présentation finie et $Z \underset{p_{2}}{\stackrel{p_{1}}{\longrightarrow}} Y$ une s-relation d'équivalence plate et de présentation finie. Notons $\mathrm{X}$ le $\mathrm{S}$-faisceau fppf quotient. Le résultat suivant a été signalé par M. Artin, cf. $[1], 7.1$.

3.1.1. Théorème : Avec les notations précédentes, supposons que $S$ est localement noethérien. Alors $X$ possède une structure de S-espace algébrique (au sens de [1]) localement de présentation finie.

Notre premier objectif dans ce chapitre est de démontrer ce théorème. Grâce au corollaire 3 de l'appendice $I$, on peut supposer que $z \stackrel{p_{1}}{\stackrel{p_{2}}{\longrightarrow}} Y$ est une relation d'équivalence plate, quasi-finie et de présentation finie; et nous $\underline{\text { la }}$ supposerons de $\underline{\text { ce }}$ type dans $\underline{\text { ce }}$ qui suit.

3.1.2. Démonstration de 3.1.1 : Nous aurons besoin de quelques préliminaires.

3.1.2.1. Proposition : Avec les hypothèses et les notations ci-dessus, le carré

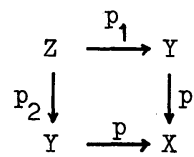

est cartésien ; et la projection canonique $\mathrm{p}: \mathrm{Y} \rightarrow \mathrm{X}$ est relativement représentable par des morphismes quasi-finis et fppf.

Démonstration : La première assertion résulte des généralités faisceautiques. Pour la deuxième, il s'agit de prouver que, si $T \in(\mathrm{Sch} / \mathrm{S})$ et si $\alpha \in X(T)$, alors le S-faisceau $F=T \times{ }_{X} Y$ est représentable, quasi-fini et fppf sur $T$.

L'assertion étant locale sur $T$, on peut supposer $T$ affine. D'autre part, il existe un S-schéma $T^{\prime}$, un $S$-morphisme affine et $f$ ppf $T^{\prime} \stackrel{p^{\prime}}{\longrightarrow} T$, et un diagramme commutatif 


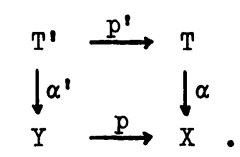

Considérons alors le S-faisceau $\mathrm{F}^{\prime}=\mathrm{F} \times \mathrm{T}_{\mathrm{T}^{\prime}} \mathrm{T}^{\prime} ;$ on a $\mathrm{F}^{\prime}=\mathrm{Y} \times \mathrm{X}^{\mathrm{T}^{\prime}}=\left(\mathrm{Y} \times \mathrm{X}_{\left(\mathrm{Pr}_{2}, \alpha^{\prime}\right)}^{\mathrm{Y})} \times\right.$ $=\mathrm{Z} \times \mathrm{T}^{\prime}$, donc $\mathrm{c}^{\prime}$ est un $\mathrm{S}$-schéma tel que la projection canonique $\mathrm{F}^{\prime} \rightarrow \mathrm{T}^{\prime}$ soit $\left(P_{2}, \alpha^{\prime}\right)$ quasi-finie et fppf ( $\operatorname{car} \mathrm{p}_{2}$ l'est). On est ainsi ramené à "descendre" F' relativement au morphisme fppf $p^{\prime}: T^{\prime} \rightarrow T$.

Soit $U$ un ouvert affine quelconque de $Y$; alors $U \times{ }_{X}^{T}$ est un ouvert de $F=Y \times{ }_{X}^{T}$, donc son image inverse $U \times X_{X^{T^{\prime}}}$ est un ouvert de $F^{\prime}=Y \times X^{T^{\prime}}$ stable pour la donnée de descente canonique sur $F^{\prime}$ (relativement à p'). Par ailleurs, c'est un sous-objet de $U \times{ }_{S} T^{\prime}$, donc il est $S$-séparé. Enfin puisque $p^{\prime}$ est affine et fppf, on peut recouvrir l'ouvert $U \times X_{X^{\prime}}$ de $F^{\prime}$ par des ouverts quasicompacts et stables pour la donnée de descente; ces ouverts seront donc séparés, quasi-finis et quasi-compacts sur T', donc quasi-affines sur T' (EGA, IV, 18). L'effectivité de la donnée de descente sur $F^{\prime}$ est alors assurée (SGA, 1, VIII). C.Q.F.D.

3.1.2.2. Corollaire : Soient $Y^{\prime} \stackrel{u}{\longrightarrow} Y$ un $S-m o r p h i s m e ~ p l a t$ (resp. plat et de présentation finie,...) et $Z^{\prime} \rightarrow Y^{\prime}$ la relation induite sur $Y^{\prime}$ par $Z \Rightarrow Y$ (cf. SGAD, V, 3). Alors c'est une relation d'équivalence plate (resp. plate et de présentation finie,....).

En effet, puisque $\mathrm{Z} \simeq \mathrm{Y} \times{ }_{\mathrm{X}} \mathrm{Y}$, la relation induite sur $Y^{\prime} \mathrm{n}^{\prime}$ est rien d'autre que $Y^{\prime} \times X^{\prime} \stackrel{\mathrm{pr}_{1}}{\stackrel{\mathrm{pr}_{2}}{\longrightarrow}} Y^{\prime}$; d'autre part on a les carrés cartésiens

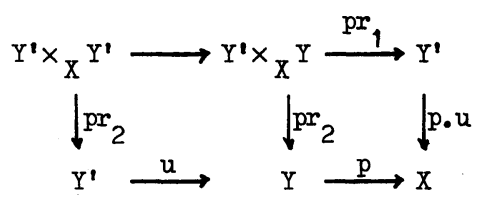

d'où l'assertion. 
3.1.2.3. Corollaire : Soient $\mathrm{T}$ un S-schéma localement de type fini et $\alpha \in X(T)$. Alors l'ensemble $\operatorname{Et}(\alpha)$ des points $t \in T$ où $\alpha$ est (formellement infinitésimalement) étale (SGAD, XI, 1.8) est ouvert dans T. (N.B. L'hypothèse "noethérienne" sur $\mathrm{S}$ est pour la première fois utilisée ici).

En effet, d'après 3.1.2.1., $\mathrm{T} \times{ }_{\mathrm{X}} \mathrm{Y}$ est un schéma fppf sur $\mathrm{T}$ et la projection canonique $\mathrm{T} \times \mathrm{X} \stackrel{\mathrm{pr}_{2}}{\longrightarrow} \mathrm{Y}$ est localement de type fini. Il en résulte que l'ensemble $\boldsymbol{E}_{\mathrm{t}}\left(\mathrm{pr}_{2}\right)$ des points de $\mathrm{T} \times{ }_{\mathrm{X}} \mathrm{Y}$ où $\mathrm{pr}_{2}$ est (formellement infinitésimalement) étale est un ouvert de $\mathrm{T} \times{ }_{\mathrm{X}} \mathrm{Y}$. Il est alors clair que $\mathcal{E}_{t}(\alpha)$ est l'image de $\varepsilon_{t}\left(\mathrm{pr}_{2}\right)$ par le morphisme $f \mathrm{ppf} T \times_{X} \mathrm{Y} \rightarrow \mathrm{T}$, donc est un ouvert de $\mathrm{T}$.

3.1.2.4. Corollaire : On peut généraliser (dans une certaine mesure) 3.1.2.1 comme suit :

Soient $\mathrm{T}_{1}, \mathrm{~T}_{2} \in(\mathrm{Sch} / \mathrm{S})$ et $\alpha_{i} \in \mathrm{X}\left(\mathrm{T}_{i}\right), i=1,2$. Alors $\mathrm{T}_{1} \times \mathrm{X}_{2}$ est un S-schéma.

L'assertion est locale sur $X$ donc on peut supposer $Y$ affine; de même on peut supposer $\mathrm{T}_{1}, \mathrm{~T}_{2}$ affines. On a que $\mathrm{T}_{2} \times_{X} \mathrm{Y}$ est un schéma quasi-affine sur $\mathrm{T}_{2}$; et $\mathrm{Y}$ étant séparé la projection canonique $\mathrm{pr}_{2}: \mathrm{T}_{2} \mathrm{X}_{\mathrm{X}} \mathrm{Y} \rightarrow \mathrm{Y}$ est alors quasiaffine. D'autre part on a un $T_{1}^{\prime} \in(\mathrm{Sch} / \mathrm{S})$, un S-morphisme affine et fppf $\mathrm{T}_{1} \stackrel{\mathrm{p}^{\prime}}{\longrightarrow} \mathrm{T}_{1}$ et un diagramme commutatif

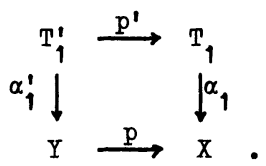

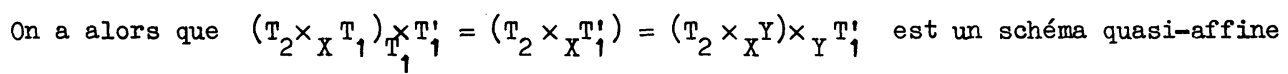
sur $T_{1}^{\prime}$ - D'où la conclusion par descente fpqc.

Après ces préliminaires, revenons aux détails de la démonstration de 3.1 .1 . Soit $y$ un point quelconque de $Y$, fermé dans sa fibre sur $S$. Alors chaque point de son orbite sous $\left(p_{1}, p_{2}\right)$ est un point fermé de la même fibre de $Y$ sur $S$. Comme cette orbite $p_{1} p_{2}^{-1}(y)$ est finie par hypothèse, il existe un ouvert affine $U$ de $Y$ tel que $\left\{p_{1} p_{2}^{-1}(y)\right\} \cap U=\{y\}$. Pour étudier le faisceau $X$ au voisinage de 
l'image de $\mathrm{y}$, on peut remplacer $\mathrm{Y}$ par $\mathrm{U}$ et $\mathrm{Z} \underset{\mathrm{p}_{2}}{\stackrel{\mathrm{p}_{1}}{\longrightarrow}} \mathrm{Y}$ par la relation induite sur U par Z (voir, par exemple, le lemme 1 de l'appendice I); cette relation induite sur $U$ est, elle-aussi, plate, quasi-finie et de présentation finie (comme le montre par exemple 3.1.2.2). On peut donc supposer dès le début que $y=p_{1} p_{2}^{-1}(y)$; donc $\mathrm{p}_{1}^{-1}(\mathrm{y})=\mathrm{p}_{2}^{-1}(\mathrm{y})$.

Ce dernier ensemble est contenu dans un ouvert affine de $Z$ (car $Z$ est alors quasi-affine). On peut donc "semi-localiser" $z$ suivant cet ensemble fini ; notons $z_{y}$ cet anneau semi-local.

Soient $\tilde{O}_{1}$ le hensélisé de I'anneau local $O_{\mathrm{Y}, \mathrm{y}}$ et $\tilde{O}_{2}$ le hensélisé de l'anneau semi-local $\mathrm{Z}_{\mathrm{y}}$. La donnée de la relation $\mathrm{Z} \underset{\mathrm{p}_{2}}{\stackrel{\mathrm{p}_{1}}{\longrightarrow}} \mathrm{Y}$ définit alors une relation d'équivalence finie et plate

$$
\operatorname{spec} \tilde{\mathrm{O}}_{2} \underset{\tilde{\mathrm{p}}_{2}}{\stackrel{\tilde{p}_{1}}{\longrightarrow}} \operatorname{Spec} \tilde{\mathrm{O}}_{1} \text {. }
$$

Rappelons que $\tilde{O}_{2}, \tilde{O}_{1}$ sont des anneaux henséliens (noethériens). Cette relation d'équivalence sur Spec $\tilde{O}_{1}$ admet alors un quotient $\operatorname{Spec} \tilde{O}$ (SGAD, V, 4.1) et I'on sait que $\tilde{O}_{1}$ est fini et plat sur $\tilde{O}$ (loc. cit.). L'anneau $\tilde{O}$ est donc un anneau local noethérien et hensélien.

$D^{\prime}$ autre part, le morphisme canonique $\operatorname{Spec} \tilde{\hat{O}}_{1} \rightarrow \mathrm{X}$ est compatible avec la relation $\left(\tilde{p}_{1}, \tilde{p}_{2}\right)$; puisque $X$ est un $S$-faisceau $f$ pqc on en déduit un S-morphisme $\tilde{\tau}: \operatorname{Spec} \tilde{O} \rightarrow X$.

Point a). Ce morphisme $\tilde{\tau}$ est formellement infinitésimalement étale au point fermé de $\operatorname{Spec} \tilde{0}$.

En effet, considérons le complété $\hat{O}_{1}\left(\operatorname{resp} . \hat{O}_{2}\right)$ de $\hat{O}_{\mathrm{y}} \quad\left(\operatorname{resp} . z_{\mathrm{y}}\right)$. Alors on a une relation d'équivalence finie et plate

$$
\operatorname{Spec} \hat{\mathrm{O}}_{2} \underset{\hat{\mathrm{p}}_{2}}{\stackrel{\hat{p}_{1}}{\longrightarrow}} \operatorname{spec} \hat{O}_{1}
$$

définie par $\left(p_{1}, p_{2}\right)$. Le quotient de $\operatorname{spec} \hat{O}_{1}$ par $\left(\hat{p}_{1}, \hat{p}_{2}\right)$ est alors de la forme spec $\hat{0}$, où $\hat{0}$ est un anneau local noethérien complet. Il est alors clair que $\hat{\theta}$ 
est le complété de $\tilde{O}$. D'autre part, il est formel de voir que $\hat{O}$ (avec l'élément canonique de $X(\hat{O}))$ promreprésente $X$ au point l'image de $y \cdot$ L'assertion est alors claire.

Point b). L'anneau local hensélien $\tilde{O}$ est le hensélisé d'un anneau local essentiellement de type fini sur $\mathrm{S}$.

Admettons ce point pour l'instant et complétons la démonstration de 3.1.1. Le faisceau $X$ est localement de type fini sur $S$ ([18], VI, 1.2.1) donc commute aux limites inductives filtrantes d'anneau sur $S$. On peut ainsi supposer qu'il existe un anneau local $\mathcal{O}$ essentiellement de type fini sur $S$ tel que $\tilde{O}$ soit le hensélisé de $\boldsymbol{O}$ et tel que $\tilde{\tau} \in \mathrm{X}(\tilde{\boldsymbol{O}})$ provienne d'un $\tau \in \mathrm{X}(\boldsymbol{O})$. Puisque la projection canonique $\operatorname{spec} \tilde{\mathcal{O}} \rightarrow \operatorname{spec} \mathcal{O}$ est formellement étale au point fermé de spec $\tilde{O}$, on conclut (du point a)) que $\tau$ est formellement infinitésimalement étale au point fermé de spec 0 .

Si $T$ est un schéma de type fini sur $\mathrm{S}$ tel que $\mathcal{O}$ soit le localisé de $\mathbb{O}_{\mathrm{T}}$ en un point $t \in T$, on peut supposer, quitte à restreindre $T$, que $\tau$ provient d'un S-morphisme $\alpha: T \rightarrow X$. Donc $\alpha$ est formellement infinitésimalement étale en $t$. Grâce à 3.1.2.3., on peut alors supposer, quitte à restreindre $T$, que $\alpha$ est étale (SGAD, XI). Ce qui achève la démonstration de 3.1 .1 modulo le point b); mais ce dernier est un cas spécial du résultat suivant.

3.1.2.5. Proposition : Soient $\Lambda$ un anneau noethérien, $\mathbf{A}_{1}, \mathbf{A}$ deux $\Lambda$-algèbres locales noethériennes et henséliennes, et $A \stackrel{\varphi}{\longrightarrow} A_{1}$ un $\Lambda$-morphisme local fini. Supposons que A est le hensélisé d'un anneau local essentiellement de type fini sur $\Lambda$; alors il en est de même de $A_{1}$. Et la réciproque est aussi vraie si $\varphi$ est injectif.

Démonstration : Par passage à la limite, l'assertion directe est claire. Prouvons donc la réciproque. L'anneau A est limite inductive filtrante de ses sous-1-algèbres $A(\alpha)$ de type fini. Pour tout $\alpha$ assez grand, il existe une

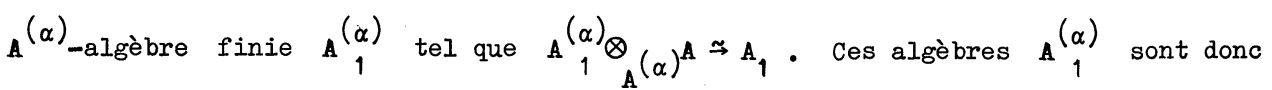


de type fini sur $\Lambda$ et on a $\underset{\alpha}{\lim } A_{1}^{(\alpha)}=A_{1}$. D'autre part, par hypothèse, on a un système filtrant $\{\stackrel{(i)}{1}\}_{i}$ de $\Lambda$-algèbres de type fini tel que les morphismes de transition de ce système soient étales de type fini, et que $\underset{i}{\lim } \mathbb{A} \underset{1}{(i)}=\mathbb{A}_{1}$.

On en conclut que pour un $i$ fixe, on a une factorisation

$\stackrel{(i)}{A_{1}} \rightarrow \mathbb{A}_{1}^{(\alpha)} \rightarrow \mathbb{A}_{1}^{(j)} \rightarrow \mathbb{A}_{1}$ pour $\alpha, j$ assez grand.

Soit pour chaque $\alpha$ (resp. i) $A^{\alpha}$ (resp. $A_{1}^{\alpha}, A_{1}^{i}$ ) le localisé de $A^{(\alpha)}$ (resp. $A_{1}^{(\alpha)}, A_{1}^{(i)}$ ) à l'idéal premier image inverse de l'idéal maximal de $\mathbf{A}$ (resp. $A_{1}$ ). On a alors les anneaux locaux $A^{\alpha}, A_{1}^{\alpha}, A_{1}^{i}$ essentiellement de type fini sur $\Lambda ;$ pour chaque $\alpha, \mathbb{A}_{1}^{\alpha}$ est essentiellement fini sur $\mathbb{A}^{\alpha}$. Et pour un i fixe, on a une factorisation $A_{1}^{i} \rightarrow A_{1}^{\alpha} \rightarrow A_{1}^{j}$ pour $\alpha, j$ assez grand ; on a également le fait que $A_{1}=\underset{\alpha}{\lim } A_{1}^{\alpha}=\underset{i}{\lim } A_{1}^{i}$ et que $A_{1}$ est le hensélisé de $A_{1}^{i}$ pour tout i .

Il en résulte que, dans la factorisation ci-dessus, $A_{1}^{\alpha} \rightarrow A_{1}^{j}$ est essentiellement quasi-fini. Fixons alors un tel $\alpha$; en passant aux hensélisés de $A_{1}^{\alpha}, A_{1}^{i}$ et $A^{\alpha}$ on obtient donc un diagramme commutatif

$$
\begin{aligned}
& \tilde{A}_{1}^{\alpha} \rightarrow A_{1} \\
& \hat{\Lambda}_{\tilde{A}}^{\alpha}
\end{aligned} \uparrow_{\varphi}
$$

où $\tilde{A}_{1}^{\alpha} \rightarrow \mathbb{A}_{1}, \tilde{A^{\alpha}} \rightarrow \tilde{A}_{1}^{\alpha}$ sont des morphismes finis. Donc $\tilde{A}^{\tilde{\alpha}} \rightarrow \mathbb{A}_{1}$ est fini ; puisque $A \stackrel{\varphi}{\longrightarrow} A_{1}$ est injectif, on conclut que $\tilde{A}^{\alpha} \rightarrow A$ est $\underline{\text { fini. }}$. D'où on termine à l'aide du cas direct de la proposition.

C.Q.F.D.

\subsection{Une "descente" entière d'espaces algébriques}

3.2.0. Dans ce qui suit, nous adoptons la convention suivante : Soient $\mathbf{S}$ un schéma et $\mathrm{P}$ une propriété de S-schémas (resp. de morphismes de S-schémas) qui est "de nature locale pour la topologie étale" ; alors on peut parler d' un S-espace algébrique (resp. d'un morphisme de S-espaces algébriques) qui possède la propriété $P$. Citons entre autres, la propriété d'être réduit, normal,... (resp. d'être 
un morphisme affine, entier, fini,...). On peut aussi parler d'un faisceau quasicohérent en modules (resp. algèbres) sur un s-espace algébrique ; en particulier, à toute Algèbre quasi-cohérente $t$ sur un S-espace algébrique $X$, on peut associer un s-espace algébrique, affine sur $X$, noté spec t.

Pour les définitions précises de ces notions et pour les fondations de la théorie des Espaces Algébriques, nous renvoyons le lecteur à [13]. Signalons toutefois que, pour un morphisme d'un S-schéma $\tilde{X}$ dans un S-espace algébrique $X$, les justifications nécessaires résultent directement de 3.1.2.1.

Le but principal de cette section est d'établir le résultat technique suivant.

3.2.1. Théorème : Soient $\mathrm{S}$ un schéma localement noethérien, $\mathrm{X}$ un S-espace algébrique (loc. PF) et séparé $(\mathrm{cf} .1 .3 .0$ ) et $\tilde{X}$ un S-schéma. Supposons que I'on a S-morphisme $\pi: \tilde{X} \rightarrow X$ entier et surjectif dont chaque fibre est contenue dans un ouvert affine de $\tilde{X}$. Alors $X$ est un S-schéma.

Remarquons tout de suite que I'on peut supposer $S$ affine et $\tilde{X}$ réduit ; ensuite on peut également supposer $X$ noethérien et réduit (cf. [7], III, n०2, I $_{7}$ ).

\subsubsection{Une réduction préliminaire}

Avec les notations de 1.3 .0$. , on représente $X$ comme le conoyau $\mathrm{Z} \stackrel{\mathrm{p}_{1}}{\longrightarrow} \mathrm{Y} \stackrel{\mathrm{p}}{\longrightarrow} \mathrm{X}$. D'après le théorème 6 de l'appendice $I$, la question de la repré$\mathrm{p}_{2}$ sentabilité de $X$ se ramène à montrer que l'espace annelé quotient de $Y$ par $Z$ est un S-schéma; et d'après le théorème 5 de l'appendice $I$, ce dernier problème "se teste" sur les anneaux locaux de $Y$ et les relations induites. Notre méthode de démonstration suivra cette ligne, surtout dans le cas où $X$ est non normal. Dans ce numéro donc notre but sera d'obtenir quelques renseignements "utiles" sur la relation d'équivalence induite par $\mathrm{Z} \underset{\mathrm{p}_{2}}{\stackrel{\mathrm{p}_{1}}{\Longrightarrow}} \mathrm{Y}$ sur les anneaux locaux de $\mathrm{Y}$. Notons d'abord que la suite $\stackrel{2}{\stackrel{p_{1}}{p_{2}}} Y \stackrel{p}{\longrightarrow} X$ donne, par le changement de base $\pi: \tilde{X} \rightarrow X$, une situation du type suivant : 


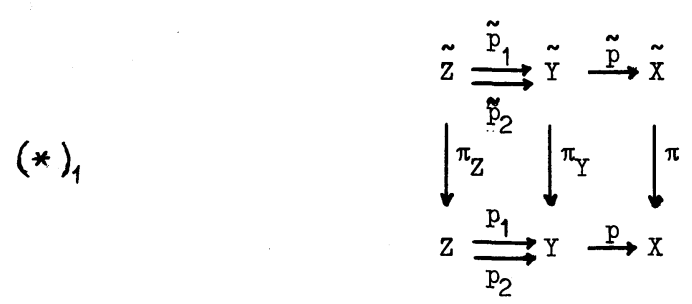

où tous les carrés sont cartésiens, et $\tilde{Y}=Y \times{ }_{X} \tilde{X}, \tilde{Z}=Z \times \frac{\tilde{X}}{X}$ sont des S-schémas (cf. 3.1.2.1); les morphismes $\pi_{Y}, \pi_{Z}$ sont donc des morphismes entiers et surjectifs de S-schémas.

Soit $y$ un point quelconque de $\mathrm{Y}$. Nous allons déduire une situation, analogue à $(*)_{1}$, où $Y$ sera remplacé par un schéma semi-local (sous-objet de $Y$, et) contenant le point $\mathrm{y}$, et les relations seront remplacées par les relations induites.

Pour réaliser cette réduction nous ferons, dans 3.2.1., I'hypothèse supplémentaire suivante :

(F) : Les fibres de $\pi$ n'ont qu'un nombre fini de points.

Rappelons que l'on a pu supposer $X$ noethérien. Suivant le procédé de 1.3.1 a), on peut donc supposer $Y$ affine au sens absolu. Puisque $S$ est supposé affine et $X$ séparé, $Z$ est aussi affine et par suite $\tilde{Y}, \tilde{Z}$ sont affines. D'autre part, $\tilde{X} \times \frac{\tilde{X}}{X}$ est lui-aussi un schéma (cf. 3.1.2.4) tel que les projections canoniques $\tilde{\mathrm{X}} \times \tilde{\mathrm{X}} \underset{\mathrm{pr}_{2}}{\stackrel{\mathrm{pr}_{1}}{\Longrightarrow}} \tilde{\mathrm{X}}$ soient des morphismes entiers et surjectifs. On trouve alors le diagramme suivant à trois étages :

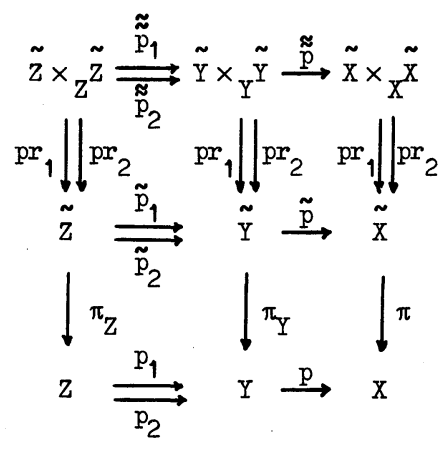


où tous les carrés sont cartésiens, tous les faisceaux sauf $X$ sont des schémas, les suites horizontales (strictes) exactes et les morphismes verticaux entiers et surjectifs.

Soit $x$ l'image (ensembliste) dans $X$ du point $y \in Y$ choisi plus haut. La fibre $\pi^{-1}(x)$ est un ensemble fini dans $\tilde{X}$ (d'après $(F)$ ), contenu dans un ouvert affine de $\tilde{X}$ par hypothèse. Il existe donc un schéma semi-local $\tilde{E}$ et un monomorphisme plat quasi-compact $\tilde{e}: \tilde{E} \hookrightarrow \tilde{X}$ dont l'image est l'ensemble des générisations des points de $\pi^{-1}(\mathrm{x})$. Or, l'ensemble $\tilde{\mathrm{p}}^{-1}\left(\pi^{-1}(\mathrm{x})\right)$ est une partie finie du schéma affine $\tilde{Y}$, donc on trouve un schéma affine semi-local $\tilde{F}$ et un monomorphisme plat quasi-compact $\tilde{f}: \tilde{F} \leftrightarrow \tilde{Y}$ dont l'image est l'ensemble des générisations des points de $\tilde{p}^{-1}\left(\pi^{-1}(x)\right)$. Puisque le morphisme $\tilde{p}$ est $f$ ppf on trouve un morphisme fpqc $\tilde{F} \stackrel{\tilde{\theta}}{\longrightarrow} \tilde{E}$ tel que le diagramme

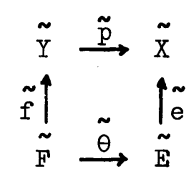

soit commutatif. Comme $\tilde{\mathrm{Z}} \cong \tilde{\mathrm{Y}} \times{ }_{\tilde{\mathrm{X}}} \tilde{\mathrm{Y}}$, la relation induite par $\tilde{\mathrm{Z}} \rightarrow \tilde{\mathrm{Y}}$ sur $\tilde{\mathrm{F}}$ (pour le morphisme $\tilde{f})$ n'est rien d'autre que la relation d'équivalence

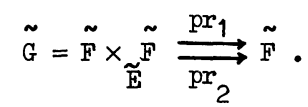

Donc la suite $\tilde{\mathrm{G}} \Longrightarrow \tilde{\mathrm{F}} \stackrel{\tilde{\theta}}{\longrightarrow} \tilde{\mathrm{E}}$ est exacte; $\tilde{\mathrm{E}}, \tilde{\mathrm{F}}$ sont des schémas affines semilocaux, et $\tilde{G}$ est affine.

Considérons dans $\tilde{Y} \times{ }_{Y} \tilde{Y}$ les deux sous-objets $\operatorname{pr}_{1}^{-1}(\tilde{F})$ et $\operatorname{pr}_{2}^{-1}(\tilde{F})$; je dis qu'ils coincident. En effet, $\mathrm{pr}_{i}, i=1,2$, est entier, donc fermé, et on en déduit que l'image du monomorphisme plat quasi-compact $\operatorname{pr}_{i}^{-1}(\tilde{f}): \operatorname{pr}_{i}^{-1}(\tilde{F}) \rightarrow \tilde{Y} \times \tilde{Y}_{Y}$ est l'ensemble des générisations des points de $\operatorname{pr}_{i}^{-1}\left(\tilde{p}^{-1}\left(\pi^{-1}(x)\right)\right)$. Il suffit alors de remarquer que :

i) $\operatorname{pr}_{1}^{-1}\left(\tilde{p}^{-1}\left(\pi^{-1}(\mathrm{x})\right)\right)=\operatorname{pr}_{2}^{-1}\left(\tilde{p}^{-1}\left(\pi^{-1}(\mathrm{x})\right)\right)$

ii) un monomorphisme plat quasi-compact dans un schéma est entièrement 
déterminé par son image dans ce schéma (cf. [21], 1.3).

Il s'ensuit qu'il existe uil schéma affine semi-local $F$ et un monomorphisme plat quasi-compact $f: F \leftrightarrow Y$ tel que $(\tilde{F}, \tilde{f})=\pi_{Y}^{-1}(F, f)$ (loc. cit., 3.4). Et l'image de $f$ est l'ensemble des générisations des points de $p^{-1}(x)=p_{1} p_{2}^{-1}(y)$. Soit $G \rightrightarrows F$ la relation induite par $Z \rightrightarrows Y$ sur $F$ (pour le morphisme $f$ ). on a alors un diagramme

$(*)_{2}$

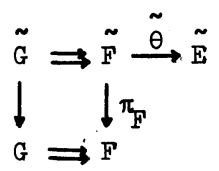

où la suite supérieure est exacte. Il est formel de voir que les deux carrés à gauche sont cartésiens, de sorte que $\pi_{F}, \pi_{G}$ sont des morphismes entiers et surjectifs. D'où on déduit que G est affine.

Rappelons que $F$ est un sous-objet plat semi-local de $Y$ "contenant" $y$ et que $\mathrm{y}$ est un point quelconque de $\mathrm{Y}$. Nous nous servirons de cette "réduction 10cale" dans le cas où $\pi$ est $\underline{\text { fini }}$, et où donc l'hypothèse supplémentaire ( $F$ ) sur les fibres de $\pi$ sera vérifiée.

3.2.3. Le cas affine de 3.2 .1

3.2.3.1. Proposition : Avec les hypothèses de 3.2.1., supposons de plus que $\tilde{X}$ est un schéma affine au sens absolu. Alors $X$ est un schéma affine.

Grâce à [21] 3.1., il s'agit simplement de montrer que $X$ est un schéma. On peut donc supposer $S$ affine, $\tilde{X}$ réduit, et ensuite $X$ noethérien, réduit. Par un raisonnement du passage à la limite (EGA, IV, 8) on se ramène ensuite à supposer que S (resp. Y) est de type fini sur $\operatorname{Spec} Z$, en utilisant le

3.2.3.2. Lemme : On peut supposer dans 3.2.3.1 que $\pi: \tilde{X} \rightarrow X$ est fini, surjectif.

En effet, le faisceau $X$ commute aux limites inductives filtrantes d'anneau sur $S$ (cf. [18], VI, 1.2.1). Comme $\tilde{A}=\mathbf{A}(\tilde{X})$ est limite inductive filtrante de ses sous-anneaux $\mathbb{A}_{i}$ de type fini sur $S, \pi \in X(\tilde{A})$ provient d'un $\pi_{i} \in X\left(\mathbb{A}_{i}\right)$, 
pour $i$ assez grand. On peut donc remplacer $\tilde{X} \operatorname{par}$ Spec $A_{i}$ et $\pi \operatorname{par} \pi_{i}$, pour un $i$ assez grand.

C.Q.F.D.

Considérons alors le diagramme suivant (comme dans 3.2.2):

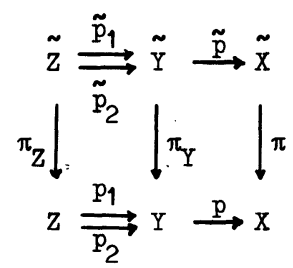

où $Z, Y, \tilde{Y}, \tilde{X}, \tilde{Z}$ sont des schémas sur $S$, affines au sens absolu, et $\pi_{Y}, \pi_{Z}$ sont des morphismes finis et surjectifs. En posant donc $Y=\operatorname{Spec} B, \tilde{Y}=\operatorname{Spec} \tilde{B}$, $\mathrm{Z}=\operatorname{Spec} \mathrm{C}, \tilde{\mathrm{Z}}=\operatorname{Spec} \tilde{\mathrm{C}}$ et $\tilde{\mathrm{X}}=\operatorname{Spec} \tilde{\mathrm{A}}$, on trouve le diagramme

$$
\begin{aligned}
& \tilde{\mathrm{A}} \stackrel{\tilde{\delta}}{\longrightarrow} \tilde{\mathrm{B}} \stackrel{\tilde{\delta}_{1}}{\longrightarrow} \tilde{\mathrm{C}} \\
& i_{B} \uparrow \tilde{\delta}_{2} \uparrow_{i_{C}} \quad \delta_{i}={ }^{a} p_{i}, \tilde{\delta}_{i}={ }^{a} \tilde{p}_{i}, i=1,2, \\
& A \rightarrow B \underset{\delta_{2}}{\stackrel{\delta_{1}}{\longrightarrow}} \mathrm{C} \quad \tilde{\delta}={ }^{\mathrm{a}} \tilde{\mathrm{p}}, \ldots .
\end{aligned}
$$

où A est défini comme le sous-anneau d'égalité de $B \underset{\delta_{2}}{\stackrel{\delta_{1}}{\longrightarrow}} \mathrm{C}$, la suite supérieure est stricte exacte et les deux carrés à droite sont co-cartésiens. D'après les théorèmes 5, 6 de l'appendice I, pour prouver la représentabilité de $X$, il suffit de prouver que $\tilde{\boldsymbol{A}}$ est entier sur $\mathbf{A}$.

Point a). Le normalisé de $X$ est un schéma (affine).

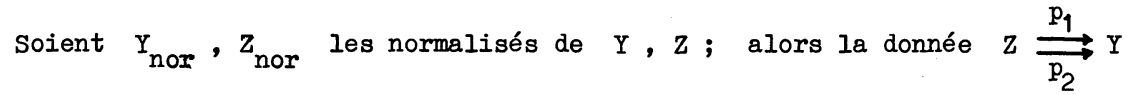
définit une relation d'équivalence étale, de présentation finie $\mathrm{Z}_{\text {nor }} \rightarrow \mathrm{Y}_{\text {nor }}$ et le normalisé $X_{\text {nor }}$ de $X$ est le faisceau fppf quotient de cette dernière relation. Par ailleurs, Y, Z étant de type fini sur Spec Z, Y ${ }_{\text {nor }}, Z_{\text {nor }}$ le sont également; donc $X_{n o r}$ est un S-espace algébrique (loc. PF) et séparé, fini sur $X$. Quitte à remplacer $X, Y, Z, \tilde{X}$ plus haut par leurs normalisés, on peut les supposer normaux. 
On doit montrer que $X=X_{\text {nor }}$ est un schéma; on peut donc remplacer $Y$ par une composante irréductible $(\mathrm{z} \rightarrow \mathrm{Y}$ par la relation induite $)$ et $\mathrm{X}$ par l'ouvert correspondant; $\mathrm{Y}$ est alors intègre. Dans le diagramme $(*)$ plus haut, $\tilde{B}$ est donc un B-module sans torsion. Par suite, pour un $\tilde{a} \in \tilde{A}$ quelconque, on peut définir le polynôme caractéristique $P\left(i_{B}, \tilde{\delta}(\tilde{a})\right)$ de $\tilde{\delta}(\tilde{a}) \in \tilde{B}$ sur $B$.

Puisque les deux carrés à droite (dans $(*)$ ) sont cocartésiens et que $\tilde{\delta}_{2} \tilde{\delta}=\tilde{\delta}_{1} \tilde{\delta}$, on a l'égalité

$$
\delta_{1} P\left(i_{B}, \tilde{\delta}(\tilde{a})\right)=P\left(i_{C}, \tilde{\delta} \tilde{\delta}_{1}(\tilde{a})\right)=P\left(i_{C}, \tilde{\delta}_{2} \tilde{\delta}(\tilde{a})\right)=\delta_{2} P\left(i_{B}, \tilde{\delta}(\tilde{a})\right) ;
$$

ce qui veut dire que les coefficients de $P\left(i_{B}, \tilde{\delta}(\tilde{a})\right)$ sont des éléments de A Par Caylay-Hamilton on a alors $P\left(i_{B}, \tilde{\delta}(\tilde{a})\right)(\tilde{\delta}(\tilde{a}))=0 ;$ comme $\tilde{\delta}$ est injectif, cela montre que ã est entier sur A.

Point b). $X$ est un schéma.

Grâce au point a) et au lemme 3.2.3.2 on peut supposer que le morphisme $\pi: \tilde{X} \rightarrow X$ est birationnel, fini. L'hypothèse supplémentaire ( $F$ ) de 3.2 .2 est donc vérifiée ; en faisant recours à la "réduction locale" de 3.2.2., on peut supposer que le diagramme $(*)$ plus haut est le diagramme d'anneaux correspondant à la situation ( semi)-locale $(*)_{2}$ de 3.2 .2 .

Pour montrer que le plus grand ouvert de $Y$, au-dessous duquel $X$ est un schéma, contient les points de $F=\operatorname{Spec} B$, il suffit toujours de montrer que $\tilde{A}$ est entier sur A (Appendice I, théorèmes 5, 6).

Puisque $\tilde{B}$ est fini, birationnel sur B, le conducteur $I$ de $\tilde{B}$ dans $B$ est $\neq(0)$; c'est un idéal de $B$ (resp. $\tilde{B}$ ) stable par la relation d'équivalence $\mathrm{B} \rightarrow \mathrm{C} \quad(\operatorname{resp} . \tilde{\mathrm{B}} \rightarrow \tilde{\mathrm{C}})$ de sorte que la réduction mod I nous fournit un second diagramme

$(\bar{*})$

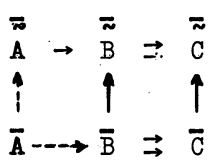


où $\bar{A}$ est défini comme le sous-anneau d'égalité de $\bar{B} \rightrightarrows \bar{C}$, la suite supérieure est stricte exacte, et les deux carrés à droite sont co-cartésiens.

Pour montrer que Á est entier sur A, nous raisonnons par récurrence sur dim B ("relativement à l'existence d'un tel diagramme"). Si dim B=0, il n'y a rien à démontrer, car $\tilde{\mathrm{Y}} \rightarrow \mathrm{Y}$ est birationnel fini.

Soit $\tilde{\alpha} \in \tilde{A}$ un élément quelconque. Par l'hypothèse de récurrence, son image $\overline{\tilde{\alpha}}$ dans $\overline{\tilde{A}}$ est entière sur $\bar{A} ;$ soit alors $\overline{\tilde{\alpha}} n+\sum_{0 \leqslant i \leqslant n-1} \bar{a}_{i} \overline{\bar{\alpha}}^{i}=0$ une équation de dépendence intégrale, à coefficients $\bar{a}_{i}$ dans $\bar{A}$. Pour chaque $i, 0 \leqslant i \leqslant n-1$, on choisit un relèvement $a_{i}$ dans $\tilde{A}$ de l'image de $\bar{a}_{i}$ dans $\overline{\tilde{A}}$. On a alors le

3.2.3.3. Lemme : Pour tout $i, 0 \leqslant i \leqslant n-1$, l'élément $a_{i}$ de $\tilde{A}$, considéré comme élément de $\tilde{B}$, est dans l'image de $B \stackrel{i_{B}}{\longrightarrow} \tilde{B}$.

En effet, on peut trouver un élément $b_{i}^{\prime} \in B$ tel que son image $\bar{b}_{i}^{\prime}$ dans $\bar{B}$ s'envoie sur l'image de $a_{i}$ dans $\overline{\tilde{B}}$. Ce qui signifie que, dans $\tilde{B},\left(a_{i}-b_{i}^{\prime}\right)$ est un élément de I. $\tilde{B} \hookrightarrow B$; i.e., $\left(a_{i}-b_{i}^{\prime}\right)$ est dans l'image de $B \stackrel{{ }^{i} B}{\longrightarrow} \tilde{B}$, et $i l$ en est donc de même de $a_{i}$.

Rappelons que l'on a pu supposer X réduit, donc aussi $Y, Z$. D'où il résulte que $i_{C}: C \rightarrow \tilde{C}$ est injectif. Les $a_{i}, \circ \leqslant i \leqslant n-1$, proviennent donc de $A$. Considérons alors l'élément $\tilde{a}=\tilde{\alpha}^{n}+\sum_{0 \leqslant i \leqslant n-1} a_{i} \tilde{\alpha}^{i}$ de $\tilde{A} ;$ pour voir que $\tilde{\alpha}$ est entier sur A, il suffit de montrer que ã est un élément de A. Mais puisque son image dans $\tilde{\tilde{A}}$ est zéro, on a $\tilde{a} \in I . \tilde{B} \hookrightarrow B$; donc $\tilde{a} \in \tilde{A} \cap B=A$. C.Q.F.D.

3.2.4. Le cas général de 3.2.1

a) Réduction au cas $\pi \underline{\text { fini }}$

Considérons le morphisme affine $\pi: \tilde{X} \rightarrow X$. Puisque $X$ est noethérien, on sait que, d'après $[13], \sqrt{15.155)}, \pi(\tilde{x}, \pi)$ est limite projective filtrante d'un système $\left(x_{i}, \pi_{i}\right)_{i \in I}$ de S-espaces algébriques de type fini et affines sur $X$. or, soient $x$ un point quelconque de $X$ et $\tilde{V}$ un ouvert affine de $\tilde{X}$ contenant la fibre $\pi^{-1}(x)$. Alors, il existe un ouvert quasi-compact $v_{i}$ d'un $x_{i}$ tel que $\tilde{V} \simeq v_{i} x_{i} \tilde{X}$ : en effet, considérons les espaces topologiques sous-jacents 
$\operatorname{Top}\left(x_{i}\right)$ des S-faisceaux $x_{i}\left({ }^{*}\right) ; a l o r s$ on a aussi $\operatorname{Top}(\tilde{X})=\underset{i \in I}{\lim } \operatorname{Top}\left(x_{i}\right)$ et I'assertion est donc claire.

Si $\varphi_{i}$ est la projection canonique $\tilde{X} \rightarrow X_{i}, \varphi_{i} \mid \tilde{V}: \tilde{V} \rightarrow v_{i}$ est entier, surjectif. Puisque $\tilde{V}$ est un schéma affine, il résulte du cas affine 3.2 .3 que $v_{i}$ est un schéma, affine au sens absolu.

D'autre part, considérons les projections canoniques $\tilde{\mathrm{X}} \times \frac{\tilde{\mathrm{X}}}{\stackrel{\mathrm{pr}_{1}}{\longrightarrow}} \tilde{\mathrm{pr}}$; alors $\left(\tilde{\mathrm{V}}-\mathrm{pr}_{1} \operatorname{pr}_{2}^{-1}(\tilde{\mathrm{X}}-\tilde{\mathrm{V}})\right)=\tilde{\mathrm{W}}$ est un ouvert de $\tilde{\mathrm{V}}$ tel que $\operatorname{pr}_{1}^{-1}(\tilde{\mathrm{W}})=\operatorname{pr}_{2}^{-1}(\tilde{\mathrm{W}})$ et $\tilde{\mathrm{W}}$ contient la fibre $\pi^{-1}(x)$. Il s'ensuit que $\tilde{W}=\pi^{-1}(U)$ pour un ouvert $U$ de $X$ contenant $x$. Si $U_{i}$ est l'ouvert $\pi_{i}^{-1}(U)$ de $x_{i}$, alors $U_{i}$ est un ouvert de $v_{i}$ donc est un schéma; d'autre part $U \hookrightarrow X$ est quasi-compact et il en est donc de même de $U_{i} \hookrightarrow V_{i} ;$ d'où $U_{i}$ est un schéma quasi-affine. Quitte à remplacer $X$ par $U, \tilde{X}$ par $U_{i}$ et $\pi$ par $\pi_{i} \mid U_{i}$, on peut alors supposer $\tilde{X}$ quasi-affine (au sens absolu) et $\pi$ fini.

b) Fin de la démonstration

Puisque $\pi$ est fini, la réduction faite dans 3.2.2 peut-être appliquée. Avec les notations de 3.2.2., pour un point $y$ quelconque de $Y$, on trouve ainsi un diagramme d'anneaux du type $\left(_{*}\right)$ de 3.2.3., tel que Spec B soit un sous-objet plat semi-local de $\mathrm{Y}$ contenant $\mathrm{y}$. Avec les notations de ce même diagramme $(*)$, on prouve alors que $\tilde{\mathbf{A}}$ est entier sur $\mathbf{A}$, suivant le même argument que dans le cas 3.2.3. On termine (comme avant) par un appel auxthéorèmes 5,6 de I'appendice I. C.Q.F.D.

3.2.5. Remarque : Nous avons en fait démontré le résultat suivant : Soient $S$ un schéma localement noethérien, $X$ un s-espace algébrique (loc. PF) séparé, $\tilde{X}$ un S-espace algébrique quelconque, $\pi: \tilde{X} \rightarrow X$ un morphisme entier et $x$ un point de $X$. Supposons que " $\tilde{X}$ est un schéma affine au voisinage de la fibre $\pi^{-1}(x)$ ". Alors $X$ est un schéma au voisinage du point $x$.

$\left(^{*}\right)$ af. $[13]$, p. 132 . 
3.3. Une première application : Existence du quotient en codim 1

Soient $\mathrm{S}$ un schéma localement noethérien, $\mathrm{Z}, \mathrm{Y}$ des S-schémas localement

de type fini, $Z \underset{p_{2}}{\stackrel{p_{1}}{\longrightarrow}} Y$ une s-relation d'équivalence plate et de type fini, et $X$ le S-faisceau fppf quotient. Alors on a vu (cf. 3.1.1) que $X$ est un S-espace algébrique (loc. PF); cela nous permet de parler de la codimension d'un point $\mathbf{x}$ dans $X$.

3.3.1. Proposition : Avec les notations ci-dessus, supposons de plus que $X$ est séparé sur $S$ (i.e., le morphisme canonique $d: Z \stackrel{\left(p_{1}, p_{2}\right)}{\longrightarrow} Y x_{S} Y$ est une immersion fermée). Alors le plus grand ouvert $U$ de $X$ qui est un schéma contient les points de codimension $\leqslant 1$ de $X$.

Démonstration : On peut supposer ci-dessus que $Z \underset{\mathrm{p}_{2}}{\stackrel{\mathrm{p}_{1}}{\longrightarrow}} \mathrm{Y}$ est une relation d'équivalence étale, de type fini. Soient $y$ un point de codimension 1 de $Y$ et $\mathbf{x}$ son image dans $X$. Pour montrer que $X$ est un schéma au voisinage de $\mathbf{x}$, on peut supposer $X$ (et donc $Y, Z$ ) réduit (cf. [7], III, No2, \$7), et noethérien. Soit $\tilde{Y}$ (resp. $\tilde{Z}$ ). le normalisé de $Y$ (resp. Z). La donnée de la relation $\mathrm{Z} \underset{\mathrm{p}_{2}}{\stackrel{\mathrm{p}_{1}}{\longrightarrow}} \mathrm{Y}$ définit alors une relation d'équivalence étale et de présentation finie $\tilde{Z} \underset{\tilde{p}_{2}}{\stackrel{\tilde{p}_{1}}{\longrightarrow}} \tilde{Y}$. Le normalisé $\tilde{X}$ de $X$ est le s-faisceau fppf quotient de cette dernière relation. Si $\tilde{\pi}: \tilde{X} \rightarrow X$ est la projection canonique, alors, d'après $[13], p .155$, $(\tilde{x}, \tilde{\pi})$ est limite projective d'un système filtrant $\left(x_{i}, \pi_{i}\right)_{i \in I}$ de S-espaces algébriques finis sur $X$.

Nous allons montrer qu'il existe $i_{0} \in I$ assez grand tel que $x_{i}$ soit "un schéma affine au voisinage de la fibre $\pi_{i}^{-1}(x) "$. Grâce à 3.2.5., cela prouvera 3.3 .1

Considérons l'anneau local $\mathbf{A}=\mathrm{O}_{\mathrm{Y}, \mathrm{y}}$. La relation induite par $\mathrm{Z} \underset{\mathrm{p}_{2}}{\stackrel{\mathrm{p}_{1}}{\longrightarrow}} \mathrm{Y}$ sur Spec $A$ est alors de la forme $\operatorname{spec} B \underset{p_{2}}{\stackrel{p_{1}}{\longrightarrow}}$ Spec $A$ et c'est une relation d'équivalence étale. I'anneau $B$ est alors semi-local, noethérien, réduit, de dimension 1. Soit $\tilde{A}$ (resp. B) le normalisé de A (resp. B); on a alors la relation d'équi- 
valence étale "déduite" Spec $\tilde{B} \underset{\tilde{p}_{2}}{\stackrel{\tilde{p}_{1}}{\longrightarrow}} \operatorname{Spec} \tilde{A}$.

Point 1). Cette dernière relation admet un conoyau dans ( $\mathrm{Sch} / \mathrm{S})$.

Remarquons que Spec $\tilde{A}$ est un semi-trait. Pour prouver ce point, on peut donc supposer que Spec $\tilde{A}$ est un trait (lemme 1, appendice I). L'anneau $\tilde{A}$ est alors un anneau de valuation discrète. Si $K$ est son corps des fractions, la relation induite sur Spec K est finie étale, donc admet un conoyau de la forme Spec I, où $L$ est un sous-corps de $K$ tel que $K$ soit une extension finie séparable de $I$. Soit $A_{1}$ I'anneau de valuation discrète $A \cap L$. On a que spec $\tilde{B}$ est un fermé de (Spec $\left.\tilde{A} \times{ }_{S} \operatorname{Spec} \tilde{A}\right)$ qui coIncide avec (Spec $\left.\tilde{A} \times{ }_{\text {Spec } A_{1}} \operatorname{Spec} \tilde{A}\right)$ sur l'ouvert (Spec $K \times_{S}$ Spec $\left.K\right)$. D'où on conclut que Spec $\tilde{\sim}^{\prime}$ est égal à $\left(\operatorname{Spec} \tilde{A} \times\right.$ Spec $A_{1}$ Spec $\left.\tilde{A}\right)$. D'autre part, Spec A est plat sur Spec $A_{1}$; il en résulte que Spec $A_{1}$ est le conoyau (faisceautique) de $\operatorname{spec} \tilde{B} \underset{\tilde{\tilde{p}_{2}}}{\stackrel{\tilde{p}_{1}}{\longrightarrow}} \operatorname{spec} \tilde{A}$. Point 2). Le conoyau de $\operatorname{spec} \tilde{\mathrm{B}} \underset{\tilde{\mathrm{p}}_{2}}{\stackrel{\tilde{p}_{1}}{\longrightarrow}}$ Spec $\tilde{\mathrm{A}}$ est un schéma affine $\tilde{\mathrm{E}}$.

Cela résulte du fait que ce conoyau est un schéma noethérien (normal), séparé, de dimension 1 à ensemble sous-jacent fini, et du lemme suivant.

3.3.1.1. Lemme (cf. [18], VIII.1) : Soit $\mathrm{T}$ un schéma localement noethérien, séparé. Alors chaque ensemble fini de points de codimension $\leqslant 1$ dans $T$ est contenu dans un ouvert affine de $\mathrm{T}$.

Point 3). Si le normalisé $\tilde{X}$ de $X$ est un S-espace algébrique (10c. PF) (par exemple, si $S$ est de type fini sur Spec $\mathbf{z}$ ), alors les faits précédents montrent que $\tilde{X}$ est un schéma (affine) au voisinage de la fibre $\pi^{-1}(x)$ (cf. théorèmes 5,6 de I'appendice I). Dans ce cas, grâce à 3.2.5., la démonstration de 3.3 .1 s'achèvera ici.

Point 4). Dans le cas où $\tilde{X}$ n'est pas de type fini sur $x$, nous procédons comme suit.

Notons $\mathrm{A}_{0}, \mathrm{~B}_{0}, \tilde{\mathrm{A}}_{0}, \tilde{\mathrm{B}}_{0}$ les réductions de $\mathrm{A}, \mathrm{B}, \tilde{\mathrm{A}}, \tilde{\mathrm{B}}$ modulo l'idéal maximal $m$ de $A=y_{y, y}$. Donc on a la relation étale finie "déduite" 
Spec $\tilde{B}_{0} \rightarrow$ Spec $\tilde{A}_{0}$ sur le schéma artinien spec $\tilde{A}_{0} ;$ son conoyau est un schéma artinien $\tilde{E}_{0}$, dont les corps résiduels sont des extensions de type fini sur $k(s)$, où $s$ est la projection de $y$ dans $s$ (Krull-Akizuki). Puisque $\tilde{E}$ est affine (Point 2)), on peut relever des générateurs de ces $k(s)$-extensions en un nombre fini d'éléments de $\Gamma(\tilde{E}) ;$ soient $a_{\alpha}, \alpha=1, \ldots r$, les images de ces relèvements $\operatorname{dans} \tilde{\mathrm{A}}$.

Remarquons que I'on peut supposer $Y, Z, \tilde{Y}, \tilde{Z}$ affines; quitte à restreindre $Y$ et à modifier les $\left\{a_{\alpha}\right\}$ par des éléments de $\Gamma(Y)$, on peut supposer que ces $a_{\alpha}$ proviennent de $\Gamma(\tilde{Y})$ et en fait de $\operatorname{ker}(\Gamma(\tilde{Y}) \underset{\rightarrow}{\rightarrow}(\tilde{z}))$. Ecrivant $(\tilde{X}, \pi)=\underset{i \in I}{\lim }\left(X_{i}, \pi_{i}\right)$, posons par suite $Y_{i}=Y x_{X} X_{i}, Z_{i}=Z x_{X} X_{i}, i \in I$. Alors $Y_{i}\left(\right.$ resp. $\left.Z_{i}\right)$ est un schéma fini sur $Y$ (resp. $\left.Z\right)(c f \cdot[13], p .138)$; et on a $\underset{i \in I}{\lim } \Gamma\left(Y_{i}\right)=\Gamma(\tilde{Y}) ; \underset{i \in I}{\lim } \Gamma\left(z_{i}\right)=\Gamma(\tilde{Z})$. Il existe donc $i_{0} \in I$ assez grand tel que les a proviennent de $\Gamma\left(Y_{i_{0}}\right)$ et en fait de $\operatorname{ker}\left(\Gamma\left(Y_{i_{0}}\right) \rightarrow \Gamma\left(z_{i_{0}}\right)\right)=C_{i_{0}}$. Il est alors immédiat que le morphisme canonique $\mathrm{Y}_{i_{0}} \stackrel{\varphi}{\rightarrow}$ Spec $\mathrm{C}_{i_{0}}$ est compatible avec

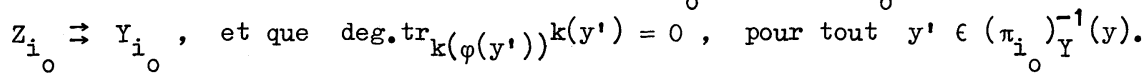
D'après les théorèmes 5,6 de l'appendice $I, X_{i_{0}}$ est un schéma au voisinage de la fibre $\pi_{i}^{-1}(x)$. On termine par 3.3.1.1 et 3.2 .5 . C.Q.F.D. 3.3.2. Remarque : La proposition 3.3 .1 est fausse si l'on ne fait pas l'hypothèse de séparation sur $X$, ou si l'on considère les points de codimension $\geqslant 2$ de $X$. En effet on a un contre-exemple d'un espace algébrique $G$ en groupes (Ioc. PF) (resp. (1oc. PF) et séparé) et lisse sur un trait (resp. sur un anneau local régulier de dimension 2) tel que le plus grand ouvert représentable de G ne rencontre pas la fibre de $G$ au-dessus du point fermé de la base, cf. SGAD, VI ${ }_{B}$ page 81 (resp. [18], X.14). 
3.4. Application aux espaces homogènes

3.4.1. Proposition : Soient $S$ un schéma de Dedekind et $G$ un S-espace algébrique en groupes, (loc. PF), séparé, et plat sur $\mathrm{S}$. Alors il existe un schéma $\mathrm{S}_{1}$ fpqc sur $S$ tel que $G_{S_{1}}$ soit un schéma. Plus précisément, si $\mathbb{K}$ est le corps des fractions de $S$ et $\overline{\mathrm{K}}$ une clôture algébrique de $K$, le normalisé $S_{1}$ de $S$ dans $\overline{\mathrm{K}}$ est tel que $G_{S_{1}}$ soit un schéma.

Démonstration : On peut supposer que $s$ est un trait $(s, \eta, s)$. Représentons $G$ comme le conoyau $Z \underset{p_{2}}{\stackrel{p_{1}}{\longrightarrow}} Y \stackrel{p}{\longrightarrow} G$, comme dans 1.3 .0 ; le schéma $Y$ est donc plat et localement de type fini sur $S$. Si $y$ est un point fermé de $Y_{S}$, alors on voit comme dans EGA, IV, 17.16.1 qu'il existe une s-quasi-section plate de Y passant par $\mathrm{y}$. Cette quasi-section devient nécessairement localement triviale sur $\mathrm{S}_{1}$.

Par ailleurs, d'après 3.3.1., le plus grand ouvert $U$ de $G$ qui est un S-schéma contient $\left(G_{\eta}\right.$ et) les points maximaux de $G_{s}$ (i.e., images des points maximaux de $\left.Y_{S}\right)$. Il est alors clair que les translatés de cet ouvert $U$ par les dites $\begin{array}{ll}\text { quasi-sections recouvrent } G_{S_{1}} & \text { C.Q.F.D. }\end{array}$

3.4.2. Proposition : Soient $S$ un schéma de Dedekind, G un S-schéma en groupes plat et localement de type fini, H un sous-schéma fermé en groupes de G , plat sur $S$, et $G / H$ le S-faisceau fppf quotient. Alors il existe un schéma $S_{1}$ fpqc sur $S$ tel que $(G / H)_{S_{1}}$ soit un $S_{1}$-schéma.

Démonstration : Considérons la relation d'équivalence canonique

$G \times{ }_{S} H \rightrightarrows G$. Puisque le faisceau fppf quotient se construit en se restreignant à des ouverts affines, on se ramène au cas d'une relation d'équivalence plate et de type fini; et 3.1.1 montre alors que $G / H$ est un S-espace algébrique, (loc. PF) séparé et plat sur S. Sachant que G/H est homogène sous G, on termine comme dans 3.4.1. C:Q.F.D. 
Chapitre IV

Applications aux questions de représentabilité

4.0. Les résultats principaux que nous avons en vue sont les suivants.

4.A. Théorème : Soient $\mathbf{S}$ un schéma localement noethérien de dimension $\leqslant 1$, $G$ un S-faisceau fppf en groupes, et $S^{\prime}$ un schéma fpqc sur $S$ tel que $G_{S^{\prime}}$ soit représentable, séparé et localement de présentation finie sur $S^{\prime}$. Alors G est représentable (séparé, localement de type fini) et de type (FA) sur S (i.e., chaque ensemble fini de points de G qui se projette dans un ouvert affine de $S$, est contenu dans un ouvert affine de G; cf. 1.1 .2 (c)).

4.B. Théorème : Soient $S$ un schéma localement noethérien de dimension $\leqslant 1$, et $G$ un $S$-espace algébrique (10c. PF) et séparé en groupes (cf. 1.3.0). Alors G est un schéma.

4.C. Théorème : Soient $\mathrm{S}$ un schéma localement noethérien de dimension $\leqslant 1$, G un S-schéma en groupes localement de type fini, H un sous-schéma fermé en groupes, plat sur $S$. Alors le S-faisceau fppf quotient G/H est un schéma de type (FA) sur $\mathrm{S}$.

Avant d'énoncer un résultat concernant les espaces homogènes (au sens de [18], VI.1.1), donnons une définition.

4.0.1. Définition : Soient $S$ un schéma, G (resp. X) un S-faisceau fppf en groupes (resp. un S-faisceau fppf) localement de présentation finie sur $S$. On suppose que $G$ opère sur $X$. Nous dirons que $X$ est un S-faisceau homogène (fppf) sous G si le morphisme (de faisceaux)

$$
\begin{aligned}
& G \times{ }_{S} X \rightarrow X \times{ }_{S} X \\
& (g, x) \mapsto(g x, x)
\end{aligned}
$$

est couvrant (fppf). Le faisceau $X$ est dit presque homogène (fppf) sous $G$ si $X$ est la réunion d'un nombre fini d'ouverts homogènes (fppf) sous $G$. On dira que $X$ est un S-espace (ou schéma) homogène (resp. presque homogène) sous G, si de 
plus $\mathrm{X}$ est représentable.

4.D. Théorème : Soient $S$ un schéma localement noethérien de dimension $\leqslant 1$, $G$ un S-schéma en groupes de type fini, $X$ un $S$-faisceau ( $f$ ppf) qui est un S-faisceau presque homogène sous $G$, et $S^{\prime}$ un schéma fpqc sur $S$ tel que $X_{S^{\prime}}$ soit représentable, séparé, de présentation finie sur $S^{\prime}$ et couvre $S^{\prime}$. Alors X est un schéma de type (FA) sur $\mathrm{S}$.

\subsection{Quelques remarques préliminaires}

Nous allons nous ramener d'abord au cas $\mathrm{S}$ semi-local, dans les énoncés 4.A 4.D. En effet, il est clair que, si lạ représentabilité est déjà établie, l'assertion "du type (FA) sur S" se démontre en se ramenant au cas $S$ semi-local. D'autre part, pour établir la représentabilité on peut supposer $\mathrm{S}$ local : car, dans 4.A et 4.D il est question de descente fpqc, et c'est là un résultat standard ([17], appendix, lemma 2); pour 4.C on a vu que, dans les conditions de l'énoncé, G/H est un S-espace algébrique (loc. PF), cf. 3.1.1; donc dans les deux cas 4.B et 4.C, il est question de représenter un s-espace algébrique (loc. $\mathrm{PF}$ ); par passage à la limite on peut donc supposer $S$ local.

on peut supposer désormais donc que $S$ est semi-local noethérien de dimension $\leqslant 1$. Rappelons d'abord quelques résultats connus que nous utiliserons dans ce qui suit.

4.1.1. Proposition : Soient $S$ un schéma, $S^{\prime} \rightarrow S$ un morphisme $f$ pqc, $X$ un S-faisceau fpqc tel que $X_{S}$, soit représentable et localement de présentation finie sur $S^{\prime}$. On suppose qu'il existe un morphisme entier et surjectif $T \rightarrow S$ tel que $\mathrm{X}_{\mathrm{T}}$ soit un schéma. Alors, $X$ est représentable sur $S$ si et seulement si chaque fibre de la projection canonique $X_{T} \rightarrow X$ est contenue dans un ouvert affine de $X_{T}$. Voir [21], théorème 4.1 .

4.1.2. Proposition : Soient $\mathrm{S}$ le spectre d'un corps $\mathrm{k}, \mathrm{G}$ un S-schéma en groupes localement de type fini et $H$ un sous-schéma (fermé) en groupes de G. Alors le S-faisceau fppf quotient $G / H$ est un schéma de type (FA). 
Voir, par exemple, la démonstration de SGAD, $\mathrm{VI}_{A}, 3.2$.

4.1.3. Proposition : Soient $\mathrm{T}$ un schéma localement noethérien, $u: T^{\prime} \rightarrow T$ un morphisme de schémas entier et surjectif, dont les fibres sont ensemblistement finies, et $\mathrm{E}$ un ensemble fini de points de $\mathrm{T}$. Alors pour que $\mathrm{E}$ soit contenu dans un ouvert affine de $T$ il faut et il suffit que $u^{-1}(E)=E^{\prime}$ le soit dans $T^{\prime}$. Voir [21], corollaire 3.8 .

Reprenons la situation de 4.A - 4.D et considérons d'abord le cas dim $S=0$, i.e., $S$ semi-local artinien. On peut en fait supposer que $S$ est le spectre d'un corps k (appliquer, par exemple, 4.1 .1 à $4 . \mathrm{A}$ et $4 . \mathrm{D}$, et [7], III, no2, $\S 7$ à $4 . \mathrm{B}$ et 4.C); soit $\overline{\mathrm{k}}$ une clôture algébrique de $\mathrm{k}$. Les théorèmes $4 . \mathrm{A}, 4 . \mathrm{B}$ résultent alors du fait que $G_{k}$ est un schéma de type (FA) (cf:, e.g., [16] I.8.6., et [1] 3.5) ; 4.C n'est rien d'autre que 4.1 .2 ; et pour prouver $4 . D$ sur $k$, on note que, si $G^{\circ}$ est la composante neutre de $G$, alors les composantes connexes (ou irréductibles) de $\mathrm{X}_{\overline{\mathrm{k}}}$ sont de la forme $G_{\overline{\mathrm{k}}}^{0} / \overline{\mathrm{H}}$ pour un sous-groupe (fermé) $\widehat{r}_{\mathrm{de}}^{\bar{H}} \mathrm{G}_{\overline{\mathrm{k}}}^{0}$; et on termine, grâce à 4.1.2, suivant le raisonnement classique de [16] I.8.7.

On supposera désormais donc que $\mathrm{S}$ est semi-local, noethérien, de dimension 1. D'après ce que I'on vient de voir, dans 4.A - 4.D, les fibres ainsi que leurs voisinages infinitésimaux sont représentables (et de type (FA)).

\subsection{Le cas $S$ normal}

Etape 1) L'énoncé du lemme-clé.

4.2.1. Lemme : Soient $S$ un schéma de Dédekind, $X$ un S-faisceau fppf et $S^{\prime}$ un schéma fpqc sur $S$ tel que $X_{S^{\prime}}$ soit représentable et de présentation finie sur $S^{\prime}$. Alors il existe une extension finie $K_{1}$ du corps des fractions $K$ de $S$ telle que, si $S_{1}$ est le normalisé de $S$ dans $K_{1}, X_{S_{1}}$ soit un schéma (de type fini $\left.\operatorname{sur} s_{1}\right)$.

Démonstration : On peut supposer que $S$ est un trait spec $\Lambda$ et que $S^{\prime}$ est affine d'anneau $A^{\prime}$. I'anneau $A^{\prime}$ est limite inductive filtrante de ses 
sous-1-algèbres $\left\{A_{i}\right\}_{i \in I}$ de type fini. Puisque $X_{S^{\prime}}$ est de présentation finie sur $S^{\prime}$, on trouve, par passage à la limite (EGA, IV, 8), un indice $i_{0} \in I$ assez grand tel que $\mathrm{X}_{\mathrm{S}^{\prime}}$ (resp. la donnée de descente $\operatorname{sur} \mathrm{X}_{\mathrm{S}^{\prime}}$ ) provienne d'un schéma $\mathrm{X}_{i}$ (de type fini) sur $\operatorname{Spec} A_{i_{0}}$ (resp. d'une donnée de descente sur $X_{i_{0}}$ ). D'autre part, I'anneau $A^{\prime}$ étant sans 1 -torsion, $A_{i_{0}} I^{\prime e s t ~ e ́ g a l e m e n t, ~ d o n c ~} S_{i_{0}}=S p e c A_{i_{0}}$ est (fidèlement) plat sur $S$. On en conclut que $x_{i_{0}} \leadsto \mathrm{X}_{\mathrm{S}_{i_{0}}}$, i.e., $\mathrm{X}_{\mathrm{S}_{i_{0}}}$ est un schéma (de type fini) sur $\mathrm{S}_{i_{0}}$.

Soit $T$ une quasi-section plate de $S_{i_{0}}=$ Spec $A_{i_{0}}$ sur $S \quad$ (EGA, IV, 17.16.1). Notons $K_{1}$ la plus petite extension quasi-galoisienne de $K$ engendrée par un corps des fractions de ${ }^{T}$ red - Alors ce $K_{1}$ répond à la question. En effet, si $S_{1}$ est le normalisé de $\mathrm{S}$ dans $\mathrm{K}_{1}$, il existe des points de $\mathrm{S}_{1}$ au-dessus des points de $\mathrm{T}_{\text {red }}$, donc il existe un sous-trait ouvert $\mathrm{Z}$ de $\mathrm{S}_{1}$ tel que $\mathrm{X}_{\mathrm{Z}}$ soit un schéma. D'autre part, le groupe de galois $g$ de $K_{1}$ sur $K$ agit comme un groupe de S-automorphismes sur $\mathrm{S}_{1}$; et si $Z^{\prime}$ est un sous-trait ouvert quelconque de $S_{1}$, il existe $\sigma \in$ of tel que $z^{\prime}=(z)^{\sigma}$ (notation évidente). Puisque of laisse invariant "tout ce qui provient de $S$ ", on en conclut que $X_{S_{1}}$ est un schéma.

Etape 2) Réduction au cas des faisceaux plats sur $S$. C.Q.F.D.

En utilisant la notion d'adhérence schématique de la fibre générique d'un S-faisceau X (cf. 1.2.0), la technique du recollement suivant un fermé (cf. 1.2.4 et 1.3.2) et la remarque 1.1 .2 (c) sur la propriété (FA), on se ramène au cas G plat sur S, dans 4.A-4.C. Pour se ramener au cas G, X plats sur S dans 4.D, il suffit de noter que(sur $S$ normal), si $\bar{G}$ (resp. $\bar{X}$ ) est l'adhérence schématique de la fibre générique de G (resp. X), alors $\bar{X}$ est presque homogène sous $\bar{G}$.

Etape 3) $4 . A \Longrightarrow 4 . B$.

En effet, il suffit de rappeler qu'un espace algébrique en groupes (Ioc. PF), plat et séparé sur un schéma de Dédekind $S$, est représentable après un changement de base fpqc $S^{\prime} \rightarrow S$, cf. 3.4.1. 
Etape 4) Démonstration de 4.A - 4.D dans le cas "de présentation finie" $(S$ normal). Remarquons d'abord que dans 4.C, G étant (désormais) plat sur 'S, il existe un schéma $S^{\prime}$ fpqc sur $S$ tel que $(\mathrm{G} / \mathrm{H})_{S^{\prime}}$ est un schéma, cf. 3.4.2. Il s'agit donc, dans les trois cas 4.A, 4.C, 4.D, d'établir une descente fpqc et de prouver la propriété (FA).

On peut supposer que $S$ est un semi-trait. Avec l'hypothèse "de présentation finie", on trouve, d'après 4.2.1., un semi-trait $s_{1}$ entier (plat) et surjectif sur $S$ tel que $G_{S_{1}},(G / H)_{S_{1}}, X_{S_{1}}$ soient des schémas de type fini sur $S_{1}$ - Grâce au critère 4.1 .1 de descente $\mathrm{fpqc}$, et à la proposition 4.1 .3 concernant la propriété (FA), tout revient à prouver le résultat suivant.

4.2.2. Proposition : Soient $S$ un semi-trait, G un S-schéma en groupes plat et de type fini et $X$ un S-espace (ou schéma) presque homogène sous G. Si X est séparé, alors $X$ est de type (FA).

Démonstration : Traitons d'abord le cas où $G$ (et donc $\mathrm{X}$ ) est lisse sur $S$. Dans ce cas, $X$ est $S$-presque homogène également sous la composante neutre $G^{\circ}$ de $G$. D'autre part, considérons les points maximaux des fibres de $X$ sur S; en utilisant [18], VIII.1, ou 3.3.1.1., on trouve un ouvert affine $U$ de $X$ contenant ces points (rappeler que $X$ est séparé); on a donc $G^{\circ} \cdot U=X$. Le résultat suivant montre alors que $X$ est quasi-projectif.

Proposition ([18], v.3.10) : Soient $\mathrm{S}$ un schéma localement noethérien

normal, $G$ un S-schéma en groupes lisse et à fibres connexes, qui opère sur un S-schéma lisse $X$. Soient $U$ un ouvert de $X$ quasi-affine sur $S, X^{\prime} I^{\prime}$ ouvert G.U, et $F_{i}$, $i \in I$, les composantes irréductibles de $X^{\prime}-U$ qui sont de codimension 1. Alors tout cycle $D=\sum_{i \in I} n_{i} F_{i}$, où tous les $n_{i}>0$, définit un diviseur sur $X^{\prime}=G . U$ tel que $\boldsymbol{\&}=\boldsymbol{O}_{X^{\prime}}(D)$ soit S-ample.

Dans le cas où $G, X$ dans 4.2.2 ne sont pas lisses sur $S$, il suffit

(d'après le cas lisse, et 4.1 .3 ) de démontrer le résultat suivant. 
4.2.3. Proposition : Soient $\mathrm{S}, \mathrm{G}, \mathrm{X}$ comme dans 4.2 .2 ( $\mathrm{X}$ non nécessairement séparé). Alors il existe une extension finie $K_{1}$ du corps des fractions $K$ de $S$ telle que, si $S_{1}$ est le normalisé de $S$ dans $K_{1}$, on ait les propriétés suivantes :

i) Le normalisé $\tilde{G}_{1}$ de $\left(G_{S_{1}}\right)$ red est un $S_{1}$-schéma en groupes lisse et de type fini.

ii) Le normalisé $\tilde{\mathrm{x}}_{1}$ de $\left(\mathrm{x}_{\mathrm{S}_{1}}\right)$ red est un $\mathrm{S}_{1}$-espace presque homogène sous la composante neutre $\tilde{G}_{1}^{\circ}$ de $\tilde{G}_{1} ;$ en particulier $\tilde{X}_{1}$ est lisse (et de type fini) $\operatorname{sur} s_{1}$

Démonstration : Il existe une extension finie radicielle $K^{\prime}$ de $K$ telle que, $\left(G_{K^{\prime}}\right)_{\text {red }}$ soit un $K^{\prime}$-groupe lisse $\left(S G A D, V I_{A}\right)$. Si $S^{\prime}$ est le normalisé de $S$ dans $K^{\prime}$, alors $\left(G_{S^{\prime}}\right)_{\text {red }}$ est l'adhérence schématique de $\left(G_{K^{\prime}}\right)_{\text {red }}$ dans $G_{S^{\prime}}$, donc est un $S^{\prime}$-schéma en groupes plat, de type fini et à fibre générique lisse. De même $\left(\mathrm{X}_{\mathrm{S}^{\prime}}\right)_{\text {red }}$ est un $\mathrm{S}^{\prime}$-schéma plat, de type fini et à fibre générique

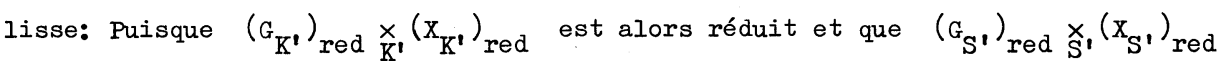
est $S^{\prime}$-plat, on voit que $\left(G_{S^{\prime}}\right)_{\text {red }}$ opère sur $\left(x_{S^{\prime}}\right)_{\text {red }}$ - Quitte à remplacer $S$ par $S^{\prime}, G \operatorname{par}\left(G_{S^{\prime}}\right)_{\text {red }}$ et $X$ par $\left(\mathrm{X}_{S^{\prime}}\right)_{\text {red }}$, on peut supposer donc que $I^{\prime}$ on a une situation du type suivant sur $\mathrm{S}$ :

G (resp. X) est un S-schéma en groupes (resp. un S-schéma) plat, de type fini et à fibre générique lisse ; $G$ opère sur $X$ et chaque fibre $X_{S}$ de $X$ sur $S$ est ensemblistement presque homogène sous $G_{S}$ (ou, ce qui revient au même, sous la composante neutre $G_{S}^{O}$ de $G_{S}$ ).

Appliquons dans ces conditions les corollaires 2, 3 de l'appendice II. Il existe donc une extension finie $K_{1}$ de $K$ telle que, si $S_{1}$ est le normalisé de $S$ dans $\mathrm{K}_{1}$, on ait des propriétés suivantes :

1) Le normalisé $\tilde{G}_{1}$ de $G_{S_{1}}$ est un $S_{1}$-schéma en groupes lisse, de type fini et la projection canonique $\tilde{G}_{1} \rightarrow G_{S_{1}}$ est un $S_{1}$-homomorphisme fini. 
2) Le normalisé $\tilde{X}_{1}$ de $X_{S_{1}}$ est un $S_{1}$-schéma plat, séparable et de type fini.

Puisque, dans ces conditions, $\tilde{G}_{1} \times_{S_{1}} \tilde{X}_{1}$ est normal ([18], IV.2.4), on voit formellement que $\tilde{G}_{1}$ opère sur $\tilde{X}_{1}$ de façon compatible avec l'action de $G_{S_{1}}$ sur $X_{S_{1}} \cdot$ Par ailleurs, les fibres de $\tilde{\mathrm{X}}_{1}$ sur $\mathrm{S}_{1}$ sont équidimensionnelles. Soient $\mathrm{k}$ un corps résiduel de $\mathrm{S}_{1}$ et $\overline{\mathrm{k}}$ sa clôture algébrique; il est clair que les orbites de $\tilde{G}_{1 \bar{k}}^{\circ}$ dans $\tilde{\mathrm{X}}_{1_{\overline{\mathrm{k}}}}$ sont les composantes connexes (ou irréductibles) de $\tilde{\mathrm{X}}_{1_{\overline{\mathrm{k}}}}$. Quitte à remplacer $K_{1}$ par une extension finie (et séparable) de $K_{1}$, on voitrque I'on peut supposer l'assertion ii) de l'énoncé également vérifiée. alors Etape 5) Réduction au cas "de présentation finie" ( $S$ normal).

Notons d'abord que (pour $\mathrm{S}$ normal) il ne reste plus qu'à établir 4.A et 4.C dans le cas où $G$ est plat et localement de présentation finie sur un semi-trait $S$. Nous commençons par établir un lemme.

4.2.4. Lemme : Avec les hypothèses de 4.A supposons de plus que $S$ est un semitrait et que $G$ est plat sur $s$. Soient $s_{1}, \ldots, s_{n}$ les points fermés de $s$ et $z_{i}, 1 \leqslant i \leqslant n$, une composante connexe de $G_{s_{i}}$. Alors, il existe un plus petit sous-foncteur ouvert $G(z),(z)=\left(z_{1}, \ldots, z_{n}\right)$, de $G$ possédant les propriétés suivantes :

i) $\left(G_{(z)}\right)_{s_{i}}=z_{i}, 1 \leqslant i \leqslant n$

ii) la fibre générique $G_{(z)}$ de $G_{(z)}$ est réunion d'un nombre fini de composantes de $G_{\eta}$.

Et par suite :

iii) $\left(G_{(z)}\right)_{S^{\prime}}$ est de présentation finie sur $S^{\prime}$.

Démonstration : Soient $\mathrm{S}_{i}, 1 \leqslant i \leqslant n$, les sous-traits ouverts de $\mathrm{S}$ correspondant aux points fermés $s_{i}$ - Supposons que l'on a construit un ouvert $G_{\left(z_{i}\right)}$ de $G_{i}=G \mid S_{i}$ possédant les propriétés correspondantes à $\left.i\right)$, $\left.i i\right)$, iii) (par rapport à $s_{i}$ et $z_{i}$ ) pour tout $i=1, \ldots, n$. On peut alors poser $G(z)=\bigcup_{i=1}^{n} G_{\left(z_{i}\right)}$ 
On suppose donc que $S=(S, \eta, s)$ est un trait et que $Z$ est une composante connexe de $G_{S}$. On peut aussi supposer que $S^{\prime}$ est le spectre d'un anneau de valuation dominant $\mathrm{S}$; soit $\eta^{\prime}$ (resp. $\mathrm{s}^{\prime}$ ) son point générique (resp. fermé). Notons $z$ le point générique de $z$ et $z_{i}^{\prime}, 1 \leqslant i \leqslant r$, les points maximaux de $z_{s^{\prime}}$. Et posons $G^{\prime}=G_{S^{\prime}}, S^{\prime \prime}=S^{\prime}-\left\{s^{\prime}\right\}$.

Considérons le sous-foncteur ouvert $U$ de $G$ à ensemble sous-jacent $\left(G-\left(G_{S}-Z\right)\right)$. Le sousfoncteur $G_{(Z)}$ de l'énoncé sera obtenu en éliminant de $U$ les composantes de $U_{\eta}=G_{\eta}$ qui ne "générise" pas $Z$. Pour ce faire, remarquons d'abord que, pour tout $i, 1 \leqslant i \leqslant r, \operatorname{spec}\left(\theta_{G^{\prime}, z_{i}^{\prime}}\right) n_{G^{\prime}}^{\prime}$ est affine, donc ne contient qu'un nombre fini de points maximaux de $G_{\eta^{\prime}}^{\prime} ;$ soit donc $Y$ l'ouvert quasicompact de $G_{\eta}$, réunion des composantes de $G_{\eta}$ dont l'image inverse dans $G_{\eta}^{\prime}$ rencontre un des $\operatorname{Spec}\left(O_{G^{\prime}, z_{!}^{\prime}}\right), 1 \leqslant i \leqslant r$. Je dis que $U_{S^{\prime \prime}}-Y_{S^{\prime}}=U_{S^{\prime \prime}}-Y_{S^{\prime \prime}}$ est fermé dans $U_{S^{\prime}} ;$ pour le voir il faut démontrer l'assertion suivante : Si $x^{\prime}$ est un point maximal de $U_{S^{\prime \prime}}-Y_{S^{\prime \prime}}$, alors $\left\{\overline{x^{\prime}}\right\} \cap\left(U_{S^{\prime}}=Z_{S^{\prime}}\right)=\varnothing$.

Mais $U_{S^{\prime}} \hookrightarrow G^{\prime}$ est plat sur $S^{\prime}$ et ses fibres n'ont pas de composantes immergées; donc, si $\left\{\overline{x^{\top}}\right\} \cap z_{s^{\prime}} \neq \varnothing$, chacune des composantes de cette intersection sera de dimension $\operatorname{dim}(\mathrm{z}) \mathrm{F}_{;}$chaque point maximal de $\left\{\overline{x^{1}}\right\} \cap z_{s^{\prime}}$ sera alors un des $z_{i}^{\prime}$, $1 \leqslant i \leqslant r$. Ce qui contredira le choix de $Y$.

Il en résulte $U_{S^{\prime}}-\left(U_{S^{\prime \prime}}-Y_{S^{\prime \prime}}\right)=Y_{S^{\prime}} \cup Z_{S^{\prime}}$ est un ouvert de $U_{S^{\prime}}$ donc de $G_{S^{\prime}}$. Il est alors immédiat que le sous-foncteur $G_{(z)}$ de $G$, à ensemble sous-jacent YUZ, est un ouvert de G satisfaisant aux assertions du lemme. C.Q.F.D.

Nous conservons les hypothèses et les notations de 4.2.4. Le sous-foncteur ouvert de $G$ qui correspond au choix $(\mathrm{z})=\left(\mathrm{G}_{\mathrm{s}_{1}}^{0}, \ldots, \mathrm{G}_{\mathrm{s}_{\mathrm{n}}}^{0}\right.$ ) (où $\mathrm{G}_{\mathrm{s}_{i}}^{0}$ est la composante neutre de $\left.G_{s_{i}}, 1 \leqslant i \leqslant n\right)$ sera noté $G_{(0)}$. Il est formel de voir que c'est un sous-faisceau en groupes de G (c'est le plus petit sous-groupe ouvert de G). Pour un choix de (z) quelconque, il est formel aussi de voir que le morphisme canonique $G_{(0)} \times{ }_{S}^{G}(z) \rightarrow G$ de faisceaux, se factorise par $G(z)$; autrement dit, le S-faisceau en groupes $G(0)$ opère $\operatorname{sur} G_{(z)}$. Puisque, pour tout $s \in S, G_{s} / G_{s}^{0}$ 
est un groupe étale sur $k(s)$ (cf. SGAD, $\mathrm{VI}_{\mathbf{A}}, 5.5$ ), on peut trouver un semi-trait $S_{1}$ fini et étale sur $S$, tel que $G_{(z) S_{1}}$ soit un $S_{1}$-faisceau presque homogène (au sens de 4.0.1) sous $G_{(0) S_{1}}$ (bien entendu $S_{1}$ dépend $d u$ choix de $(z)$ ). Considérons maintenant le cas $4 . \mathrm{C}$ où $\mathrm{G}$ est plat et localement de présentation finie sur $S$ un semi-trait. Considérons le morphisme canonique $G \stackrel{\varphi}{\longrightarrow} G / H$ de faisceaux. Si $G_{(z)}$ est un ouvert de $G$ du type construit plus haut, il est formel de voir que $\varphi\left(G_{(z)}\right)$ est un sous-faisceau ouvert de G/H, car $\varphi$ est couvrant fppf. (Noter aussi que G étant ici un schéma, la construction des $G_{(z)}$ est facile). D'autre part, pour les actions canoniques de G, $\varphi$ est une Grapplication. Donc si $S_{1}\left(=S_{1}((z))\right)$ est tel que $G_{(z) S_{1}}$ soit un $S_{1}$-espace presque homogène sous ${ }^{G}(0) S_{1}$, alors il est clair que $\varphi\left(G_{(z)}\right)_{S_{1}}$ est un $S_{1}$-faisceau presque homogène

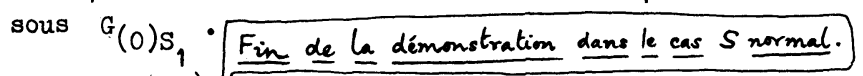

Etape 6). Nous sommes maintenant en mesure de démontrer 4.A et 4.C (cas général) sur S normal ; comme nous l'avons vu, on peut supposer que $S$ est un semi-trait et que $G$ est S-plat. Grâce à 3.4.2., il s'agit, dans les deux cas, de faire une descente fpqc et d'établir la propriété (FA). Nous conservons les notations ci-dessus.

D'après l'étape 4), (où nous avons établi 4.A - 4.D avec I'hypothèse de présentation finie, sur $S$ normal), $G_{(0)}$ est représentable. Pour un (z) quelconque (comme plus haut), il existe un semi-trait $S_{1}$ fini et étale sur $S$ tel que $G_{(z) S_{1}}$

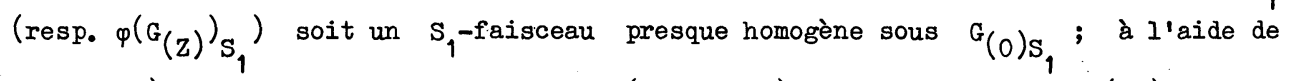
l'étape 4) et la descente finie et plate (classique) d'un schéma de type (FA), on conclut que $G_{(z)}$ (resp. $\varphi\left(G_{(z)}\right)$ ) est représentable. Comme les $G_{(z)}$ (resp. $\left.\varphi\left(G_{(z)}\right)\right)$ recouvrent toutes les fibres spéciales de $G$ (resp. G/H), on a ainsi prouvé la représentabilité de G (resp. G/H). Reste à établir la propriété (FA). Soit $\mathrm{E}$ un ensemble fini de points de $\mathrm{G} / \mathrm{H}$; il suffit d'étudier le cas où chaque point de $E$ est dans une des parties $\varphi\left(G_{(z)}\right)$ de $G / H$; on trouve donc un nombre fini de (z) tel que $E$ soit contenu dans la réunion $X$ de ces $\varphi(G(z)$ ). II existe donc $\mathrm{S}_{2}$ fini et étale sur $\mathrm{S}$ tel que $\mathrm{X}_{\mathrm{S}_{2}}$ soit presque homogène sous 


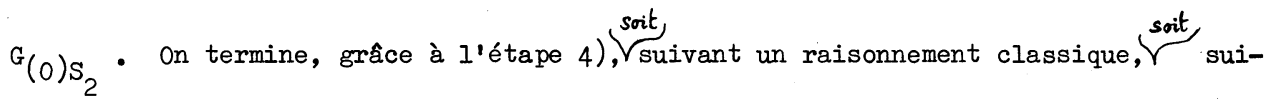
vant 4.1 .3$.

C.Q.F.D.

4.3. Le cas $S$ non-normal; fin de la démonstration.

Comme nous l'avons vu (cf. 4.1) on peut supposer que $\mathrm{S}$ est semi-local de dimension 1. Alors, le normalisé de $S_{\text {red }}$ est somme disjointe de semi-traits et de corps (Krull-Akizuki). Si $\tilde{S}$ est ce normalisé, nous avons établi 4.A-4.D sur $\tilde{S}$ dans 4.2. Il reste donc à faire la descente entière de $\tilde{S}$ à $S$. Pour 4.A et 4.D cela est assurée grâce au critère de descente 4.1 .1 et la propriété (FA) sur $\tilde{S}$. Sachant que dans les deux cas $4 . B$ et $4 . C$ on a affaire à un S-espace algébrique (loc. PF) et séparé (cf. 3.1.1) on termine à l'aide du critère de descente 3.2.1 et la propriété (FA) sur $\tilde{\mathrm{S}}$.

Enfin, la propriété (FA) sur $S$ est assurée par celle sur $\tilde{S}$ et 4.1.3. 


\section{Appendice I}

Passage au quotient par un grouporde plat

Soient $\mathrm{S}$ un schéma quelconque, $\mathrm{R}, \mathrm{X}$ des S-schémas localement de type fini et $R \underset{p_{2}}{\stackrel{p_{1}}{\longrightarrow}} X$ un s-groupoide (cf. SGAD, V, 1) plat et de présentation finie. Nous appellerons graphe de ce groupolde l'image ensembliste du morphisme $\mathrm{d}: \mathrm{R} \stackrel{\left(\mathrm{p}_{1}, \mathrm{p}_{2}\right)}{\longrightarrow} \mathrm{X} \times{ }_{\mathrm{S}} \mathrm{X}$. Nous noterons $\mathrm{X} / \mathrm{R}$ (resp. $\widetilde{X} / \mathrm{R}$ ) l'espace annelé (resp. le S-faisceau fppf) quotient de $X$ par $R$. Pour un morphisme $Y \stackrel{\varphi}{\longrightarrow} X$ dans (Sch/S), nous noterons $R_{Y} \rightarrow Y$ le groupoide induit par $R$ sur $Y$ (cf. SGAD, V, 3). Si $x$ est un point de $X, R_{X} \rightarrow \operatorname{spec} O_{X, X}$ sera le groupoide induit par $R$ sur Spec $O_{X, X}$, pour le monomorphisme canonique $\operatorname{Spec}_{\mathrm{X}, \mathrm{X}} \rightarrow \mathrm{X}$.

Dans cet appendice il est question d'étudier (suivant [19]) des conditions pour que $\mathrm{X} / \mathrm{R}$ (resp. $\widetilde{\mathrm{X} / \mathrm{R}}$ ) soit un S-schéma. Le cas qui nous intéresse surtout est celui où le morphisme $d: R \stackrel{\left(p_{1}, p_{2}\right)}{\longrightarrow} X x_{S} X$ est quasi-fini (e.g., $R$ est une relation d'équivalence sur $\mathrm{X}$ ). (Cette hypothèse restrictive ne sera toutefois faite qu'à partir du corollaire 3).

A - Réduction au cas d'un grouporde quasi-fini

Lemme 1 : Soient $R \underset{p_{2}}{\stackrel{p_{1}}{\longrightarrow}} X$ un groupoide, $Y \stackrel{i}{\longrightarrow} X$ un monomorphisme de schémas, $\mathrm{R}_{\mathrm{Y}} \rightarrow \mathrm{Y}$ le groupoide induit par $\mathrm{R}$ sur $\mathrm{Y}$. On suppose que la restriction de $p_{2}$ à $\mathrm{p}_{1}^{-1}(\mathrm{Y})$ (considéré comme sous-objet de $\mathrm{R}$ ) est un morphisme ouvert couvrant son image pour la topologie fpqc. Alors le morphisme canonique $Y / R_{Y} \stackrel{j}{\longrightarrow} X / R$ est une immersion ouverte d'espaces annelés.

Preuve : Vu la définition du groupoide induit, $j$ est évidemment injectif. L'hypothèse faite entraîne que le R-saturé d'un ouvert de $Y$ est un ouvert de $X$, donc $j$ est une immersion ouverte topologique. Il suffit donc de montrer que si $\operatorname{sat}_{R}(Y)=X$, alors on a $\operatorname{ker}(\Gamma(X) \rightarrow \Gamma(R))=\operatorname{ker}\left(\Gamma(Y) \rightarrow \Gamma\left(R_{Y}\right)\right)$. Or, par hypothèse le R-saturé de $Y$ pour la topologie fpqc est $X$; on en déduit que le faisceau fpqc $\tilde{X}$ quotient de $X$ par $R$ est égal au faisceau fpqc $\tilde{Y}$ quotient de $Y$ par 
$R_{Y} \cdot$ Il suffit alors de remarquer que

$\operatorname{ker}(\Gamma(X) \rightrightarrows \Gamma(R))=\operatorname{Hom}(\tilde{X}, \operatorname{Spec} Z[T])=\operatorname{Hom}(\tilde{Y}, \operatorname{Spec} Z[T])=\operatorname{ker}\left(\Gamma(Y) \rightrightarrows \Gamma\left(R_{Y}\right)\right)$.

Proposition 2 : Reprenons les hypothèses et les notations du début. Soient $\mathbf{s}$ un point quelconque de $S$ et $x$ un point fermé de $x_{s}$. Alors il existe un ouvert $V$ de $X$ saturé sous $R$ et contenant $x$, et un sous-schéma $Z$ de $V$ tel que l'on ait :

(i) $\mathrm{R}_{\mathrm{Z}} \rightarrow \mathrm{Z}$ est plat et de présentation finie

(ii) le morphisme canonique $\widetilde{Z / R_{Z}} \rightarrow \widetilde{v / R_{v}}$ (resp. $z / R_{Z} \rightarrow v / R_{v}$ ) est un isomorphisme

(iii) $z \cap p_{1} p_{2}^{-1}(x)$ (qui est non vide d'après (ii)) est un ensemble fini de $\operatorname{Ens}(x)$.

Si de plus $X$ est localement de présentation finie sur $S$, on peut supposer $\mathrm{Z}$ de présentation finie sur $\mathrm{S}$.

Démonstration : Utilisant le lemme 1, on peut supposer $X$ affine (remplacer $X$ par un ouvert affine contenant $x$ ). Le lemme 7.2 de SGAD, $v$ (où des hypothèses "noethériennes" sur les fibres suffisent) montre qu'il existe un sous-schéma fermé $F$ de $X$, défini par un nombre fini d'équations (donc de présentation finie sur $S$, si $X$ l'est), tel que, si $F_{1}=p_{1}^{-1}(F)$ et si $\bar{p}_{1}, \bar{p}_{2}$ désignent les restrictions de $p_{1}, p_{2}$ à $F_{1}$, on ait :

1) $\bar{p}_{1} \bar{p}_{2}^{-1}(x)$ est fini non vide dans $\operatorname{Ens}(F)$

2) $\bar{p}_{2}$ est plat aux points de $\bar{p}_{2}^{-1}(x)$.

Explicitons alors le grouporde $R$ suivant SGAD, V, page 4. On a ainsi le diagramme

(*)

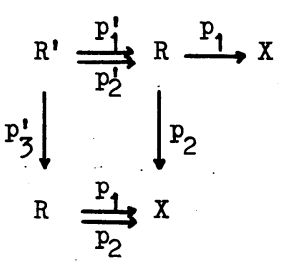

où $R^{\prime}=R\left(p_{1}, p_{2}\right)$, la première suite est exacte et les deux carrés sont cartésiens. 
Posons $\quad F_{2}=\left(p_{1} p_{1}^{\prime}\right)^{-1}(F)=\left(p_{1} p_{2}^{\prime}\right)^{-1}(F) ; \quad c^{\prime}$ est un sous-schéma de $R^{\prime}$; notons avec des lettres soulignées les restrictions aux $F_{i}$ des morphismes de (*). On obtient ainsi le diagramme

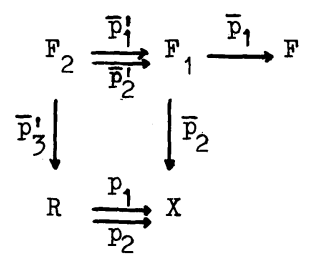

où la première suite est exacte et les deux carrés sont cartésiens. Soit U, le plus grand ouvert de $F_{1}$ au-dessus duquel $\bar{p}_{2}$ est plat; on a donc $U_{1} \cap \bar{p}_{2}^{-1}(x) \neq \varnothing$. D'autre part, comme $p_{1}, p_{2}$ (donc aussi $\bar{p}_{1}^{\prime}, \bar{p}_{2}^{\prime}$ ) sont $f p p f$, on a nécessairement $\left(\bar{p}_{1}^{\prime}\right)^{-1}\left(U_{1}\right)=\left(\bar{p}_{2}^{\prime}\right)^{-1}\left(U_{1}\right)=$ hléplus grand ouvert où $\bar{p}_{3}^{\prime}$ est plat. Puisque la première suite est exacte et que $\bar{p}_{1}$ est ouvert, on voit que $U_{1}=\left(\bar{p}_{1}\right)^{-1}(U)$ pour un ouvert $U$ de $F$; on a évidemment $U n p_{1} p_{2}^{-1}(x) \neq \varnothing$. Or, l'ouvert $U$ de $F$ est la trace sur $F$ d'un ouvert $W$ de $X$; quitte à remplacer $X$ par $W$, on peut donc supposer que $U=F$, donc $U_{1}=F_{1}$, de sorte que $\bar{p}_{2}$ est plat.

Il est alors clair que dans ces conditions $R_{F} \rightrightarrows F$ est plat et de présentation finie : en effet, on a les carrés cartésiens suivants

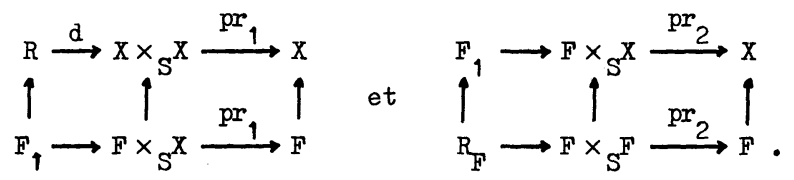

L'ensemble $F \cap p_{1} p_{2}^{-1}(x)$ est fini non vide. D'après le lemme 1 , le morphisme canonique $F / R_{F} \rightarrow X / R$ est une immersion ouverte. Pour établir la proposition 2 il suffit alors de prendre pour $V$ l'ouvert de $X$ égal au R-saturé de $F$ dans $X$. La dernière assertion résulte de la démonstration.

C.Q.F.D.

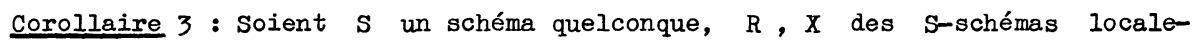
ment de type fini et $R \frac{p_{1}}{p_{2}} X$ un $S$-grouporde plat et de présentation finie tel que le morphisme $\mathrm{d}: \mathrm{R} \stackrel{\mathrm{p}_{2}\left(\mathrm{p}_{1}, \mathrm{p}_{2}\right)}{\longrightarrow} \mathrm{X} \times{ }_{S} \mathrm{X}$ soit quasi-fini. Soient $\mathrm{s}$ un point 
de $S$ et $x$ un point fermé de $X_{S}$. Alors il existe un ouvert $V$ de $X$, saturé sous $\mathrm{R}$ et contenant $\mathrm{x}$, et un sous-schéma $\mathrm{Z}$ de $\mathrm{V}$ tel que l'on ait :

i) $R_{Z} \rightarrow \mathrm{Z}$ est plat, quasi-fini et de présentation finie

ii) le morphisme canonique $\mathrm{z} / \mathrm{R}_{\mathrm{Z}} \rightarrow \mathrm{V} / \mathrm{R}_{\mathrm{V}} \quad\left(\right.$ resp. $\widetilde{\mathrm{Z} / \mathrm{R}_{\mathrm{Z}}} \rightarrow \widetilde{V / \mathrm{R}_{\mathrm{V}}}$ ) est un isomorphisme.

En effet considérons un $\mathrm{V}$ et un $\mathrm{Z}$ donnés par la proposition 2. Si $\mathrm{z}$ est un point de $\mathrm{Z} \cap \mathrm{p}_{1} \mathrm{p}_{2}^{-1}(\mathrm{x})$, alors l'hypothèse faite sur $\mathrm{d}$ et la définition du groupoide induit entrainent que $R_{Z} \rightarrow Z$ est quasi-fini en $z$. En utilisant le "Main Theorem" et en se restreignant à un ouvert convenable de $Z$ on peut supposer que $\mathrm{R}_{\mathrm{Z}} \rightarrow \mathrm{Z}$ est un grouporde plat, quasi-fini et de présentation finie. Il suffit alors de remplacer $V$ par le R-saturé dans $X$ de cet ouvert de $\mathrm{Z}$.

Remarque 4 : Gardons les hypothèses du corollaire 3. Alors on voit que la question de représentabilité de $X / R$ ou de $\widetilde{X} / R$ se ramène au cas où $\frac{R}{p_{2}} X$ est un grouporde quasi-fini, plat et de présentation finie. C'est à ce cas que nous allons nous bormer ci-dessous.

\section{$B$ - Un critère de passage au quotient}

Théorème 5 : Soient $\mathrm{S}$ un schéma quelconque, $\mathrm{R}, \mathrm{X}$ des schémas localement de type fini, $R \underset{p_{2}}{\stackrel{p_{1}}{\longrightarrow}} \times$ un s-grouporde plat, quasi-fini et de présentation finie, $x$ un point de $X,{ }^{2}$ s son image dans $\mathrm{S}$. Alors les conditions suivantes sont équivalentes :

1) Il existe un ouvert $R$-saturé $U$ de $X$ contenant $x$ tel que $U / R_{U}$ soit un schéma.

2) i) Il existe un ouvert $V$ de $X$ contenant $x$ tel que le graphe $\Gamma_{\mathrm{V}}$ de $\mathrm{R}_{\mathrm{V}}$ soit fermé.

ii) Il existe un morphisme $\mathrm{v} \stackrel{\varphi}{\longrightarrow} \mathrm{Z}$ dans (Sch/s) compatible avec

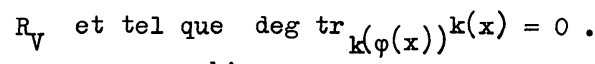

$2^{\text {bis }) ~ i) ~ L e ~ g r a p h e ~} \Gamma_{x}$ du grouporde $R_{x} \rightarrow T=\operatorname{Spec} 0_{X, X}$ est fermé. ii) Il existe un morphisme $\mathrm{T}=\operatorname{Spec} \mathrm{O}_{\mathrm{X}, \mathrm{x}} \stackrel{\varphi}{\longrightarrow} \mathrm{Z}$ dans $(\operatorname{Sch} / \mathrm{s})$ 
compatible avec $R_{x}$ et tel que deg $\operatorname{tr}_{k}(\varphi(x))^{k(x)}=0$.

Enfin, si $X / R$ est un schéma, le morphisme canonique $X \rightarrow X / R$ est universellement ouvert.

La démonstration que nous allons donner prouvera en même temps (vu le corollaire 3) $1 \mathrm{e}$

Théorème 6 : Avec les hypothèses générales du corollaire 3, supposons de plus que $\mathrm{d}: \mathrm{R} \stackrel{\left(p_{1}, p_{2}\right)}{\longrightarrow} \mathrm{X} \times{ }_{S} \mathrm{X}$ est une $\underline{\text { immersion. Alors, }}$ si $X / R$ est un schéma, le morphisme canonique $\widetilde{X / R} \rightarrow X / R$ est un isomorphisme; en particulier, le morphisme canonique $X \rightarrow X / R$ est $f$ ppf.

Démonstration : Il est évident que 1) $\Longrightarrow 2) \Longrightarrow 2^{\text {bis }}$ ). Nous allons montrer que $\left.2^{\text {bis }}\right) \Longrightarrow 1$ ) et en même temps démontrer la dernière assertion du théorème 5 et le théorème 6. Commençons par faire quelques réductions.

a) Réduction au cas où $X$ est affine et $R$ est de graphe fermé. Considérons $\mathrm{T}=\operatorname{Spec} \mathrm{O}_{\mathrm{X}, \mathrm{x}}$ comme limite projective filtrante des voisinages ouverts affines $x_{i}$ de $x$ dans $x$. Soient $R_{i}=R_{X_{i}}, d_{i}: R_{i} \rightarrow x_{i} \times X_{i}$ le mor phisme canonique et $\Gamma_{i}=d_{i}\left(R_{i}\right)=l e$ graphe de $d_{i}$. On a donc l'égalité $\mathrm{T} \times{ }_{\mathrm{S}} \mathrm{T}=\underset{i}{\lim } \mathrm{X}_{i} \times \mathrm{X}_{i}$ et les diagrammes cartésiens suivants :

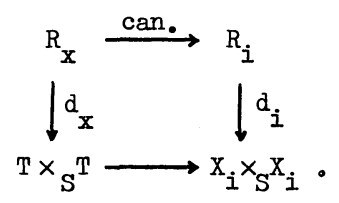

Puisque $d_{i}$ est de présentation finie, $\Gamma_{i}=i m\left(d_{i}\right)$ est une partie constructible de $x_{i} x_{S} X_{i}$ (EGA, IV). D'autre part, $\Gamma_{x}=i m\left(d_{x}\right)$ est fermé par hypothèse dans $\mathrm{T} \times{ }_{S} \mathrm{~T}$. Il résulte de $\mathrm{EGA}, \mathrm{IV} .8$, que $\Gamma_{i}$ est fermé dans $\mathrm{X}_{i} \times_{S} \mathrm{X}_{i}$ pour $i$ assez grand.

b) Réduction au cas $\mathrm{Z}$ de présentation finie. on peut supposer $\mathrm{Z}$ affine. Considérons $\mathrm{Z}$ comme limite projective filtrante de S-schémas affines de présentation finie $\left\{\mathrm{Z}_{\alpha}\right\}$ et soit $\varphi^{(\alpha)}: \mathrm{T} \rightarrow \mathrm{Z} \rightarrow \mathrm{Z}_{\alpha}$ le 
morphisme composé.

Puisque $x_{s}$ est de type fini sur $k(s), \varphi^{(\alpha)}$ a la même fibre que $\varphi$ en $x$ pour $\alpha$ assez grand. Quitte à remplacer $Z$ par $z_{\alpha}$ et $\varphi$ par $\varphi^{(\alpha)}$ pour $\alpha$ assez grand on peut supposer $\mathrm{Z}$ de présentation finie sur $\mathrm{S}$.

c) Réduction au cas où $\varphi$ provient d'un morphisme de $X$ dans $Z$. Puisque $Z$ est de présentation finie sur $S$, le morphisme $\varphi: T \rightarrow Z$ provient d'un morphisme $\varphi_{i}: X_{i} \rightarrow Z$ pour $i$ assez grand (notations de a)). Puisque $R_{x}=\underset{i}{l i m}\left(R_{i}=R_{X_{i}}\right)$ et que $\varphi$ est compatible avec $R_{x}, \varphi_{i}$ est compatible avec $R_{i}$ pour $i$ assez grand. Il suffit alors de remplacer $x$ par un tel $x_{i}$. Grâce au "Main Theorem" on peut aussi supposer $\varphi$ quasi-fini.

Ceci étant, quitte à changer de notations, on peut prendre $S=Z$. On est ainsi ramené à démontrer le

Théorème 7 : Soient $\mathrm{S}$ un schéma quelconque, $\mathrm{X}$ un $\mathrm{S}$-schéma localement quasifini sur $S$ et $R \underset{p_{2}}{\stackrel{p_{1}}{\rightleftharpoons}} X$ un S-groupoide quasi-fini plat et de présentation finie. Alors les conditions suivantes sont équivalentes :

1) $X / R$ est un schéma.

2) Pour tout $x \in X$, il existe un ouvert $U$ de $X$ contenant $x$ tel que le graphe de $R_{U}$ soit fermé. De plus, si $X / R$ est un schéma, le morphisme canonique $X \rightarrow X / R$ est universellement ouvert; enfin si $X / R$ est un schéma et si $\mathrm{d}: \mathrm{R} \stackrel{\left(\mathrm{p}_{1}, \mathrm{p}_{2}\right)}{\longrightarrow} \mathrm{X} \times{ }_{\mathrm{S}} \mathrm{X}$ est une $\underline{\text { immersion }}$ alors le morphisme canonique $\widetilde{X} / \mathrm{R} \rightarrow \mathrm{X} / \mathrm{R}$ est un isomorphisme.

Démonstration : Il s'agit de prouver que 2) $\Longrightarrow 1$ ) et établir les dernières assertions. On peut donc supposer, comme plus haut, que $X$ est affine, quasi-fini sur $S$ et que $R$ est de graphe fermé.

Soit $U$ le plus grand ouvert de $S$ tel que, si $V=$ l'image inverse de $U$ dans $\mathrm{X}$, on ait :

i) $V / R_{V}$ est un schéma quasi-affine sur $U$ et le morphisme canonique $\mathrm{V} \rightarrow \mathrm{V} / \mathrm{R}_{\mathrm{V}}$ est universellement ouvert. 
ii) si d est une immersion, alors le morphisme canonique $\widetilde{V / R_{v}} \rightarrow V / R_{v}$ est un isomorphisme.

On doit prouver que $U=S$, sous I'hypothèse 2). Supposons donc que $U \neq S$ et soit $s$ un point maximal de $S-U$. Alors il existe un voisinage étale $S^{\prime}$ de $s$ sur $S$ tel que l'on ait :

iii) il existe un point unique $s^{\prime} \in S^{\prime}$ au-dessus de $s$

iv) $X^{\prime}=X_{S^{\prime}}$ est somme disjointe $X_{1}^{\prime} \Perp X_{2}^{\prime}$ de deux schémas

v) $X_{1}^{\prime} \leftrightarrow X^{\prime} \rightarrow S^{\prime}$ est fini

vi) $X_{2}^{\prime} \cap X_{s^{\prime}}^{\prime}=\varnothing \quad$ (cf. EGA, IV 18.12).

L'image $S^{\prime \prime}$ de $S^{\prime}$ dans $S$ est un ouvert contenant $s$ et il nous suffit de montrer que $U>S^{\prime \prime}$; on peut donc supposer que $S^{\prime}$ couvre $S$.

Notons $R^{\prime} \stackrel{p_{1}^{\prime}}{\underset{p_{2}^{\prime}}{\longrightarrow}} X^{\prime}$ le $S^{\prime}$-grouporde déduit de $R \underset{p_{2}}{\stackrel{p_{1}}{\longrightarrow}} X$ par le changement de base $S^{\prime} \rightarrow S$. Supposons démontré que $X^{\prime} / R^{\prime}=Y^{\prime}$ est un $S^{\prime}$-schéma quasi-affine vérifiant $i$ ) et $i i)$ ci-dessus sur $S^{\prime}$. Notons d'abord le lemme facile suivant

Lemme 8 : Soient $X^{\prime}$ un schéma, $R^{\prime} \rightrightarrows X^{\prime}$ un grouporde quasi-compact et quasiséparé tel que $Y^{\prime}=X^{\prime} / R^{\prime}$ soit un schéma et tel que le morphisme canonique $X^{\prime} \rightarrow X^{\prime} / R^{\prime}$ soit quasi-compact, quasi-séparé et universellement ouvert, et enfin soit $\mathrm{Y}^{\prime \prime} \rightarrow \mathrm{Y}^{\prime}$ un morphisme plat dans $(\mathrm{Sch})$. Si $\mathrm{R}^{\prime \prime} \rightrightarrows \mathrm{X}^{\prime \prime}$ est le grouporde déduit de $R^{\prime} \rightrightarrows X^{\prime} \quad$ par lẹ changement de base $Y^{\prime \prime} \rightarrow Y^{\prime}$, alors on a $Y^{\prime \prime} \simeq X^{\prime \prime} / R^{\prime \prime}$.

Il en résulte dans notre cas que $Y^{\prime}=X^{\prime} / R^{\prime}$ est muni d'une donnée de descente canonique par rapport au morphisme étale $S^{\prime} \rightarrow S$. Puisque $Y^{\prime}$ est quasi-affine, cette donnée est effective (SGA, 1, VIII) et si Y est le schéma quasi-affine sur $\mathbf{S}$ ainsi obtenu, il est immédiat que $Y$ est égal à $X / R$ et possède les propriétés $i)$, ii) au-dessus de $S$.

On peut donc supposer que $S^{\prime}=S$, et par suite supprimer les "primes" des notations.

Considérons l'ouvert $X_{1}$ de $X$ et le grouporde induit par $R \rightrightarrows X$ sur $X_{1}$, soit $R_{1} \rightarrow X_{1}$. Comme $R$ est de graphe fermé, il en est de même $R_{1}$. Dans le cas 
où $R \stackrel{d}{\longrightarrow} X x_{S} X$ est une immersion, cela entraine que $R_{1} \stackrel{\text { can. }}{\longrightarrow} X_{1} \times_{S} X_{1}$ est une immersion fermée, de sorte que $R_{1} \rightarrow X_{1}$ est une relation d'équivalence finie et plate ( $\operatorname{car} \mathrm{X}_{1} \rightarrow \mathrm{S}$ est $\underline{\text { fini }}$ ). Donc si $\mathrm{X}_{1} / \mathrm{R}_{1}$ est un schéma, alors $\widetilde{\mathrm{X}_{1} / \mathrm{R}_{1}} \sim \mathrm{x}_{1} / \mathrm{R}_{1}$ (SGAD, V, 4.1). Dans le cas général, l'image schématique de $R_{1}$ dans $X_{1} \times_{S} X_{1}$ par le morphisme canonique est un sous-schéma fermé $\Gamma_{1}$ de $x_{1} x_{S} x_{1}$; et les projections canoniques $\Gamma_{1} \Rightarrow X_{1}$ définissent sur Ens $\left(X_{1}\right)$ la même relation d'équivalence que $\mathrm{R}_{1}$ - On a alors le

Lemme 9 : Soient $X, R$ deux S-schémas entiers et supposons que les fibres de $\mathrm{X} / \mathrm{S}$ ont un nombre fini de points. Alors pour toute S-double flèche $\mathrm{R} \rightarrow \mathrm{X}$ qui définit sur $\operatorname{Ens}(X)$ une relation d'équivalence, le conoyau dans la catégorie des espaces annelés est un schéma $Y$, entier sur $S$. Et le morphisme canonique $X \rightarrow Y$ est entier.

Preuve : On peut supposer $S$ donc $R, X$ affines. Si $Y=\operatorname{Spec}(\operatorname{ker}(\Gamma(X) \rightrightarrows \Gamma(R)))$ il est évident que $Y$ est entier sur $S$, et que le morphisme canonique $X \rightarrow Y$ est entier, dominant, donc surjectif. Il faut voir que $Y$ a le bon espace sous-jacent. Quitte à remplacer $S$ par $Y$, on peut supposer que $\Gamma(S)=\operatorname{ker}(\Gamma(X) \rightrightarrows \Gamma(R))$, hypothèse qui se conserve par extension plate. Soit $s \in S$ et montrons que $X_{s}$ est une seule classe d'équivalence. Remplaçons $\mathrm{S}$ par le hensélisé de $\mathrm{O}_{\mathrm{S}, \mathrm{s}}$; X est alors somme d'un nombre fini de composantes locales. Si $X_{S}$ contient plus d'une orbite, la double flèche $R \rightrightarrows X$ se décomposera en somme de deux double flèches

$$
\begin{aligned}
& \mathrm{R}_{1} \Rightarrow \mathrm{x}_{1} \\
& \Perp \Perp ⿻ 上 丨 \\
& \mathrm{R}_{2} \Rightarrow \mathrm{x}_{2} \text {; }
\end{aligned}
$$

il existera donc dans $\Gamma(S)=\operatorname{ker}(\Gamma(x) \rightrightarrows \Gamma(R))$ un idempotent non-trivial, ce qui contredit la connexité de $\mathrm{S}$. Z.Q.F.D.

Il résulte de ce lemme que le conoyau de la double flèche $\Gamma_{1} \Rightarrow X_{1}$ est un schéma $Y_{1}$ - Dans le cas où d est une immersion, on a vu que c'est aussi le quotient faisceautique $\widetilde{\mathrm{x}_{1} / \Gamma_{1}}=\widetilde{\mathrm{X}_{1} / \mathrm{R}_{1}}$. Dans le cas général on a 
Lemme 10 : Le morphisme canonique $x_{1} / R_{1} \stackrel{i}{\longrightarrow} Y_{1}$ est un isomorphisme; et le morphisme canonique $\mathrm{X}_{1} \rightarrow \mathrm{Y}_{1}$ est universellement ouvert.

Preuve : On a vu plus haut que $\Gamma_{1}$ et $R_{1}$ définissent la même relation d'équivalence sur Ens $\left(x_{1}\right)$; donc $i$ est un homéomorphisme topologique. Pour voir que $i$ est un isomorphisme d'espaces annelés, il suffit de voir (quitte à remplacer $x_{1}$ par l'image inverse d'un ouvert affine quelconque de $\left.Y_{1}\right)$ que $\operatorname{ker}\left(\Gamma\left(x_{1}\right) \rightarrow \Gamma\left(R_{1}\right)\right)=$ $=\operatorname{ker}\left(\Gamma\left(\mathrm{x}_{1}\right) \rightarrow \Gamma\left(\Gamma_{1}\right)\right) ;$ mais puisque $\Gamma_{1}$ est l'image schématique de $\mathrm{R}_{1}$, l'application canonique $\Gamma\left(\Gamma_{1}\right) \rightarrow \Gamma\left(R_{1}\right)$ est injective, d! où l'assertion.

Soit alors $r: X_{1} \rightarrow Y_{1}$ le morphisme canonique; d'après le lemme 9 , $r$ est entier, surjectif. Nous allons montrer que $r$ est universellement ouvert. Soit donc $\mathrm{T} \in(\mathrm{Sch} / \mathrm{S})$ et $\mathrm{R}_{1_{\mathrm{T}}} \rightarrow \mathrm{X}_{1_{\mathrm{T}}} \stackrel{\mathrm{r}_{\mathrm{T}}}{\longrightarrow} \mathrm{Y}_{1_{\mathrm{T}}}$ le résultat du changement de base $\mathrm{T} \rightarrow \mathrm{S}$. Puisque le morphisme canonique $R_{1} \rightarrow X_{1} X_{Y_{1}} X_{1}$ est surjectif, il en est de même de $\mathrm{R}_{1_{\mathrm{T}}} \rightarrow \mathrm{X}_{1_{\mathrm{T}}} \mathrm{X}_{\mathrm{T}_{\mathrm{T}}} \mathrm{X}_{1_{\mathrm{T}}}$; il s'ensuit que si $\mathrm{M}$ est un ouvert de $\mathrm{X}_{1_{\mathrm{T}}}, \mathrm{N}=\left(\mathrm{r}_{\mathrm{T}}\right)^{-1}\left(\mathrm{r}_{\mathrm{T}}(\mathrm{M})\right)$ est aussi ouvert, puisque c'est le saturé de $M$ sous $R_{1_{T}}$ et que $R \rightarrow X$ (étant fppf) est universellement ouvert. Mais alors $r_{T}(M)=r_{T}(N)$ est ouvert dans $Y_{1_{T}}$ puisque $r_{\mathrm{T}}$ est fermé surjectif.

C.Q.F.D.

On est maintenant en mesure de prouver que $Y=X / R$ est un schéma vérifiant i), ii) plus haut. Soit $W$ le schéma $V / R_{V}$, quasi-affine sur $U$. Notons $\bar{Y}_{1}$ (resp. $\bar{W}$ ) I'image de $X_{1} \cap V=\bar{X}_{1}$ dans $Y_{1}$ (resp. W). D'après le lemme 1 , $\overline{Y_{1}}$ et $\bar{W}$ sont des ouverts, s'identifiant canoniquement au quotient de $\overline{X_{1}}$ par le groupoide induit de $\mathrm{R}$; et dans le cas où $\mathrm{d}$ est une immersion, ce sera aussi le quotient faisceautique. Par recollement on obtient donc un schéma $Y$ qui est un quotient $X / R$ qui satisfait aux assertions du théorème 7. Reste à voir que $Y$ est quasi-affine; comme $W$ est quasi-affine sur $U$, donc séparé et que $X_{1} \cap V=\bar{X}_{1}$ est fini sur $U$, I'image $\bar{W}$ de $\overline{X_{1}}$ dans $W$ est fermée et ouverte de sorte que $Y$ est somme disjointe de $Y_{1}$ et un ouvert $Y_{2}$ (le complémentaire de $\bar{W}$ dans $W$ ). Puisque $W$ est U-quasi-affine, $Y_{2}$ est quasi-affine (sur $S$ ); enfin $Y_{1}$ est entier donc affine sur $\mathrm{S}$ (lemme 9); d'où le fait que $Y$ est quasi-affine. 
Remarque 11 : Supposons que $X / R$ est un schéma. Alors la même démonstration prouve que le morphisme canonique $X \rightarrow X / R$ est de type fini et que si $S$ est localement noethérien, alors $X / R$ est aussi localement de type fini sur $S$. 


\section{Appendice II}

Normalisé d'un schéma sur un schéma de Dedekind

On se propose dans cet appendice de démontrer le résultat suivant de Raynaud. Théorème 1 : Soient $S$ un schéma de Dedekind ( $c f .1 .2 .0$ ), $\mathrm{K}$ son corps des fractions, $\overline{\mathrm{K}}$ une clóture algébrique de $\mathrm{K}$, et $\overline{\mathrm{S}}$ le normalisé de $\mathrm{S}$ dans $\overline{\mathrm{K}}$. Supposons que $X$ est un S-schéma plat et localement de type fini tel que $X_{K}$ soit géométriquement normal. Alors le normalisé $\widetilde{X}_{\bar{S}}$ de $X_{\bar{S}}$ est un schéma plat, séparable, localement de présentation finie sur $\bar{S}$, et fini sur $X_{\bar{S}}$.

Avant de procéder à la démonstration, donnons quelques corollaires.

Corollaire 2 : Soient $\mathrm{S}, \mathrm{K}, \overline{\mathrm{K}}, \overline{\mathrm{S}}, \mathrm{X}$ comme ci-dessus et supposons de plus que $X$ est de type fini sur $S$. Alors il existe une extension finie $K_{1}$ de $K$ telle que, si $\mathrm{S}_{1}$ est le normalisé de $\mathrm{S}$ dans $\mathrm{K}_{1}$, alors le normalisé $\tilde{\mathrm{X}}_{\mathrm{S}_{1}}$ de $\mathrm{X}_{\mathrm{S}_{1}}$ soit plat, séparable, de type fini sur $\mathrm{S}_{1}$ et fini sur $\mathrm{X}_{\mathrm{S}_{1}}$.

En effet, puisque la propriété pour une fibre d'être séparable est constructible, le corollaire résulte du théorème 1 par passage à la limite (EGA, IV, 8) et le critère de normalité de Serre ([18], IV, 2.4).

Corollaire 3 : Avec les hypothèses du corollaire 2, supposons de plus que X est un S-schéma en groupes. Si $K_{1}, S_{1}$ sont comme dans le corollaire 2, alors le normalisé $\tilde{\mathrm{X}}_{\mathrm{S}_{1}}$ de $\mathrm{X}_{\mathrm{S}_{1}}$ est un $\mathrm{S}_{1}$-schéma en groupes lisse et la projection canonique $\tilde{\mathrm{X}}_{\mathrm{S}_{1}} \rightarrow \mathrm{X}_{\mathrm{S}_{1}}$ est un $\mathrm{S}_{1}$-homomorphisme.

En effet, sous les hypothèses du corollaire $2, \tilde{\mathrm{X}}_{\mathrm{S}_{1}} \times_{\mathrm{S}_{1}} \tilde{\mathrm{X}}_{\mathrm{S}_{1}}$ est normal ([18], IV, 2.4), et le corollaire 3 résulte donc formellement du corollaire 2.

Démonstration du théorème 1 : Modulo passage à la limite, il est clair que le théorème 1 résulte du théorème plus général suivant.

Théorème $1^{\prime}$ : Soient $\Lambda$ un anneau de valuation de hauteur 1 , dont le corps des fractions $K$ est algébriquement clos, $S=\operatorname{Spec} \Lambda$, $t$ le point générique de $S$, s son point fermé, $X$ un S-schéma plat, localement de type fini et à fibre géné- 
rique normale. Alors, le normalisé $\tilde{X}$ de $X$ est plat, séparable, localement de présentation finie sur $S$, et fini sur $X$.

Démonstration : On peut supposer $X$ affine intègre, d'anneau $A$. Si $\operatorname{dim} \mathrm{X}_{\mathrm{K}}=\mathrm{n}$, alors $\mathrm{X}_{\mathrm{S}}$ a toutes ses composantes irréductibles de dimension $\mathrm{n}$. D'après le lemme de normalisation de Noether, il existe un S-morphisme $\varphi: X \rightarrow S\left[T_{1}, \ldots, T_{n}\right]$ tel que $\varphi_{S}$ soit fini (et surjectif si $X_{s} \neq \varnothing$ ). On en conclut qu'il existe un diagramme commutatif

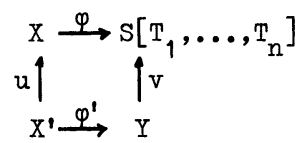

où $u, v$ sont étales affines, $\varphi^{\prime}$ est fini, $u\left(X^{\prime}\right) \supset X_{S}$ et $v_{S}: Y_{S} \cong S\left[T_{1}, \ldots, T_{n}\right]_{S}$, (cf. [22], ch.XI, page 119). Il suffit de prouver le théorème pour $X^{\prime}$. On suppose donc que $X$ est fini sur $Y$.

Soient A I'anneau de $Y$ et B (resp. C) celui X (resp. du normalisé $\tilde{X}$ ). Montrons d'abord que $\mathrm{C}$ est fini sur $\mathbf{A}$. Soient $\Lambda^{\prime}$ le complété de $\Lambda$, et $\mathbf{A}^{\prime}$, $B^{\prime}$ les complétés de A,B pour la topologie définie par élément $\lambda$ de $\Lambda$ de valuation $>0$. Alors $B^{\prime}$ est fini sur $A^{\prime}$. Soient $\eta$ un point maximal de $Y_{s}$ et $\eta^{\prime}$ le point correspondant de $\operatorname{Spec} A^{\prime}$. Puisque $A^{\prime}$ est isomorphe à l'anneau de séries formelles restreintes en les $n$-variables $\mathrm{T}_{1}, \ldots, \mathrm{T}_{\mathrm{n}}$ sur $\Lambda$, il est clair que $\mathbf{A}_{\eta}^{\prime}$, est un anneau de valuation dominant l'anneau de valuation $\mathbf{A}_{\eta}$ (et $\mathbf{A}_{\eta}$ est dense dans $\left.A_{\eta}^{\prime}\right)$.

Par ailleurs, comme $X_{K}$ est normal et $K$ algébriquement clos, si $K$ ' est 'le corps des fractions de $\Lambda^{\prime}, B_{K^{\prime}}$ est normal; par les propriétés d'excellence de la géométrie rigide sur $K^{\prime}(\mathrm{cf} .[12])$ on conclut que $B_{K^{\prime}}^{\prime}$ est normal. Enfin, soit D' la clôture intégrale de $A^{\prime}$ dans $B_{K^{\prime}}^{\prime} ;$ d'après Grauert-Remmert (cf. [9]), $D^{\prime}$ est $\underline{\text { fini }}$ sur $A^{\prime}$.

Or, pour montrer que $\mathrm{C}$ est fini sur $\mathbf{A}$, il suffit de montrer que le morphisme canonique $C \otimes_{A^{A}} A^{\prime} D^{\prime}$ est un isomorphisme : en effet, le problème se situe 
aux points de $(\operatorname{Spec} A)_{S}$ et il suffit alors de remarquer que Spec A' $\rightarrow$ Spec A est

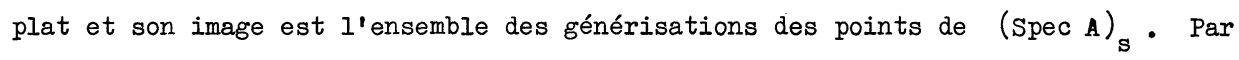
ailleurs, si $Z$ est la partie de $Y_{s}$ complémentaire des points maximaux, il résulte des propriétés de profondeur que $\mathrm{C}$ et $\mathrm{D}^{\prime}$ sont Z-clos (cf. EGA, IV, 5.10). II suffit donc de prouver que $C \otimes_{A^{A}}{ }^{\prime} \rightarrow D^{\prime}$ est un isomorphisme après localisation en chaque point maximal de $A^{\prime} / \lambda^{\prime}$. Ce dernier point est une conséquence du

Lemme $2^{\prime}$ : Soient $\Lambda$ un anneau de valuation, $K$ son corps des fractions, I une extension finie de $K, \bar{\Lambda}$ le normalisé de $\Lambda$ dans $L, \Lambda^{\prime}$ un anneau de valuation dominant $\Lambda$ tel que $\Lambda$ soit dense dans $\Lambda^{\prime}, K^{\prime}$ son corps des fractions, $L^{\prime}=K^{\prime} \otimes_{K} I$, et $\overline{\Lambda^{\prime}}$ la clôture intégrale de $\Lambda^{\prime}$ dans $L_{\text {red }}^{\prime} \cdot$ Alors le morphisme canonique $\bar{\Lambda} \otimes_{\Lambda^{\prime}} \rightarrow \overline{\Lambda^{\prime}}$ est surjectif et son noyau est le nil-radical de $\bar{\Lambda} \otimes_{\Lambda^{\prime}}$. Par conséquent, si $L^{\prime}$ est réduit on a $\bar{\Lambda} \otimes \Lambda_{\Lambda^{\prime}} \cong \bar{\Lambda}^{\prime} ;$ si de plus $\overline{\Lambda^{\prime}}$ est fini sur $\Lambda^{\prime}$, alors $\bar{\Lambda}$ est fini $\operatorname{sur} \Lambda$.

Preuve : Notons que $L_{\text {red }}^{\prime}$ est un produit fini de corps $L_{i}^{\prime \prime}$ extensions finies de $K^{\prime}$. Par suite, $\overline{\Lambda^{\prime}}$ est le produit des normalisés $\overline{\Lambda_{i}^{\top}}$ de $\Lambda^{\prime}$ dans $L_{i}^{\prime \prime}$. On conclut donc (suivant Bourbaki [4], ch.VI, $\S 8, \mathrm{n} 01$, et remarque au théorème 1) que $\bar{\Lambda}$ (resp. $\overline{\Lambda^{\top}}$ ) est un anneau semi-local, dont les composants locaux sont des anneaux de valuation, de groupe de valuation contenant celui de $\Lambda$ (resp. $\Lambda^{\prime}$ ) comme un sous-groupe d'indice fini. Notons $\Gamma$ le groupe associé à $\Lambda$; puisque $\Lambda$ est dense dans $\Lambda^{\prime}, \Gamma$ est aussi le groupe associé à $\Lambda^{\prime}$. Soient $\bar{\Lambda}_{i}, i=1,2, \ldots, s$ les composants locaux de $\bar{\Lambda}$ et $\Gamma_{i}$ le groupe associé à $\bar{\Lambda}_{i}, 1 \leqslant i \leqslant s$. Soit b' un élément de $\overline{\Lambda^{\prime}}$; alors $b^{\prime}$ est l'image d'un $l^{\prime} \in I^{\prime}$ de la forme $\sum_{\alpha=1}^{n} a_{\alpha}^{\prime} \otimes b_{\alpha}, a_{\alpha}^{\prime} \in K^{\prime}, l_{\alpha} \in I$. Puisque $\Gamma$ est d'indice fini dans $\Gamma_{i}$, $1 \leqslant i \leqslant s$, on peut trouver, pour chaque $\alpha=1,2, \ldots, n$, un $r_{\alpha} \in \Gamma$ tel que $r_{\alpha}+v_{i}\left(l_{\alpha}\right)>0$ (où $v_{i}$ est la valuation correspondante à $\bar{\Lambda}_{i}$ ), pour $1 \leqslant i \leqslant s$. Mais $\Lambda$ est dense dans $\Lambda^{\prime}$, donc $K$ l'est dans $K^{\prime}$, et pour tout $\alpha$, il existe un $a_{\alpha} \in K$ tel que, si $a_{\alpha}^{\prime \prime}=a_{\alpha}^{\prime}-a_{\alpha}$, alors $v\left(a_{\alpha}^{\prime \prime}\right) \geqslant r_{\alpha}$ (où $v$ est la valuation correspondante à $\Lambda^{\prime}$ ). On a donc $\sum a_{\alpha}^{\prime} \otimes \ell_{\alpha}=\Sigma a_{\alpha} \otimes \ell_{\alpha}+\sum a_{\alpha}^{\prime \prime} \otimes \ell_{\alpha} \cdot$ Soit b un 
élément de $\mathrm{K}$ ayant la même valuation que $a_{\alpha}^{\prime \prime}, 1 \leqslant \alpha \leqslant n$. Alors $b_{\alpha} \otimes l_{\alpha}=b_{\alpha} \cdot l_{\alpha}$ a une valuation positive dans chacun des $\Gamma_{i}, 1 \leqslant i \leqslant s$, donc est dans $\bar{\Lambda} ;$ par suite, $\quad a_{\alpha}^{\prime \prime} \otimes l_{\alpha}=\left(a_{\alpha}^{\prime \prime} / b_{\alpha}\right)\left(b_{\alpha} \otimes l_{\alpha}\right)$ est dans $\bar{\Lambda} \otimes_{\Lambda^{\prime}}$. On en déduit que l'image dans $L_{\text {red }}^{\prime}$ de $\Sigma a_{\alpha} \otimes l_{\alpha} \in I$ est dans $\overline{\Lambda^{\top}}$, donc $\Sigma a_{\alpha} \otimes l_{\alpha}$ est un élément de $L^{\prime}$ entier sur $\Lambda^{\prime}$. Par descente plate, $\Sigma a_{\alpha} \otimes \ell_{\alpha} \in \bar{\Lambda} ;$ d'où le fait que b' est dans l'image de $\bar{\Lambda} \otimes_{\Lambda} \Lambda^{\prime}$. C.Q.F.D.

L'assertion du théorème concernant la présentation finie résulte du Lemme $3^{\prime}$ : Soient $S$ un schéma intègre, $X$ un S-schéma de type fini et $x$ un point de $X$ où $X$ est plat sur $S$. Alors il existe un voisinage ouvert de $X$, plat et de présentation finie sur $\mathrm{S}$.

Voir [11], corollaire (3.4.7).

Reste à prouver que $\tilde{\mathrm{x}}_{\mathrm{s}}$ est séparable, i.e., que $\tilde{\mathrm{x}}_{\mathrm{s}}$ est réduit, puisque $k(s)$ est algébriquement clos. Or, avec les notations introduites plus haut, $\tilde{\mathrm{X}}=$ Spec $\mathrm{C}$ est Z-clos, et pour voir que $\tilde{\mathrm{X}}_{\mathrm{s}}$ est réduit, il suffit donc de voir qu'il en est ainsi aux points maximaux.

Soit $\eta$ un point maximal de $Y_{S}=(\operatorname{Spec} A)_{S} ;$ alors $A_{\eta}$ est un anneau de valuation; et l'anneau semi-local $C_{\eta}$ est le normalisé de $A_{\eta}$ dans le corps des fractions $F(C)$ de $C$, extension finie du corps des fractions $F(A)$ de $A$. On en conclut que les composants locaux $C_{i}, 1 \leqslant i \leqslant r$, de $C_{\eta}$ sont des anneaux de valuation, dominant $A_{\eta}$ et à groupes $\Gamma_{i}, 1 \leqslant i \leqslant r$, ayant $\Gamma$, celui de $A_{\eta}$, comme sous-groupe d'indice fini. Mais $K$ étant algébriquement clos, le groupe de valuation $\Gamma$ de $A$ est divisible, donc $\Gamma=\Gamma_{i}, 1<i<r$. Remarquons par ailleurs que, par un choix convenable (et nouveau) de $Y$, on peut supposer aussi que les extensions résiduelles de $C_{\eta}$ sur $A_{\eta}$ sont triviales. On a donc que $A_{\eta}$ est dense dans $C_{i}$, pour tout $i$.

D'autre part, soient $\hat{F(A)}$ (resp. $F \hat{(C)})_{i}$ ) le complété de $F(A)$ (resp. $F(C)$ ) pour la valuation associé à $A_{\eta}$ (resp. $\left.C_{i}, 1 \leqslant i \leqslant r\right)$. Puisque $K$ est algébriquement clos, $F(A)$ et $F(C)$ sont des extensions séparables de $K$, et on a donc 


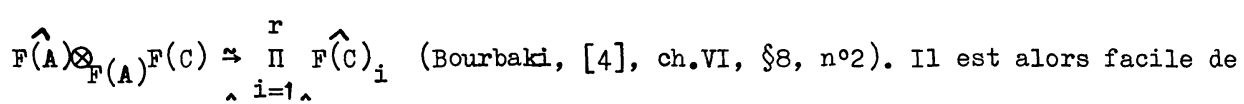
voir que, si $\hat{A}_{\eta}, \hat{C}_{i}$ sont les complétés des anneaux de valuation $A_{\eta}, C_{i}$, $1 \leqslant i \leqslant r$, on a l'égalité $C \otimes_{A_{\eta}} \hat{A}_{\eta} \cong \prod_{i=1}^{r} \hat{C}_{i}$ (raisonnons, par exemple, comme dans le lemme $2^{\prime}$ ).

Puisque, sous les hypothèses faites, on a $\hat{A}_{\eta} \cong \hat{C}_{i}$ pour tout $i$, cela entraîne que $C$ est étale sur A aux points maximaux. On termine par remarquer que $\mathrm{Y}_{\mathrm{s}}$ est réduit. C.Q.F.D.

Remarque : Soient $S$ un semi-trait et $X$ un schéma plat et localement de type fini sur $S$ et à fibre générique lisse. Si le normalisé $\tilde{X}$ de $X$ est lisse, alors il est immédiat que $\tilde{X}$ est la lissification (N) de $X$ sur $S$ (voir ch.II). 
S. ANANTHARAMAN

\section{Bibliographie}

[1] ARTIN (M.). - The implicit function theorem in algebraic geometry, Algebraic geometry, Papers presented at the Bombay Colloquium, 1968 ; p. 13-34. Bombay, Tata Institute for fundamental Research ; London, Oxford University Press, 1969 (Tata Institute. Studies in Mathematics, 4).

[2] ARTIN (M०). - Algebraization of formal moduli, I., Global analysis, Papers in honour of K. Kodaira ; p. 21-71. - Princeton, Princeton University Press and University of Tokyo Press, 1969 (Princeton mathematical Series, 29).

[3] ARTIN (M.). - Algebraic approximation of structures over complete local rings. - Paris, Presses universitaires de France, 1969 (Institut des Hautes Etudes Scientifiques. Publications mathématiques, 36 ; p. 23-58).

[4] BOURBAKI (N.). - Algèbre commutative. Chapitres 5-6. - Paris, Hermann, 1964 (Act. scient. et ind., 1308 ; Bourbaki, 30).

[5] DIEUDONNE ( $\mathrm{J}_{\bullet}$ ) et GROTHENDIECK (A.). - Eléments de géonétrie algébrique (EGA). - Paris, Presses universitaires de France, 1960 ... (Institut des Hautes Etudes Scientifiques. Publications mathématiques, no $4 \ldots$...).

[6] DEMAZURE (M.) et GROTHENDIECK ( $\mathrm{A}_{\bullet}$ ). - Schémas en groupes (SGAD), Séminaire à I' I. H. E. S., exposés V à XVII.

[7] DEMAZURE (M.) et GABRIEL (P.). - Groupes algébriques. Tome 1. - Amsterdam, North-Holland publishing Company, 1970.

[8] GIRAUD (J.). - Méthode de la descente, Bull. Soc. math. France, Mémoire 2, 1964, VIII + 150 p.

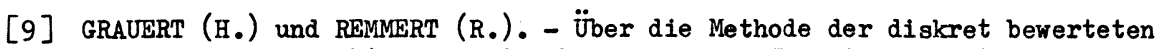
Ringe in der nicht-archimedischen Analysis, Inventiones Math., Berlin, $t .2$, 1966, p. 87-133.

[10] GROTHENDIECK (A.). - Séminaire de géométrie algébrique (SGA), I. H. E. S., 1960-1962, exposés I à VIII.

[11] GRUSON (L.) et RAMIUD (M.). - Critòres de platitude et de projectivité ...., Inventiones Math., Berlin, t. 13, 1971, p. 1-89.

[12] KIEHL ( $R_{0}$ ). - Ausgezeichnete Ringe in der nicht-archimedischen analytischen Geometrie, Math. Inst. der Univ. Minster (Notes privées).

[13] KNUTSON (D.). - Algebraic spaces. - Berlin, Springer-Verlag, 1971 (Lecture Notes in Mathematics, 203).

[14] LANG (S.). - Abelian varieties. - New York, Interscience Publishers, 1959 (Interscience Tracts in pure and applied Mathematics, 7).

[15] MUMFORD (D.). - Abelian varieties. - Bombay, Tata Institute for fundamental Research ; London, 0xford University Press, 1970 (Tata Institute. Studies in Mathematics, 5).

[16] MURRE (J. P.). - On contravariant functors from the category of preschemes over a field into the category of abelian groups. - Paris, Presses universitaires de France, 1964 (Institut des Hautes Etudes Scientifiques. Publications mathématiques, 23 ; p. 5-43).

[17] MURRE (J. P.). - Representation of unramified functors, Applications, Séminaire Bourbaki, 17e année, 1964/65, n० 294, 29 p.

[18] RAYNAUD (M.). - Faisceaux amples sur les schémas en groupes et les espaces homogènes. - Berlin, Springer-Verlag, 1970 (Lecture Notes in Mathematics, 119).

[19] RAYNAUD (M०). - Sur le passage au quotient par un grouporde plat, C. R. Acad. Sc. Paris, t. 265, 1967, Série A, p. 384-387. 
[20] RaYNaUd (M.). - Modèles de Néron, C. R. Acad. Sc. Paris, t. 262, 1966, Série A, p. 345-347.

[21] RAYNAUD (M.). - Un critère d'effectivité de descente, Séminaire Samuel : Algèbre commutative, 1967/68 : Les épimorphismes d'anneaux, $n^{\circ} 5,22 \mathrm{p}$.

[22] RAYNAUD (M.) . - Anneaux locaux henséliens. - Berlin, Springer-Verlag, 1970 (Lecture Notes in Mathematics, 169).

[23] ROSENLICHT (M.). - Some basic theorems on algebraic groups, Amer. J. of Math., t. 78, 1956, p. 401-443.

[24] Séminaire Heidelberg-Strasbourg : Groupes algébriques linéaires, 1965/66. Strasbourg, Publications de I' I. R. M. A., 1967.

Sivaramakrishna ANANTHARAMAN

24 rue Berbier du Mets

75013 PARIS 